\title{
COST-SHARING CONTRACT IN A CLOSED-LOOP SUPPLY CHAIN CONSIDERING CARBON ABATEMENT, QUALITY IMPROVEMENT EFFORT, AND PRICING STRATEGY
}

\author{
Ata Allah Taleizadeh ${ }^{1}$, Seyed Taghi Akhavan Niaki ${ }^{2, *}$ And \\ Nima AlizADEH-BASBAN ${ }^{3,4}$
}

\begin{abstract}
The high competition in today's market persuaded companies to provide some attractive options for products to obtain more market shares. Therefore, in this research, three motivating elements are devised in the framework of a two-level supply chain consisting of a manufacturer and a distributor under stochastic demand, where green production via carbon abatement, quality enhancement efforts, and returning and remanufacturing policies are used. In line with reducing carbon emission two possibilities are applied in this paper involving the green technologies investment and the trade and cap policy. As simultaneous consideration of all motivating factors raises conflicts between the members in terms of undertaking the costs, a cooperative supply chain, as well as a cost-sharing (CS) contract in non-cooperative form, is devised to alleviate the conflicts and boost the performance of the supply chain. In order to find the optimal decision variables and profits in the CS contract, two game-theoretical approaches, namely Nash and Stackelberg, are applied. The results present the sufficiently of both the cooperative chain and the CS contact. In addition, the analysis of parameters in the CS contract in Stackelberg game indicates that when the distributor undertakes a part of the costs, positive impacts on the other motivating factors are observed. However, there is no interaction among the motivating factors in the Nash game.
\end{abstract}

Mathematics Subject Classification. 90B06, 91A80, 91B70.

Received March 10, 2019. Accepted June 27, 2020.

\section{INTRODUCTION}

Offering attractive options for products has become a necessity for manufacturers in today's competitive market in order to obtain more market shares. To this aim, the companies can employ four approaches. The first approach is about attracting more customers' demands by producing sustainable products with less carbon emission $[16,34,49]$. As products with high carbon emission during their production has devastating effects on global warming and hence on human lifestyle, investment in more environmental-friendly production

Keywords. Pricing, remanufacturing, quality improvement effort, carbon emission, game theory.

1 School of Industrial Engineering, College of Engineering, University of Tehran, Tehran, Iran.

2 Department of Industrial Engineering, Sharif University of Technology, Tehran, Iran.

3 Department of Industrial Engineering, Islamic Azad University, South Tehran Branch, Tehran, Iran.

4 Department of Operations Management \& Strategy, State University of New York at Buffalo, Buffalo, NY, USA.

* Corresponding author: Niaki@Sharif.edu 
methods, equipment, and raw materials is an option to invite customers' attraction [4,52,73]. A report by H\&M, a reputable company in fashion apparel industry reveals that they have the plan to produce environmentally friendly by 2040 replacing traditional equipment and materials by green technologies, renewable energy, and materials to alleviate carbon emission [29].

The second approach to ease emission besides green equipment is to employ the trade and cap regulation; by which each company is assigned an upper limit on the amount of carbon it emits [82]. In this regulation, if a company releases more than the capacity assigned, it should pay fines for each extra amount. However, if it releases carbon less than the capacity, then the government pays incentives and it can sell the remainder to the market $[15,17]$. For instance, in China, the pollution released from the garment industry due to overconsumption of energy, textile, water during production process has become enormous. Therefore, they applied a cap and trade regulation to control emission emitted by factories [20].

The third approach involves remanufacturing of the defected products to have less undesired environmental effects as well as having economic benefits $[19,77]$. In this case, if the sold product has any defects, the customer returns it and the company pays the refund price, in order to increase the customer's tendency to purchase [42]. Afterward, the company remanufactures the returns, which in turn reduces the production costs and also decreases the carbon emission to protect the environment $[25,32]$. An epitome of the economic remanufacturing can be found in lead-acid batteries. As $70 \%$ of its make-up contains reusable energy, collecting and remanufacturing of these batteries has significant benefits [14]. More specifically, Cambridge Enterprise, as a battery recycling company, applied novel methodologies to remanufacture the lead-batteries, leading to $15 \%$ cost-saving [9].

Finally, the fourth approach is to improve the quality of the product through quality improvement efforts such as using more advanced equipment, consuming higher quality raw materials, and utilizing a better design for the production process $[24,78,80]$. For instance, based on J.D. Power's initial quality study, Ford, a reliable automobile brand, incurs costs to improve the quality of its cars, where after a long-term effort ranked in 4th top companies beating BMW, Toyota, and Hyundai [65]. It may be noted that the high implementation costs of high quality and low-carbon products besides the costs of recollecting and remanufacturing have significant effects on the price and the order quantity of the products, and consequently, on the market's share and profit of the manufacturer, the distributor, and the supply chain (SC). Therefore, employing all the above-mentioned four motivating factors in a supply chain simultaneously need handling cooperation or coordination between the members of the SC to boost the performance of the chain. To do so, the cooperative supply chains along with a cost-sharing (CS) contract are presented in this paper. The key problem of this research is on exploring how these mentioned motivating factors can enhance the supply chain's execution and to answer this question that whether or not applying the cooperative supply chain and a CS contract is sufficient. Therefore, in this research, we extend a two-stage supply chain consisting of a manufacturer and a distributor in which all the three environmental-friendly approaches including investment in low-carbon production, trade and cap, and remanufacturing of the defected products are involved. In addition, investment in the quality improvement of the products as another attractive factor is considered here. The interaction of all these factors between each other and also on the performance of the supply chain using a CS contract and cooperative form is discussed in this paper. In short, the goal of this research is to provide answers to the following questions:

- What are the optimum variables and the profits of the manufacturer, the distributor, and the supply chain?

- Can employing remanufacturing, trade and cap, investment in low-carbon as well as quality effort together increase the market's share or not?

- Are the cost-sharing contract and the cooperative supply chain effective solutions to boost the profits of the supply chain and its members?

- What are the interactions between the carbon emission rate, the price of the product, the refund price, and the quality improvement effort in a CS contract?

In the rest of the paper, the related literature review of this research is provided in Section 2, and the problem is described and formulated in Section 3. The solution methodology for a cooperative and non-cooperative supply 
chain is provided in Section 4. Computational results and related analysis to analyze the effects of the parameters and the CS contract in both the Nash and the Stackelberg games are presented in Section 5. The managerial insights of the numerical analysis are highlighted in Section 6. Finally, we conclude the paper in Section 7.

\section{LiterATURE REVIEW}

As the mainstream of this research focuses on carbon emission abatement, remanufacturing, quality of products, pricing strategy, and cooperation and coordination contracts, the following subsections are provided to discriminate between previous literature and the current work.

\subsection{Carbon emission abatement}

In regard to abating the carbon emission, two possibilities are devised by firms and government, which are respectively (a) investment in new green technologies during production and (b) employing the trade and cap regulation. The related literature of the above two possibilities is as follows.

\section{(a) Investment in low-carbon technologies}

Due to the detrimental impacts of the carbon emission on human lifestyle, a number of companies applied new green technologies and equipment in their production flow to not only reduce the amount of emission but also motivate the consumers to purchase the products more. There are some researchers such as Hong and Guo [30], Ma et al. [47], Song and Gao, [60], Albuquerque et al. [1], Rodríguez et al. [55], Benjaafar et al. [3], Swami and Shah [59], Jin et al. [37], Luo et al. [46], Zhang and Xu [82], Böttcher and Müller [6], Bi et al. [5], Fernando et al. [18] and Wang et al. [75] in the literature who focused on low-carbon technologies to abate emission. For instance, Benjaafar et al. [3] examined the effects of investment in new technologies to produce products with a lower amount of carbon emissions for the first time. They analyzed how producing green products can stimulate the market share and increase the supply chain's profit. Ma et al. [47] considered a supply chain consisting of two manufacturers and one retailer in which one of the manufacturers invests in new technologies to produce green products. While the demand for the market was assumed certain in their research, they employed sixgame models to find optimal decision variables and the supply chain's profit. Swami and Shah [59] analyzed the effects of low-carbon production on the profit of a two-stage supply chain with a retailer and a manufacturer in which demand of the market was deterministic. They used a Stackelberg game to attain the optimum values of the decision variables. Hong and Guo [30] employed new technologies to produce low-carbon production in a two-stage supply chain including one distributor and one manufacturer. They used the Stackelberg game to obtain closed-form equations to determine the optimum values of the decision variables in which demand of the market was uncertain.

The aforementioned works just focused on switching facilities and materials in the production process into environment-friendly ones to abate the amount of carbon emission. However, they did not consider the role of government in the production process. In the following subsection, some works conducted on the use of a governmental policy to ease carbon emission are reviewed.

\section{(b) Trade and cap regulation}

Various policies such as governmental tariffs [27,28,35,48,58], carbon tax [16,85], and trade and cap regulation $[11,15,43,66,72,74,76]$ have been used by governments to intervene in the production process of the firms in order to control the amount of carbon emission. This research focuses on trade and cap policy to reduce carbon emission. Wang et al. [74] used the trade and cap regulation alongside investment in green products to reduce the carbon emission during production in a two-stage supply chain. They used Nash and Stackelberg games model to find optimal solutions in which demand of the market was stochastic. Wang et al. [76] employed the cap and trade policy in the fresh food industry to analyze the impacts of this governmental regulation on the profit of the supply chain. They assumed a deterministic demand and used a Stackelberg game to obtain the optimal solutions. Li et al. [43] analyzed the impacts of the cap and trade regulation on the amount of carbon emitted 
in a two-stage supply chain in which demand of the market was uncertain. They utilized the Stackelberg game to obtain the optimal values of the decision variables. Cheng and Zhang [11] considered a two-level supply chain consisting of one manufacturer and one retailer in which retailer sells the products either traditional or online. They applied the cap and trade regulation to ease the carbon amount. Moreover, they used the Stackelberg game to find the optimal profits of the supply chain and its members in which demand of the market was deterministic. Tong et al. [72] considered the Chinese appliance industry in which trade policy besides green investment in production process was devised to eliminate carbon footprint. They used an evolutionary game in the retailer dominant case and obtained the optimum amount of carbon and also the optimal price of the product to maximize SC profit and preserve the environmental issues.

Although the previous works applied various governmental policies as well as green technological investments to ease carbon emission and attract the market share, they did not consider the performance of the green factor besides other motivational elements in the competitive market simultaneously. Here, other marketing variables including the suitable pricing strategy, quality improvement effort, as well as collecting and remanufacturing of defects are involved to enhance the market share in presence of various game-theoretical approaches, which have not been investigated in previous research works.

\subsection{Returning and remanufacturing the defected products}

As a motivation factor to raise market share, companies paying the compensated price to customers to collect and remanufacture the defected products. There are various methodologies to collect the returns, including via either the upstream or downstream members of the SC $[32,42,53,57,61,64,81]$ and dual recycling via both downstream and upstream members simultaneously $[33,35,66,69]$. In this paper, a single-collecting approach is focused to collect and remanufacture products, not dual recycling. Huang and Wang [32] defined three different single-collecting approaches to remanufacture the deteriorated products, including via manufacturer only, via distributor only, and via a third party. They used the Stackelberg game under deterministic demand to comprise between recollecting approaches. Their results showed that using dual-remanufacturing is more beneficial rather than single one. Örsdemir et al. [53] considered a two-level supply chain in which a manufacturer produces new products and a retailer remanufactures the returned products. The quality of new products and remanufactured ones were not the same and there was a competition between the members to sell their products. They used the Nash game to find the optimal order quantity of the new and the remanufactured products. Zerang et al. [81] examined a three-level supply chain including a third party, a manufacturer and a retailer to remanufacture the returns. The third-party was responsible to collect the defected products and to send them back to the manufacturer. Meanwhile, the manufacturer was responsible to remanufacture the returns with the quality as the new one. They employed the Stackelberg game to find solutions under a deterministic demand. Other related research in this area can be found in Taleizadeh and Noori-daryan [63], Taleizadeh et al. [67], Taleizadeh et al. [71], and Moshtagh and Taleizadeh [50].

Although previous literature focused on employing various reverse channels, either single or dual, to remanufacture the products, they did not consider remanufacturing impacts on the performance of the SC in presence of other motivation factors including carbon abatement possibilities, quality enhancement effort, suitable pricing strategy. In addition, implementation of such a complex SC needs to incur considerable costs, persuading manufacturer to make coordination among the downstream members to alleviate costs. Therefore, a CS contract is employed in this paper to increase the implementation possibility of this SC, which has not been discussed in the literature previously.

\subsection{Quality improvement effort}

In addition to carbon abatement alongside returning and remanufacturing defective products, quality of the products is another important motivating factor discussed in the literature to attract market share $[38,39,44$, $56,79,80]$. For instance, Xie et al. [79] studied the effect of quality improvement efforts in an SC involving two retailers and two manufacturers. They assumed certain demand and applied the Nash game and cooperation 
among members vertically and horizontally. They showed that using vertical cooperation has better performance rather than other games. Xiong [80] analyzed the role of quality in a two-echelon supply chain consisting of a manufacturer and a retailer. They employed the Stackelberg game under deterministic demand to find out the interaction between the quality and the price. Their results showed that the quality improvement effort can increase customer demand and consequently can raise the profit of the SC. As improving the quality leads to a higher product price, a revenue-cost sharing contract was used in this research, making coordination between the members. Li et al. [44] analyzed the impacts of quality on the profit of a two-stage supply chain involving a supplier and a retailer. They considered both centralized and decentralized structures, where Nash bargaining was used in the decentralized form. They showed that the profit of SC drastically depends on the sensitivity of the customers to price and the quality of the products.

Besides the quality improvement efforts, there are also scant works in the literature that considered other motivating factors such as the return policy, the coordination contracts, and various selling channels to attract market share and to study the interaction between the factors. Li et al. [42] examined the relationship between the return policy and the quality level as two motivating factors. They obtained optimal refund price and quality under certain demand, where high quality of products diminished the quantity of defected products, and consequently, enhanced the profit of manufacturer. Jingxian et al. [38] investigated the impacts of the quality and price on profit of a two-level supply chain, where the product delivered to customers via the manufacturer directly, via the traditional retail channel, and via a dual channel. They obtained the optimal values of the decision variables in both centralized and decentralized forms, in which adding new selling channel enhanced the profit of the SC. Zhang and Hong [83] studied the interaction between the quality improvement effort and the carbon emission abatement cost in a two-stage supply chain consisting of a supplier and a manufacturer under certain demand. Regarding the high costs of quality enhancement, they employed three coordination contracts comprised of revenue sharing, cost-sharing, and wholesale price. They obtained the optimum price and the quality level in each contract using the Stackelberg game. Furthermore, they illustrated that all coordination contracts are sufficient to boost the profit of SC.

Although previous literature studied the effects of quality improvement efforts on the price and the profit of supply chains in the framework of game-theoretical approaches, they did not analyze efficiently the impact of quality improvement effort in the presence of other motivating factors including carbon reduction (green technology investment, trade and cap policy), returning and remanufacturing of defects. In addition, applying all aforementioned elements to attract market share impose costs. Therefore, in order to alleviate the conflicts between the members of such complex SC, cost-sharing contracts under various game structures (Nash and Stackelberg) are devised in this paper, which has not been applied previously. Recently, Taleizadeh et al. [64] considered interactions between the quality improvement efforts, the carbon footprint reduction and return policy in a two-level supply chain under stochastic demand, where the price of products was assumed fixed. They did not consider the impacts of high implementation costs of motivating factors on the price and the demand. However, in the current research, the product price is assumed a decision variable and in order to find the optimal price, a CS contract in both Nash and Stackelberg games is applied.

\subsection{Cooperation and coordination contracts}

As implementing motivating factors to boost market demand imposes a significant cost, it is a point of argument between the members. Therefore, applying cooperation and coordination contracts are effective solutions to boost the performance of the supply chain. These contracts were considered in previous literature such as Giri et al. [23], Song and Gao [60], Taleizadeh et al. [66], Wang et al. [74], Cao and Yu [8]. For instance, Taleizadeh et al. [66] expanded a two-stage supply chain in which the cap and trade regulation and low-carbon investment were employed to reduce carbon emission. In their work, the demand for the market was stochastic and depended on the price and the carbon emission rate. They used Nash and Stackelberg games to attain optimum solutions. Besides, in order to make coordination between the members, a cost-sharing, a buyback and a wholesale price contract along with a cooperative supply chain was used. They highlighted that using the wholesale contract in both Nash and Stackelberg games was more fruitful rather than the others. Giri et al. [23] 
analyzed a two-stage supply chain involving a retailer and two manufacturers whose products had different qualities and return policy was employed to attract the market share. In order to alleviate conflicts between the manufacturers, they assumed a revenue-sharing contract under the Nash game. They showed that the contract was an effective approach. Cao and $\mathrm{Yu}[8]$ assumed a two-stage supply chain in both cooperative and noncooperative forms. They used the cap and trade regulation to reduce the carbon emission in which demand of the market was uncertain and depended on the carbon emission rate and the price of the product. They employed revenue sharing, quantity discounts, and buyback contracts to make coordination between the members using the Stackelberg game. They showed that using these contracts can coordinate SC under some restrictions. Song and Gao [60] used the revenue sharing contract to boost the performance of a two-stage supply chain. The demand of the market was certain and depended on the carbon emission rate and the price of the products. They used a Stackelberg game to find closed-form equations to obtain the variables and profits. The results illustrated that using the revenue sharing contract was fruitful. Some other related research can be found in Taleizadeh [68], Taleizadeh et al. [68,70,71], Lashgari et al. [40,41], and Pourmohammad Zia and Taleizadeh [54].

Although previous researchers employed various cooperation and coordination contracts to enhance the profitability of an SC in presence of motivating factors, there is no paper in the literature that uses coordination contracts in the presence of various motivating elements simultaneously. In this research, however, in line with increasing the market demand, carbon abatement possibilities (the green technologies investment and trade and cap regulation), collecting defective products by paying the refund price, remanufacturing, and quality enhancement effort are applied simultaneously under stochastic demand. Moreover, high costs of implementation persuade the SC to use coordination contract (cost-sharing contract) to ease conflicts between the members and to boost the performance of the chain.

\subsection{Research gap}

In spite of highlighting the difference between previous papers and what this research is aiming in each of the above subsection of the literature review, a comparative assessment is provided in Table 1 to clarify the contribution of this paper. This research is an extension of the work by Taleizadeh et al. [64] and Wang et al. [74], in which three motivating elements in a two-stage supply chain under the stochastic demand are defined. The work comprises the green technologies investment, trade and cap regulation, quality enhancement effort, refund policy, and remanufacturing. Nash and Stackelberg games are used to obtain the closed-form of optimal decision variables in both centralized and decentralized structures. Moreover, due to high costs of this complex SC, a cost-sharing contract in both Nash and Stackelberg game is employed to make coordination between the members and to boost the profit of the SC. Although Wang et al. [74] considered cap and trade besides green investment to reduce carbon in a supply chain under the stochastic demand and used wholesale price and cost-sharing contracts to make coordination, they did not add other motivating factors including quality, return policy, and remanufacturing simultaneously. This would increase the complexity of finding the optimum values of the decision variables. In addition, they assumed fixed price to alleviate high costs of the carbon abatement implementation, while in our research the price is determined through a decision variable. Recently, Taleizadeh et al. [64] studied the interactions of the quality improvement effort, remanufacturing, and carbon abatement in the framework of a decentralized SC under stochastic demand. However, they employed a different collecting policy. They assumed that the downstream member was responsible to pay the refund price and to collect the defected products and send them back to the manufacturer for recycling. Nonetheless, in the current paper the manufacturer is held responsible for collecting and remanufacturing all by himself. In addition, they did not provide any solution for high costs of implementation of such a complex SC. Imposing costs significantly affects the price of the product, which may be in contrast to the customers' desire. Therefore, a CS contract is employed in the current work in both Nash and Stackelberg games as well as considering a centralized structure to obtain the optimal values of the decision variables to maximize the profit of the SC. Taleizadeh et al. [64] just used the Stackelberg game in a decentralized case to achieve the optimum profit. In addition, they considered the price of the product as a fixed parameter to reduce the solution complexity, whilst we assume the price 


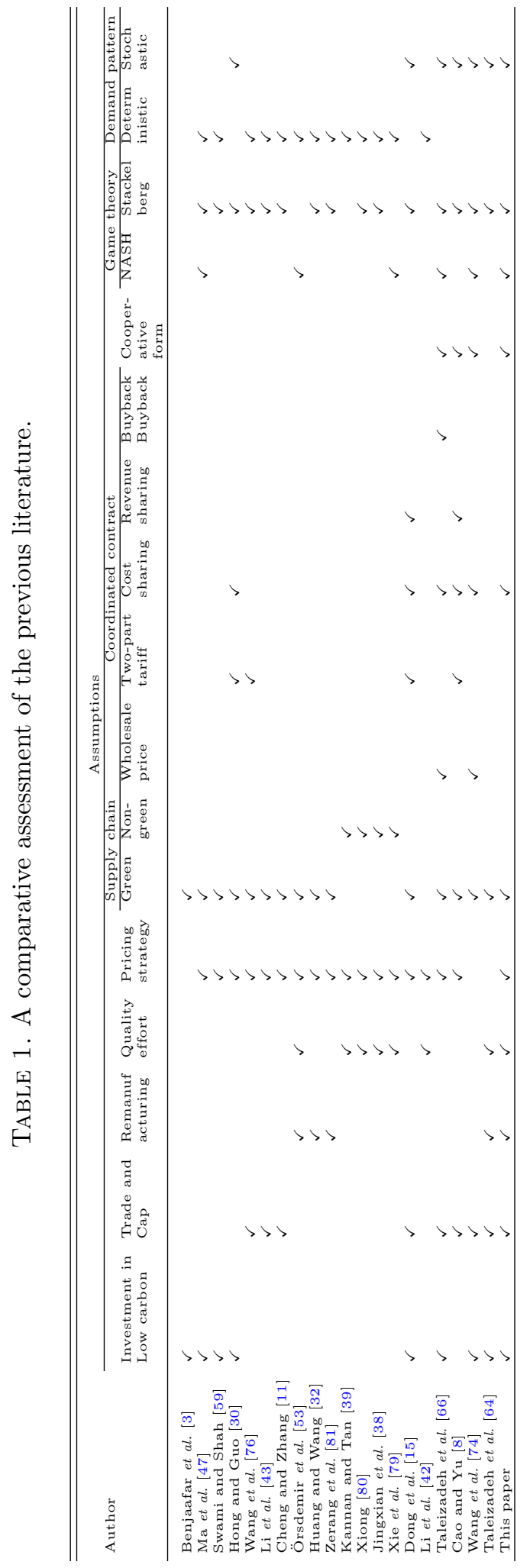


a decision variable. Moreover, the interaction between the price and the carbon emission and the refund price are analyzed in this paper, which has not been addressed in the literature.

\section{Problem Description}

In this paper, a two-stage supply chain consisting of a distributor (R) and a manufacturer (M) is assumed in which the motivating factors including low-carbon production, quality improvement, and setting the suitable price are involved to increase the market share of the supply chain. In order to reduce the carbon emission, two possibilities including the trade and cap policy and the investment in new production technologies are considered. In the trade and cap regulation, the government imposes a restriction of emitting carbon less than an assigned capacity $\left(L_{s}\right)$ on all the manufacturers. In case the carbon emission level of the manufacturer is less than $L_{s}$, the residual capacity can be sold to other manufacturers at the price $p_{s}$ per each emitted carbon unit. However, when the manufacturer releases carbon above the limit $L_{s}$, he has to pay a penalty $p_{s}$ for each excess carbon unit. Therefore, the effects of all two reduction possibilities are considered in the carbon emission reduction rate $(s)$ in the interval $0 \leq s<1$, because it is assumed that the manufacturer cannot reduce all amounts of the emission and that if the manufacturer does not reduce the carbon emission at all, the reduction rate would be zero. On the other hand, collecting and remanufacturing defective products is another motivating factor to attract market share and to save costs. In the remanufacturing's flow, the manufacturer pays a refund price $(r)$ to the customers for each unit of defective products. All the returned products are assumed to be remanufactured successfully and that there is no difference between the new and the remanufactured products $[12,21,22,31,64]$. In this paper, the manufacturer is responsible for investment in the low-carbon production, the quality improvement and recollecting of the defective products. Meanwhile, the distributor is responsible to assign the optimal final price and the optimal order quantity of the products. In addition, the implementation of a supply chain considering all mentioned factors needs cooperation and coordination between the members to boost the profit of the chain, the distributor and the manufacturer. Therefore, a cooperative supply chain using a CS contract is applied in a non-cooperative form.

The schematic diagram of the material's flow in this research is drawn in Figure 1.

\subsection{Notation}

The following notations are used to express the mathematical model of the problem under investigation.

\section{(a) Parameters}

$C_{m} \quad$ The unit production cost of a new product.

$C_{r} \quad$ The unit remanufacturing cost of a returned product $\left(C_{r}<C_{m}\right)$.

$T \quad$ Savings per unit of the sold remanufactured returned product.

$\alpha \quad$ Fix quantity of the returned products.

$\delta \quad$ The positive impact coefficient of the refund price on the quantity of the returned products.

$\gamma \quad$ The negative impact coefficient of the quality on the quantity of returned products.

$\lambda \quad$ Carbon releasing rate per unit of the product.

$L_{s} \quad$ The governmentally-assigned upper limit of carbon emission per manufacturer.

$p_{s} \quad$ The unit price of carbon emission to sell or pay in governmental regulation.

$\theta \quad$ The investment coefficient to improve the quality level.

$\phi \quad$ The cost coefficient to reduce the carbon emission. 


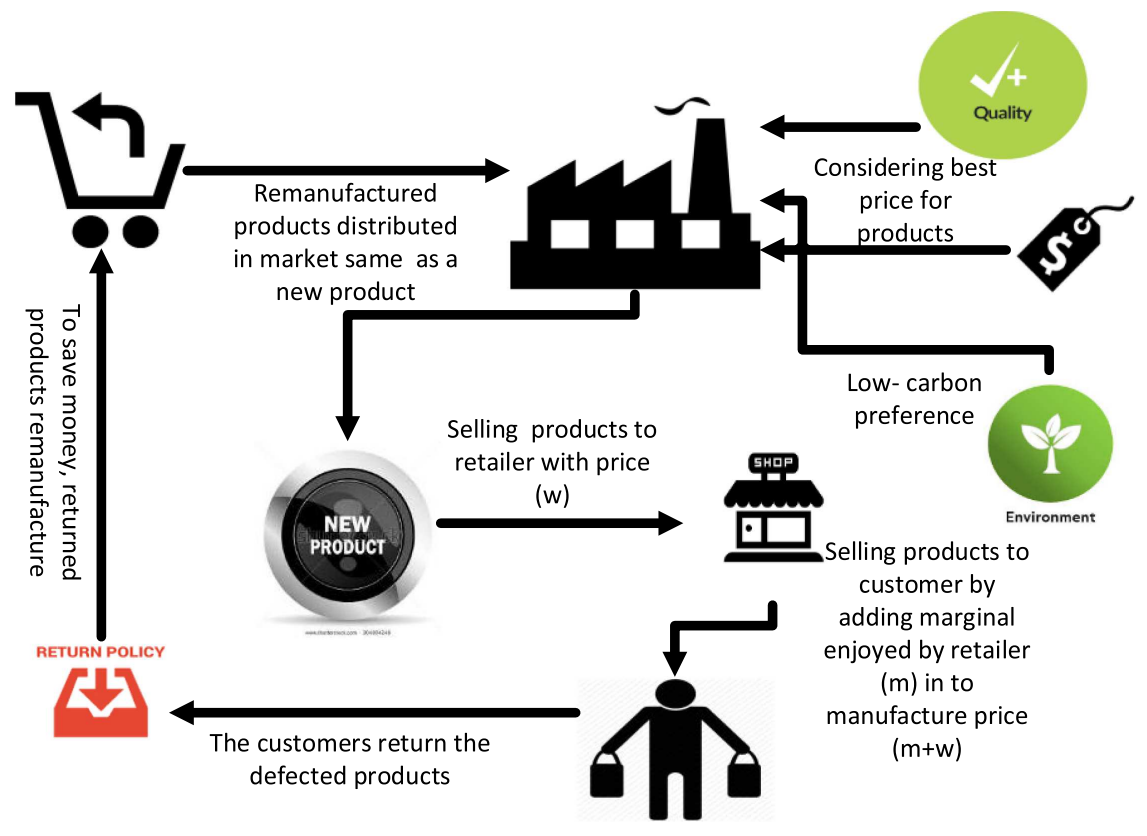

FIGURE 1. The supply chain system with remanufacturing, pricing, quality effort, and carbon emission.

$\mu \quad$ The mean of the uniform distribution function of the error term.

$\Gamma \quad$ The rate of cost-sharing for quality improvement undertaken by the distributor.

$\Delta \quad$ The rate of cost-sharing for low-carbon production undertaken by the distributor.

$f($.$) \quad The probability density function.$

$F($.$) \quad The cumulative probability density function.$

$O \quad$ The order or the production quantity of the manufacturer.

SR, N, C Denote respectively, Stackelberg in retailer's dominant case, Nash game, and the cooperative supply chain.

$\mathrm{SC}, \mathrm{M}, \mathrm{R}$ Show the supply chain, the manufacturer, and the distributor, respectively.

\section{(b) Intermediate variables}

$L(O) \quad$ The expected sale quantity of the distributor.

$Z(O)$ The expected leftover inventory.

$S(O) \quad$ The distributor lost-sales.

\section{(c) Decision variables}

$w$ The unit wholesale price of the product proposed by the manufacturer $\left(w \geq C_{m}\right)$.

$p \quad$ Unit final price of the product proposed by the distributor $(p \geq w)$.

$s \quad$ The rate of carbon reduction as an environmentally sustainable factor $(0 \leq s<1)$.

$r$ Unit refund price of returned products paid by the manufacturer to the customer $(r \leq w \leq p)$.

$q \quad$ The quality level of the product.

$m$ Unit marginal profit of the product enjoyed by the distributor $(m>0)$.

I Anticipated inventory of the distributor. 


\section{(d) Performance measures}

$\Pi_{\mathrm{C}} \quad$ The cooperative supply chain profit.

$\Pi_{\mathrm{M}} \quad$ The manufacturer profit.

$\Pi_{\mathrm{R}} \quad$ The distributor profit.

\subsection{Problem formulation}

The market demand of the supply chain is assumed stochastic and is determined using equation (3.1) (similar to $[15,59,66,74])$ :

$$
D(s, p, r, q)=\beta_{0}-\beta_{1} p+\beta_{2} s+\beta_{3} r+\beta_{4} q+\varepsilon,
$$

where $\beta_{0}$ is the base demand that is independent of the final price, carbon emission, refund price, and the quality effort of the product, $\beta_{1}$ is the impact coefficient of the final price on the market demand, $\beta_{2}$ is the impact coefficient of the carbon reduction rate on the market demand, $\beta_{3}$ is the impact coefficient of the refund price, and $\beta_{4}$ is impact coefficient of the quality effort of the market demand. In addition, $\varepsilon$ is the error term, which follows a uniform distribution function in the interval $[0, E]$ with a mean $(\mu)$ with the cumulative probability density function denoted by $F(\varepsilon)$. Note that the error term is added to guarantee that the demand of the market is uncertain.

Moreover, the cost function of investment in reduction of carbon emission during production (similar to $[36,45]), C(s)$, and the cost function of the quality effort (similar to $[10,42]), C(q)$, are assumed in the following forms, respectively.

$$
\begin{aligned}
& C(s)=\phi\left(s^{2} / 2\right) \\
& C(q)=\theta q^{2},
\end{aligned}
$$

where $\theta$ is the investment coefficient to improve the quality level and $\phi$ is the cost coefficient to reduce the carbon emission rate.

Similar to Atasu et al. [2] and Huang and Wang [32], the saving obtained by selling a unit of remanufactured product, $T$, is obtained by:

$$
T=C_{m}-C_{r},
$$

where $C_{m}$ and $C_{r}$ are the unit production costs of a new and a remanufactured product, respectively. Furthermore, the quantity of the returned product (similar to [42]) is assumed as a linear function of the refund price $(r)$ and the quality improvement effort of the product $(q)$ defined in equation (3.5).

$$
Q(r, q)=\alpha+\delta r-\gamma q,
$$

where $\alpha$ is a fixed number of the returned products being independent of the refund price and the quality effort of the product, $\delta$ is the refund price coefficient, and $\gamma$ is the coefficient of the quality improvement effort of the returned products.

According to what described so far, the profit function of the manufacturer is formulated as:

$$
\Pi_{\mathrm{M}}=\left(w-c_{m}\right)(O-Q(r, q))+\left(w-c_{m}+T-r\right) Q(r, q)-p_{s}\left[\lambda(1-s) O-L_{s}\right]-\phi \frac{s^{2}}{2}-\theta q^{2} .
$$

Note in equation (3.6) that no shortage cost, salvage value, and holding cost is assumed for the manufacturer's profit function. In addition, the expected distributor's profit function is

$$
E\left(\prod_{\mathrm{R}}\right)=p L(O)-w O-h^{+} Z(O)-h^{-} S(O),
$$


where $h^{+}$is the unit holding cost of unsold products and $h^{-}$denotes the unit shortage cost. In addition, the expected sale quantity $L(O)$, the expected leftover inventory $Z(O)$, and lost sales of the retailer $S(O)$ are derived using equations (3.8)-(3.10), respectively.

$$
\begin{aligned}
L(O) & =O[1-F(O)]+\int_{0}^{O} f(D) D \mathrm{~d} D=O-\int_{0}^{O} F(D) \mathrm{d} D \\
Z(O) & =(O-D)^{+}=O-L(O) \\
S(O) & =(D-O)^{+}=E(D)-L(O) .
\end{aligned}
$$

The market demand has two parts: (1) $\beta_{0}-\beta_{1} p+\beta_{2} s+\beta_{3} r+\beta_{4} q$ as a certain term and (2) $\varepsilon$ as the stochastic term. It is inevitable that the customers will order at least as much as a certain term, but in order to address the error term, an auxiliary variable, $I$, is added to the market demand as the anticipated inventory level of the distributor. As such, we have:

$$
O=I+\beta_{0}-\beta_{1} p+\beta_{2} s+\beta_{3} r+\beta_{4} q .
$$

Note in equation (3.11) that if $I>\varepsilon$, then the residual inventory will remain and if $I<\varepsilon$ the shortage will occur. Therefore, the substitution of equation (3.11) instead of $O$ in the distributor's profit function (3.7) leads to:

$$
\begin{aligned}
E\left(\prod_{\mathrm{R}}(p, I)\right)= & {\left[(p-w)\left(I+\beta_{0}-\beta_{1} p+\beta_{2} s+\beta_{3} r+\beta_{4} q\right)\right]+h^{-}(I-\mu) } \\
& -\left(p+h^{+}+h^{-}\right) \int_{0}^{I} F(\varepsilon) \mathrm{d} \varepsilon .
\end{aligned}
$$

In what follows in the next sections the manufacturer's and the distributor's profits are formulated in different cases, where the solution methodologies are provided.

\section{Solution methodology}

The cooperative and non-cooperative forms of the supply chain are modeled and solved in this section to find the optimal values of the decision variables of the manufacturer (the wholesale price, the quality effort, the carbon reduction rate, and the refund price) and the retailer (the final price and the anticipated inventory level). In non-cooperative form, the game-theoretic approaches including Nash and Stackelberg games in distributor's dominant case are used to obtain the closed-form equations to determine the decision variables. In the latter case, a CS contract in both games is used to coordinate the non-cooperative supply chain, based on which the profits of the chain members and the closed-form equations of the decision variables are derived.

\subsection{Cooperative supply chain}

In the cooperative form, the manufacturer integrates with the distributor to be a united part to improve the supply chain's performance. In this form, both the manufacturer and the distributor have the same goal to maximize the profit function of the supply chain and find the optimum values of the final price $(p)$, the carbon emission reduction rate $(s)$, the quality effort $(q)$, the refund price $(r)$, and the distributor's inventory level $(I)$. The supply chain's profit, in this case, is formulated as:

$$
\begin{aligned}
\operatorname{Max} E\left(\prod_{\text {SC }}(s, r, q, p, I)\right)= & {\left[\left(p-c_{m}\right)\left(I+\beta_{0}-\beta_{1} p+\beta_{2} s+\beta_{3} r+\beta_{4} q\right)\right]+h^{-}(I-\mu) } \\
& +(T-r)(\alpha+\delta r-\gamma q)-\phi \frac{s^{2}}{2}-\theta q^{2}-\left(p+h^{+}+h^{-}\right) \int_{0}^{I} F(\varepsilon) \mathrm{d} \varepsilon \\
& -p_{s}\left[\lambda(1-s)\left(I+\beta_{0}-\beta_{1} p+\beta_{2} s+\beta_{3} r+\beta_{4} q\right)-L_{s}\right] .
\end{aligned}
$$


Theorem 4.1. The optimal values of the decision variables in the cooperative supply chain are obtained using the following equations:

$$
\begin{aligned}
q^{\mathrm{C}} & =\frac{-A_{11} p^{\mathrm{C}}-A_{12} s^{\mathrm{C}}-A_{14}}{A_{13}} \\
r^{\mathrm{C}} & =\frac{-A_{15} p^{\mathrm{C}}-A_{16} s^{\mathrm{C}}-A_{17}}{\gamma} \\
s^{\mathrm{C}} & =\frac{-A_{18} p^{\mathrm{C}}-A_{5} I^{\mathrm{C}}-A_{20}}{A_{19}} \\
I^{\mathrm{C}} & =\frac{2 A_{22} E}{3}+\frac{A_{24} E}{3}-\frac{H_{1}}{\left(H_{2}-H_{3}+H_{5}\right)^{1 / 3}}+\left(H_{2}-H_{3}+H_{5}\right)^{1 / 3} \\
p^{\mathrm{C}} & =\frac{-2 A_{23} E-2 A_{22} E I^{\mathrm{C}}+\left(I^{\mathrm{C}}\right)^{2}}{2 A_{21} E} .
\end{aligned}
$$

Proof. See Appendix A.

\subsection{Non-cooperative supply chain}

In the non-cooperative supply chain, the manufacturer and the distributor attempt to maximize their own profits separately. In other words, the manufacturer determines the optimal values of the carbon emission reduction rate, the quality effort, the unit wholesale price, and the unit refund price of the product in order to maximize his own profit. Meanwhile, the distributor assigns the optimal values of the final price and the inventory level using both the Nash and the Stackelberg games. In what follows, closed-form equations are obtained for the optimal values of the decision variables in Nash and Stackelberg games.

\section{(a) Nash game analysis}

In the Nash game, the choice powers of the manufacturer and the distributor are the same and each member tries to maximize his/her own profit function by finding the optimal values of decision variables. The members find their optimum variables simultaneously. In the Nash game, the optimal values of the manufacturer's decision variables are obtained maximizing equation (3.6) with respect to the decision variables. Note in this equation that the profit function of the manufacture is linear in terms of $w$. Therefore, similar to Zhao et al. [84] and Taleizadeh et al. [66] without loss of generality, the unit final price of the product is substituted with

$$
p=m+w,
$$

where $m>0$ is the unit marginal profit received by the distributor for each sold product. Equation (4.7) enables one to first obtain a closed-form equation for the unit wholesale price and then for the unit final price of the product.

Theorem 4.2. The optimal values of the manufacturer's decision variables in the Nash game are:

$$
\begin{aligned}
r^{\mathrm{N}} & =\frac{-B_{11} w^{\mathrm{N}}-B_{12} s^{\mathrm{N}}+B_{14}}{B_{13}} \\
q^{\mathrm{N}} & =\frac{B_{15} w^{\mathrm{N}}+B_{16} s^{\mathrm{N}}+B_{17}}{2 \theta} \\
s^{\mathrm{N}} & =\frac{B_{25} m^{\mathrm{N}}-B_{26} I^{\mathrm{N}}-B_{27}}{B_{24}} \\
w^{\mathrm{N}} & =\frac{B_{28} m^{\mathrm{N}}-B_{29} I^{\mathrm{N}}-B_{30}}{B_{21}} .
\end{aligned}
$$


Proof. See Appendix B.

Meanwhile, the optimal values of the decision variables of the distributor are presented in the following theorem.

Theorem 4.3. The closed-form equations for the optimal anticipated inventory level and the optimal unit marginal profit of the distributor in Nash game are:

$$
\begin{gathered}
I^{\mathrm{N}}=\frac{1+2 E B_{32} B_{34}+2 E B_{31} B_{35}}{3 B_{34}}+\frac{F_{1}-F_{2}}{F_{7}}+F_{7} \\
m^{\mathrm{N}}=\frac{-2 E B_{33}-2 E B_{32} I^{\mathrm{N}}+\left(I^{\mathrm{N}}\right)^{2}}{2 E B_{31}} .
\end{gathered}
$$

Proof. See Appendix C.

\section{(b) Stackelberg game in distributor's dominant case}

According to the backward solution in the Stackelberg game in which the distributor plays the dominant role, the manufacturer first obtains the optimal values of his decision variables in order to maximize his profit. Then, the optimal values of the manufacturer's decision variables are substituted into the distributor's profit function (3.12) to find the optimal setting of the retailer's decision variables.

Theorem 4.4. The closed-form equations for the optimal values of the manufacturer's decision variables in the Stackelberg game are:

$$
\begin{aligned}
s^{\mathrm{SR}} & =\frac{B_{25} m^{\mathrm{SR}}-B_{26} I^{\mathrm{SR}}-B_{27}}{B_{24}} \\
w^{\mathrm{SR}} & =\frac{B_{28} m^{\mathrm{SR}}-B_{29} I^{\mathrm{SR}}-B_{30}}{B_{21}} \\
r^{\mathrm{SR}} & =\frac{-B_{11} w^{\mathrm{SR}}-B_{12} s^{\mathrm{SR}}+B_{14}}{B_{13}} \\
q^{\mathrm{SR}} & =\frac{B_{15} w^{\mathrm{SR}}+B_{16} s^{\mathrm{SR}}+B_{17}}{2 \theta} .
\end{aligned}
$$

The proof of Theorem 4.4 is as similar to what is presented in proof of Theorem 4.2 in Appendix B.

Afterward, the optimal decision variables of the manufacturers shown in equations (4.14)-(4.17) are replaced into the distributor's profit function (3.12) to find the optimum variables of distributor under the Stackelberg game in distributor's dominant case. Hence, the profit function of the distributor is rearranged as:

$$
\begin{aligned}
\prod_{\mathrm{R}}^{\mathrm{SR}}=m\left[\begin{array}{l}
\left(-\beta_{1}-\frac{\beta_{1} B_{28}}{B_{21}}-\frac{\beta_{2} B_{25}}{B_{24}}-\frac{\beta_{3} B_{11} B_{28}}{B_{13} B_{21}}+\frac{\beta_{3} B_{12} B_{25}}{B_{13} B_{24}}+\frac{\beta_{4} B_{15} B_{28}}{2 \theta B_{21}}-\frac{\beta_{4} B_{16} B_{25}}{2 \theta B_{24}}\right) m \\
+\left(\begin{array}{l}
\beta_{1} B_{29} \\
B_{21}
\end{array}-\frac{\beta_{2} B_{26}}{B_{24}}+\frac{\beta_{3} B_{11} B_{29}}{B_{13} B_{21}}+\frac{\beta_{3} B_{12} B_{26}}{B_{13} B_{24}}-\frac{\beta_{4} B_{15} B_{29}}{2 \theta B_{21}}-\frac{\beta_{4} B_{16} B_{26}}{2 \theta B_{24}}+1\right) I \\
+\left(\beta_{0}+\frac{\beta_{1} B_{30}}{B_{21}}-\frac{\beta_{2} B_{27}}{B_{24}}+\frac{\beta_{3} B_{11} B_{30}}{B_{13} B_{21}}+\frac{\beta_{3} B_{12} B_{27}}{B_{13} B_{24}}+\frac{\beta_{3} B_{14}}{B_{13}}-\frac{\beta_{4} B_{15} B_{30}}{2 \theta B_{21}}-\frac{\beta_{4} B_{16} B_{27}}{2 \theta B_{24}}+\frac{\beta_{4} B_{17}}{2 \theta}\right)
\end{array}\right] \\
+
\end{aligned}
$$

Theorem 4.5. The optimal values of the distributor's decision variables in the Stackelberg game are given in equations (4.19) and (4.20).

$$
\begin{aligned}
I^{\mathrm{SR}} & =S_{16}+\frac{2 S_{1} S_{10}+S_{2} S_{7}+S_{2} S_{8}}{3 S_{7} S_{8}}+\frac{S_{11}}{S_{16}} \\
m^{\mathrm{SR}} & =\frac{-S_{3}-S_{2} I^{\mathrm{SR}}+S_{7}\left(I^{\mathrm{SR}}\right)^{2}}{2 S_{1}} .
\end{aligned}
$$


TABLE 2. Decision variables in the cooperative and non-cooperative supply chains.

\begin{tabular}{llll}
\hline \hline $\begin{array}{l}\text { Decision } \\
\text { vari- } \\
\text { ables }\end{array}$ & Centralized & Nash & Stackelberg \\
\hline$(s)$ & $\frac{-A_{18} p^{\mathrm{C}}-A_{5} I^{\mathrm{C}}-A_{20}}{A_{19}}$ & $\frac{B_{25} m^{\mathrm{N}}-B_{26} I^{\mathrm{N}}-B_{27}}{B_{24}}$ & $\frac{B_{25} m^{\mathrm{SR}}-B_{26} I^{\mathrm{SR}}-B_{27}}{B_{24}}$ \\
$(r)$ & $\frac{-A_{15} p^{\mathrm{C}}-A_{16} s^{\mathrm{C}}-A_{17}}{\gamma}$ & $\frac{-B_{11} w^{\mathrm{N}}-B_{12} s^{\mathrm{N}}+B_{14}}{B_{13}}$ & $\frac{-B_{11} w^{\mathrm{SR}}-B_{12} s^{\mathrm{SR}}+B_{14}}{B_{13}}$ \\
$(q)$ & $\frac{-A_{11} p^{\mathrm{C}}-A_{12} s^{\mathrm{C}}-A_{14}}{A_{13}}$ & $\frac{B_{15} w^{\mathrm{N}}+B_{16} s^{\mathrm{N}}+B_{17}}{2 \theta}$ & $\frac{B_{15} w^{\mathrm{SR}}+B_{16} s^{\mathrm{SR}}+B_{17}}{2 \theta}$ \\
$(w)$ & $-\quad \frac{B_{28} m^{\mathrm{N}}-B_{29} I^{\mathrm{N}}-B_{30}}{B_{21}}$ & $\frac{B_{28} m^{\mathrm{SR}}-B_{29} I^{\mathrm{SR}}-B_{30}}{B_{21}}$ \\
$(m)$ & $-\quad$ & $\frac{-2 E B_{33}-2 E B_{32} I^{\mathrm{N}}+\left(I^{\mathrm{N}}\right)^{2}}{2 E B_{31}}$ & $\frac{-S_{3}-S_{2} I^{\mathrm{SR}}+S_{7}\left(I^{\mathrm{SR}}\right)^{2}}{2 S_{1}}$ \\
$(p)$ & $\frac{-2 A_{23} E-2 A_{22} E I^{\mathrm{C}}+\left(I^{\mathrm{C}}\right)^{2}}{2 A_{21} E}$ & $\frac{B_{28} m^{\mathrm{N}}-B_{29} I^{\mathrm{N}}-B_{30}}{B_{21}}$ & $\frac{B_{28} m^{\mathrm{SR}}-B_{29} I^{\mathrm{SR}}-B_{30}}{B_{21}}$ \\
& $\frac{2 A_{22} E}{3}+\frac{A_{24} E}{3}-\frac{-2 E B_{33}-2 E B_{32} I^{\mathrm{N}}+\left(I^{\mathrm{N}}\right)^{2}}{2 E B_{31}}$ & $+\frac{-S_{3}-S_{2} I^{\mathrm{SR}}+S_{7}\left(I^{\mathrm{SR}}\right)^{2}}{2 S_{1}}$ \\
$(I)$ & $+\left(H_{2}-H_{3}+H_{5}\right)^{1 / 3}$ & $\frac{1+2 E B_{32} B_{34}+2 E B_{31} B_{35}}{3 B_{34}}$ & \\
\hline
\end{tabular}

Proof. See Appendix D.

In short, Table 2 shows the closed-form equations to obtain the optimal values of the decision variables in the cooperative supply chain, as well as the ones obtained using the Nash and the Stackelberg games.

\subsection{The coordination contract}

In non-cooperative form, a cost-sharing (CS) contract is applied in both Nash and Stackelberg games to improve the coordination between the manufacturer and the distributor. The profit functions of the members are formulated and the closed-form equations for the optimal decision variables using Nash and Stackelberg games in the distributor's dominant case are obtained.

\section{(c) CS contract under Nash game}

In the CS contract, a percentage $(0<\Delta<1)$ of the required cost to reduce the carbon emission rate is paid by the distributor and the rest, $(1-\Delta) \%$, is still the manufacturer's responsibility to pay. Similarly, $\Gamma \%$ of the investment required to provide high-quality products is provided by the distributor and the rest by the manufacturer. As such, the manufacturer's and the distributor's profit functions will change as shown in equations (4.20) and (4.21), respectively.

$$
\begin{aligned}
\Pi_{\mathrm{M}}= & \left(w-c_{m}\right)(O-Q(r, q))+\left(w-c_{m}+T-r\right) Q(r, q)-(1-\Delta) \phi \frac{s^{2}}{2} \\
& -(1-\Gamma) \theta q^{2}-p_{s}\left[\lambda(1-s) O-L_{s}\right] \\
E\left(\prod_{\mathrm{R}}(p, I)\right)= & {\left[\left(I+\beta_{0}-\beta_{1} p+\beta_{2} s+\beta_{3} r+\beta_{4} q\right)(p-w)\right]+h^{-}(I-\mu) } \\
& -\Delta \phi \frac{s^{2}}{2}-\Gamma \theta q^{2}-\left(p+h^{+}+h^{-}\right) \int_{0}^{I} F(\varepsilon) \mathrm{d} \varepsilon .
\end{aligned}
$$

Similar to the Nash game, the manufacturer and the distributor assign their optimal decisions simultaneously. 
Theorem 4.6. The optimal values of the manufacturer's decision variables in the CS contract under Nash game are obtained in equations (4.23) to (4.26).

$$
\begin{aligned}
q^{\mathrm{N}-\mathrm{CS}} & =\frac{B_{15}^{\prime} w^{\mathrm{N}-\mathrm{CS}}+B_{16}^{\prime} s^{\mathrm{N}-\mathrm{CS}}+B_{17}^{\prime}}{2(1-\Gamma) \theta} \\
r^{\mathrm{N}-\mathrm{CS}} & =\frac{-B_{11}^{\prime} w^{\mathrm{N}-\mathrm{CS}}-B_{12}^{\prime} s^{\mathrm{N}-\mathrm{CS}}+B_{14}^{\prime}}{B_{13}^{\prime}} \\
s^{\mathrm{N}-\mathrm{CS}} & =\frac{B_{25}^{\prime} m^{\mathrm{N}-\mathrm{CS}}-B_{26}^{\prime} I^{\mathrm{N}-\mathrm{CS}}-B_{27}^{\prime}}{B_{24}^{\prime}} \\
w^{\mathrm{N}-\mathrm{CS}} & =\frac{B_{28}^{\prime} m^{\mathrm{N}-\mathrm{CS}}-B_{29}^{\prime} I^{\mathrm{N}-\mathrm{CS}}-B_{30}^{\prime}}{B_{21}^{\prime}} .
\end{aligned}
$$

Proof. See Appendix E.

Meanwhile, the optimal variables of the distributor derived from equation (4.22) presented in the following theorem.

Theorem 4.7. The optimal values of the distributor's decision variables in the CS-Contract under Nash game are:

$$
\begin{aligned}
I^{\mathrm{N}-\mathrm{CS}} & =\frac{1+2 E B_{32}^{\prime} B_{34}^{\prime}+2 E B_{31}^{\prime} B_{35}^{\prime}}{3 B_{34}^{\prime}}+\frac{F_{1}^{\prime}-F_{2}^{\prime}}{F_{7}^{\prime}}+F_{7}^{\prime} \\
m^{\mathrm{N}-\mathrm{CS}} & =\frac{-2 E B_{33}^{\prime}-2 E B_{32}^{\prime} I^{\mathrm{N}-\mathrm{CS}}+\left(I^{\mathrm{N}-\mathrm{CS}}\right)^{2}}{2 E B_{31}^{\prime}}
\end{aligned}
$$

Proof. See Appendix F.

\section{(d) CS contract under Stackelberg game}

In the CS contract under a Stackelberg game, the distributor is a leader and the manufacturer is a follower. Therefore, matching the backward solution in Stackelberg game, firstly, the optimum values of the manufacturer's profit function (4.21) are assigned. Then, the derived variables of the manufacturer will be replaced into the distributor's profit function (4.22) to obtain the distributor's optimal variables. The following two theorems present the closed-form equations to obtain the decision variables.

Theorem 4.8. The optimal values of the manufacturer's decision variables when the CS-contract case is used in the Stackelberg game with the distributor playing the dominant role are:

$$
\begin{aligned}
q^{\mathrm{SR}-\mathrm{CS}} & =\frac{B_{15}^{\prime} w^{\mathrm{SR}-\mathrm{CS}}+B_{16}^{\prime} s^{\mathrm{SR}-\mathrm{CS}}+B_{17}^{\prime}}{2(1-\Gamma) \theta} \\
r^{\mathrm{SR}-\mathrm{CS}} & =\frac{-B_{11}^{\prime} w^{\mathrm{SR}-\mathrm{CS}}-B_{12}^{\prime} s^{\mathrm{SR}-\mathrm{CS}}+B_{14}^{\prime}}{B_{13}^{\prime}} \\
s^{\mathrm{SR}-\mathrm{CS}} & =\frac{B_{25}^{\prime} m^{\mathrm{SR}-\mathrm{CS}}-B_{26}^{\prime} I^{\mathrm{SR}-C S}-B_{27}^{\prime}}{B_{24}^{\prime}} \\
w^{\mathrm{SR}-\mathrm{CS}} & =\frac{B_{28}^{\prime} m^{\mathrm{SR}-\mathrm{CS}}-B_{29}^{\prime} I^{\mathrm{SR}-\mathrm{CS}}-B_{30}^{\prime}}{B_{21}^{\prime}} .
\end{aligned}
$$

Proof of Theorem 4.8 is the same as the one given for Theorem 4.6 and is presented in Appendix E. 
TABLE 3. Closed-form equations to obtain the decision variables when the CS contract is used.

\begin{tabular}{|c|c|c|}
\hline $\begin{array}{l}\text { Decision } \\
\text { vari- } \\
\text { ables }\end{array}$ & CS-Nash & CS-Stackelberg \\
\hline$(s)$ & $\frac{B_{25}^{\prime} m^{\mathrm{N}-\mathrm{CS}}-B_{26}^{\prime} I^{\mathrm{N}-\mathrm{CS}}-B_{27}^{\prime}}{B_{24}^{\prime}}$ & $\frac{B_{25}^{\prime} m^{\mathrm{SR}-\mathrm{CS}}-B_{26}^{\prime} I^{\mathrm{SR}-\mathrm{CS}}-B_{27}^{\prime}}{B_{24}^{\prime}}$ \\
\hline$(r)$ & $\frac{-B_{11}^{\prime} w^{\mathrm{N}-\mathrm{CS}}-B_{12}^{\prime} s^{\mathrm{N}-\mathrm{CS}}+B_{14}^{\prime}}{B_{13}^{\prime}}$ & $\frac{-B_{11}^{\prime} w^{\mathrm{SR}-\mathrm{CS}}-B_{12}^{\prime} s^{\mathrm{SR}-\mathrm{CS}}+B_{14}^{\prime}}{B_{13}^{\prime}}$ \\
\hline$(q)$ & $\frac{B_{15}^{\prime} w^{\mathrm{N}-\mathrm{CS}}+B_{16}^{\prime} s^{\mathrm{N}-C S}+B_{17}^{\prime}}{2(1-\Gamma) \theta}$ & $\frac{B_{15}^{\prime} w^{\mathrm{SR}-\mathrm{CS}}+B_{16}^{\prime} s^{\mathrm{SR}-\mathrm{CS}}+B_{17}^{\prime}}{2(1-\Gamma) \theta}$ \\
\hline$(w)$ & $\frac{B_{28}^{\prime} m^{\mathrm{N}-\mathrm{CS}}-B_{29}^{\prime} I^{\mathrm{N}-\mathrm{CS}}-B_{30}^{\prime}}{B_{21}^{\prime}}$ & $\frac{B_{28}^{\prime} m^{\mathrm{SR}-\mathrm{CS}}-B_{29}^{\prime} I^{\mathrm{SR}-\mathrm{CS}}-B_{30}^{\prime}}{B_{21}^{\prime}}$ \\
\hline$(m)$ & $\frac{-2 E B_{33}^{\prime}-2 E B_{32}^{\prime} I^{\mathrm{N}-\mathrm{CS}}+\left(I^{\mathrm{N}-\mathrm{CS}}\right)^{2}}{2 E B_{31}^{\prime}}$ & $\frac{-S_{20}^{\prime}-S_{18}^{\prime} I^{\mathrm{SR}-\mathrm{CS}}+S_{19}^{\prime}\left(I^{\mathrm{SR}-\mathrm{CS}}\right)^{2}}{S_{17}^{\prime}}$ \\
\hline$(I)$ & $\frac{1+2 E B_{32}^{\prime} B_{34}^{\prime}+2 E B_{31}^{\prime} B_{31}^{\prime}}{3 B_{34}^{\prime}}+\frac{F_{1}^{\prime}-F_{2}^{\prime}}{F_{7}^{\prime}}+F_{7}^{\prime}$ & $\frac{S_{14}^{\prime} S_{17}^{\prime}+S_{15}^{\prime} S_{18}^{\prime}+S_{12}^{\prime} S_{19}^{\prime}}{3 S_{15}^{\prime} S_{19}^{\prime}}+\frac{G_{1}+G_{2}}{G_{7}}-G_{7}$ \\
\hline
\end{tabular}

Afterward, in order to determine the optimum decision variables of the distributor, equations (4.29) to (4.32) are substituted in distributor's profit function (4.22), which rearranges the distributor's profit to:

$$
\prod_{\mathrm{R}}^{\mathrm{CS}-\mathrm{SR}}=\left(S_{1}^{\prime} m+S_{2}^{\prime} I+S_{3}^{\prime}\right) m+h^{-}(I-\mu)-\left(S_{4}^{\prime} m-S_{5}^{\prime} I+S_{6}^{\prime}\right) \frac{I^{2}}{2 E}+S_{7}^{\prime} I+S_{8}^{\prime} m+S_{9}^{\prime} I^{2}+S_{10}^{\prime} m^{2}
$$

where the constant parameters $S_{1}^{\prime}$ to $S_{10}^{\prime}$ are presented in Appendix G.

Theorem 4.9. The closed-form equations for the optimal values of the distributor's decision variables are shown in equations (4.34) and (4.35).

$$
\begin{aligned}
I^{\mathrm{SR}-\mathrm{CS}} & =\frac{G_{1}+G_{2}}{G_{7}}-G_{7}+\frac{S_{14}^{\prime} S_{17}^{\prime}+S_{15}^{\prime} S_{18}^{\prime}+S_{12}^{\prime} S_{19}^{\prime}}{3 S_{15}^{\prime} S_{19}^{\prime}} \\
m^{\mathrm{SR}-\mathrm{CS}} & =\frac{-S_{20}^{\prime}-S_{18}^{\prime} I^{\mathrm{SR}-\mathrm{CS}}+S_{19}^{\prime}\left(I^{\mathrm{SR}-\mathrm{CS}}\right)^{2}}{S_{17}^{\prime}} .
\end{aligned}
$$

Proof. See Appendix G.

Table 3 contains closed-form equations to obtain the optimal values of the decision variables when the CS contract is used in both the Nash and the Stackelberg games.

\section{A PRACTICAL CASE AND THE COMPUTATIONAL RESUltS}

In order to study the applicability of this research and analyze the trend of the optimal decision variables provided in the theorems, a real case of the car tire industry is considered. The tire industry is one of the lucrative productions in the United States with annual revenue of $\$ 17.6$ billion, which indicates the important role of car tire in today's competitive market [7]. One report published by the Center for Remanufacturing and Re-used in the UK presents that using new approaches in production's flow of tires releases the amount of emission. The change of casing in the tire production process has resulted in releasing $60.5 \mathrm{KG}$ carbon as compared to the traditional tire which releases $86.9 \mathrm{KG}$ carbon [13]. Moreover, the car tire remanufacturing is one of the largest sectors of remanufacturing industry in the US, because the retread of tires yields to materials and energy saving in which the costs of remanufacturing is 30\%-50\% less than the production of a new tire [7]. The benefits of the tire remanufacturing persuade reputable companies in the tire industry such as Goodyear, 
Bridgestone Bandag, and Michelin, which share $\$ 686.4$ million of the industrial revenue [51], to take part in the remanufacturing. As California is one of the biggest economies and one of the largest pollution emitters in the world, California's Global Warming Solutions Act of 2006 (AB32) implements various policies under the cap and trade regulation to obtain the emission to 1990 levels by 2020. In order to emphasize the effects of the cap and trade regulation in practice, they presented and discussed the tire industry [86]. In addition, the quality level of products is a motivating factor in the tire industry. The tire production based on customer satisfaction like providing non-slip tires, non-explosive forbiddance or no bulgy can enhance the market's share [26].

Here, the database of an Iranian tire supply chain is provided to analyze the efficiency of using a CS contract in presence of quality improvement efforts, carbon abatement possibilities, pricing strategy, returning and remanufacturing, and also to study the impacts of a CS contract on the aforementioned motivating factors. The values of the parameters used in this research are achieved by the help of experts and consultants in the Iranian tire supply chain. In addition, regarding the value of the parameter $\beta_{1}$, a marketing approach is needed. Audits of the Iranian tire supply chain should collect data that connect price of tires and demand of the market. Then, a regression analysis between the price and demand, which are denoted as independent and dependent variables respectively, leads to determination of price sensitivity. Furthermore, the value of the parameter $\beta_{2}$ is a marketing output as well. Experts should record database which links the amount of carbon released under each unit of tire production and demand of market. A regression analysis between each unit of tire carbon emission and demand of market reveals the value of $\beta_{2}$. Carbon emission data can be found in previous projects implemented in the Iranian tire supply chain in terms of carbon abatement. In order to obtain the value of parameter $\beta_{3}$, similar to determination of the price sensitivity $\left(\beta_{1}\right)$ and the carbon abatement coefficient $\left(\beta_{2}\right)$, the dataset which connect the refund price paid by the manufacturer to the customers for collecting the defected products and the market demand can lead to measuring $\beta_{3}$. It happens by regression analysis between the refund price and the market demand. The values of the other parameters including $\beta_{4}, \delta$, and $\gamma$ are determined similar to the approach proposed for the price or carbon coefficient impacts.

Moreover, there is an institution that subcontracts the Iranian government, namely IEEO $^{1}$, founded in 1960s, which is responsible to control the carbon emission under production in all types of industries. Therefore, the value of the parameters used in the trade and cap regulation, i.e. $L_{s}$ and $p_{s}$, are determined by the IEEO experts.

Other parameters used in this research such as the unit production cost for new and remanufactured products, $C_{m}, C_{r}$, the unit saving cost, $T$, the investment coefficient of carbon abatement and the quality improvement, $\theta, \phi$, and the carbon discharging rate of each production unit, $\lambda$, are determined using a questionnaire of the commissioners and specialists in the Iranian tire production supply chain.

The measured values of the selected parameters to investigate the sensitivity of the decision variables and the supply chain profit on the values of some selected parameters in both cooperative and non-cooperative forms are $C_{m}=\$ 7, C_{r}=\$ 5, h^{-}=0.8$ (dollars/tires), $h^{+}=0.6$ (dollars/tires), $\lambda=1.25$ (grams/dollars), $\beta_{0}=800$ (tires), $\beta_{1}=4$ (tires/dollars), $\beta_{2}=0.9$ (dollars), $\beta_{3}=6$ (tires/dollars), $\beta_{4}=7$ (tires), $\alpha=70$ (tires), $\delta=3$ (tires/dollars), $\gamma=0.6$ (tires), $p_{s}=1.5$ (dollars $/$ grams), $L_{s}=74$ (grams), $E=80$ (tires), $\phi=\$ 3800, \quad \theta=\$ 700$, and $\mu=40$ (tires).

In the cooperative case, the optimal decision variables and the total profit of the supply chain are obtained as $S^{\mathrm{C}}=0.96, r^{\mathrm{C}}=\$ 449.5, p^{\mathrm{C}}=\$ 465.5, q^{\mathrm{C}}=2.48, I^{\mathrm{C}}=79.7$ (tires), and $\prod^{\mathrm{C}}=\$ 140099.5$. For the noncooperative case when the Nash game is used we obtain $w^{\mathrm{N}}=\$ 135.7, S^{\mathrm{N}}=0.28, r^{\mathrm{N}}=\$ 118.2, p^{\mathrm{N}}=\$ 260.9$, $m^{\mathrm{N}}=\$ 125.17, q^{\mathrm{N}}=0.68, I^{\mathrm{N}}=38.4$ (tires), $\prod_{\mathrm{R}}=\$ 61365.7, \prod_{\mathrm{M}}=\$ 16456.7$, and $\prod_{S}=\$ 77822.4$. In the Stackelberg case, the optimal values of the decision variables and the profits of the chain and its members become $w^{\mathrm{SR}}=\$ 199.1, S^{\mathrm{SR}}=0.42, r^{\mathrm{SR}}=\$ 181.9, p^{\mathrm{SR}}=\$ 292.7, m=\$ 93.6, q=1.03, I=35.9$ (tires), $\prod_{\mathrm{R}}=\$ 69210.3, \prod_{\mathrm{M}}=\$ 36111.8$, and $\prod_{S}=\$ 105322.1$. The above results illustrate that the profit of the SC in the cooperative case is higher than the ones in the Nash and Stackelberg games. This is an indication of the important role of applying a cooperative form as a solution to improve the supply chain's profit. In addition,

\footnotetext{
${ }^{1}$ Iran Energy Efficiency Organization.
} 
the quality effort $(q)$ and the carbon emission reduction $(s)$ in the cooperative form are higher than the ones in the non-cooperative form. It means that under cooperation, the manufacturer is more eager to invest in the improvement of the quality and reduction of carbon emission, leading to the higher demand of the market and hence higher profit of the supply chain, the manufacturer, and the distributor. Moreover, in the non-cooperative form, the chain profit when the Stackelberg game is employed is higher than the one in the Nash game. This is due to different decision sequences obtained in these two games, which leads to higher rates of variables $s$ and $q$ in the Stackelberg game.

\subsection{The effects of parameter changes in the cooperative case}

Figures $2 \mathrm{a}$ and $2 \mathrm{~b}$ illustrate the impacts of the parameter $\beta_{2}$ in the interval $[0.1,1.2]$ on the decision variables in the cooperative form. The increase of carbon sensitivity leads to a drop in the final price $(p)$ and a spike in carbon abatement rate $(s)$. The carbon reduction motivates customers to purchase more products and enhances market demand. There may be a point at issue that the decrement of the final price reduces cooperative supply chain. However, the benefits of the demand increment suppress the price decrement. As a result, the cooperative supply chain becomes more affordable to invest in motivating factors, where the carbon abatement rate $(s)$ and the quality effort $(q)$ increases (see Figs. 2a and 2b). Therefore, a shrink in the price and a spike in the carbon reduction and the quality improvement effort motivate the customers' willingness to buy the products more, and consequently, to increase the profit of cooperative supply chain (see Fig. 2c). These results show the efficiency of the cooperative chain, which not only increases the profit of the chain but also encourage the manufacturer to produce more environmentally friendly and high-quality products via lower price.

The given Figures $2 \mathrm{~d}$ and $2 \mathrm{e}$ indicate the effects of the parameter $\beta_{3}$ on the decision variables in the cooperative case. As seen in these figures, an increase of the parameter $\beta_{3}$ in the interval $[0.5,6]$ leads to an increase in the refund price $(r)$ and the final price $(p)$. Note that the higher the refund price is the higher the quantity of the returns would be. This leads to remanufacture more returns. Remanufacturing has environmental benefits, and consequently, the carbon reduction rate $(s)$ rises up (shown in Fig. 2e). Moreover, remanufacturing not only can reduce the carbon emission, but also can improve the quality of the products. It may be noted that although the price of products increases, the increase of variables $s, r$ and $q$ can motivate the customers to purchase the products. Therefore, the market's demand increases, and consequently, the supply chain's profit (matching to Fig. 2e) rises up. These results confirm the importance of remanufacturing activities in supply chains under the cooperative form.

Figures $2 \mathrm{f}$ and $2 \mathrm{~g}$ illustrate the effects of the parameter $\beta_{4}$ on the decision variables. As shown in these figures, an increase of $\beta_{4}$ in the interval $[1,10]$ raises the quality effort $(q)$ and the final price of the product $(p)$. It should be noted that a higher quality level of products leads to a higher refund price $(r)$. Therefore, the refund price $(r)$ increases, which yields an increase in the quantity of the returned products. As a result, remanufacturing of the returns improves, which reduce the carbon emission. Therefore, the carbon reduction rate, according to Figure 2f, increases. Although the price of products rises up, the increase of variables $s, q$ and $r$ motivate the customer's willingness to buy products more. Therefore, the market's demand improves, which leads to an increase in the supply chain's profit (shown in Fig. 2g). These results present the significant role of investment in quality improvement, which has environmental and economic benefits under the cooperative supply chain.

\subsection{Analysis of the CS-contract in the Nash game}

The given Figures 3a and 3b show the effects of an increase of the parameter $\Delta$ on the values of decision variables in which $\Gamma$ is fixed $(\Gamma=0.4)$. These figures demonstrate that an increase of $\Delta$ raises the carbon reduction rate $(s)$, but none of the final price $(p)$, the wholesale price $(w)$, and the refund price $(r)$ are sensitive to $\Delta$. An increase of $\Delta$ by the distributor persuades the manufacturer to invest more in low-carbon production, and as a result, the variable $s$ rises up. In addition, the reduction of carbon emission encourages customers to buy the product more. Therefore, the distributor's profit increases when $\Delta \leq 0.45$ according to Figure 3c. 


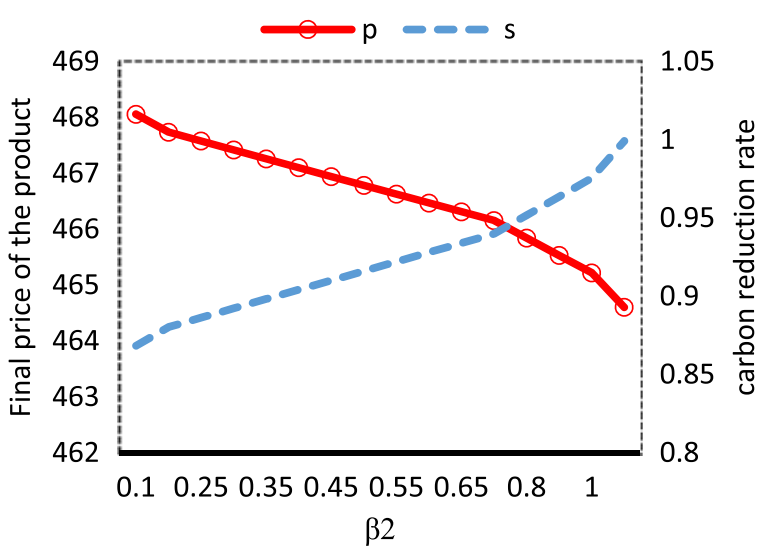

(a)

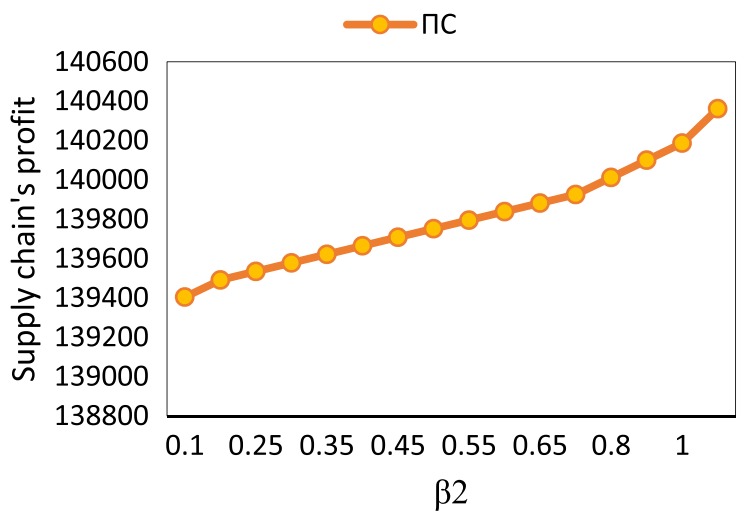

(c)

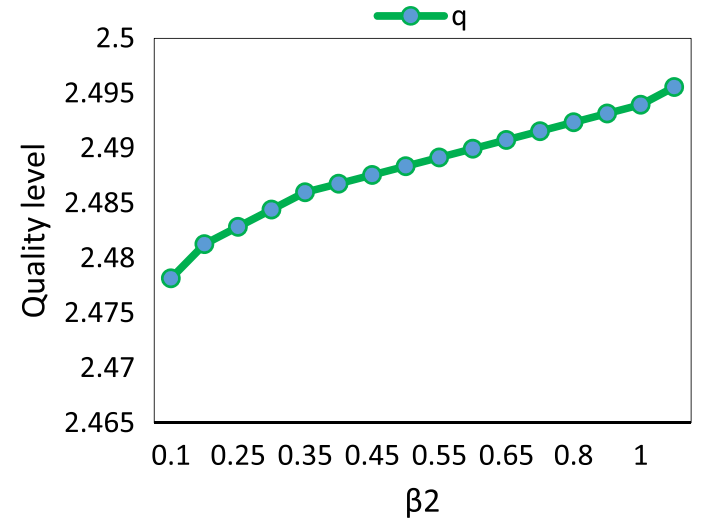

(b)

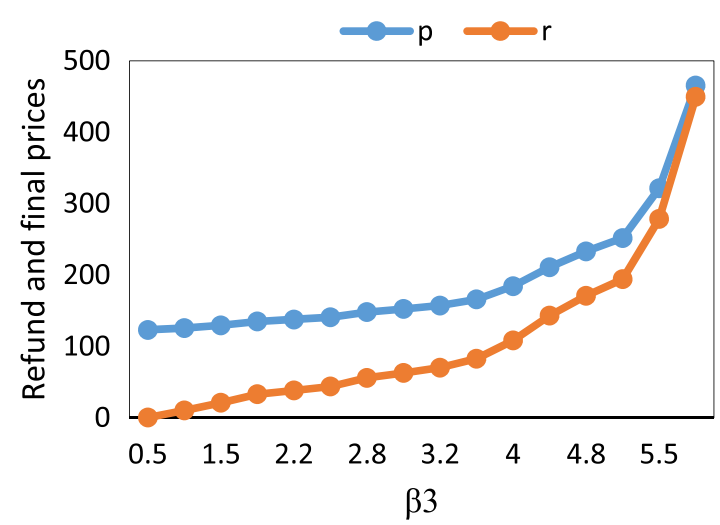

(d)

Figure 2. (a) The carbon reduction rate and the final price under levels of $\beta_{2}$ in cooperative case. (b) The quality effort under levels of $\beta_{2}$ in cooperative case. (c) The supply chain's profit under levels of $\beta_{2}$ in cooperative case. (d) The refund price and the final price under levels of $\beta_{3}$ in cooperative case. (e) the carbon rate, the quality effort and the supply chain's profit under levels of $\beta_{3}$ in cooperative case. (f) Quality effort and carbon reduction under levels of $\beta_{4}$ in cooperative case. (g) The refund price, the final price and supply chain's profit under levels of $\beta_{4}$ in cooperative case.

However, when $\Delta>0.45$, his/her profit drops off. This is due to the fact that the sharing cost undertaken by the distributor is higher than the benefits of the demand's enhancement and hence undertaking the rate of $\Delta$ more than 0.45 is not acceptable for the distributor. On the other hand, although the manufacturer's profit decreases under the CS contract in the Nash game (shown in Fig. 3c), his/her profit under each rate of $\Delta$ is higher than the profit without contract $\left(\prod_{\mathrm{M}}^{\mathrm{N}}=16456.7\right)$. Therefore, according to Figure 3d, the supply chain's profit using the CS contract in $\Delta \leq 0.4$ increases, but decreases for $\Delta>0.4$. Therefore, the optimal rate to maximize the supply chain's profit in the CS contract under the Nash game is $\Delta=0.4$. It should be pointed out that under each rate of $\Delta$, the supply chain's profit is higher than the profit without the contract $\left(\prod_{S}^{\mathrm{N}}=77822.4\right)$, which shows the benefit of using a CS contract under the Nash game to provide better coordination in the supply chain. 


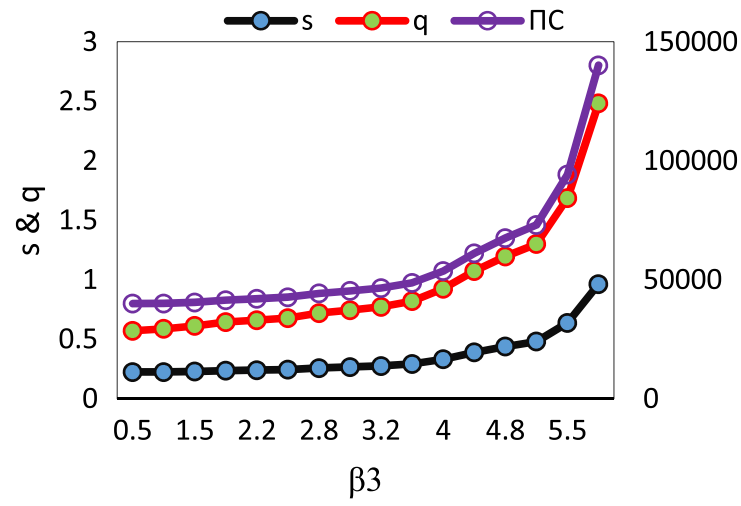

(e)

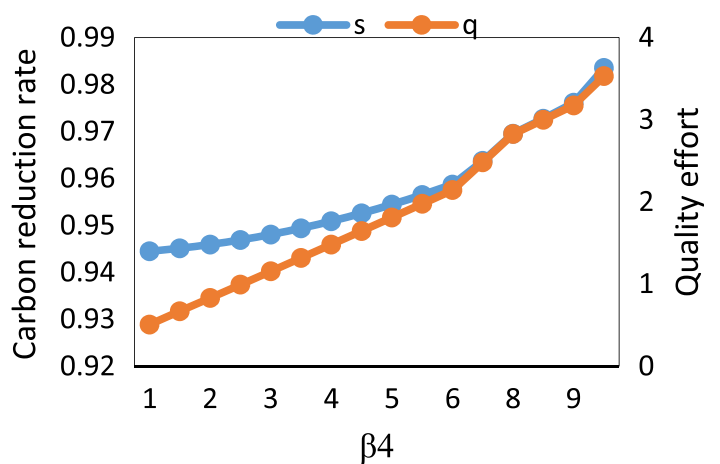

(f)

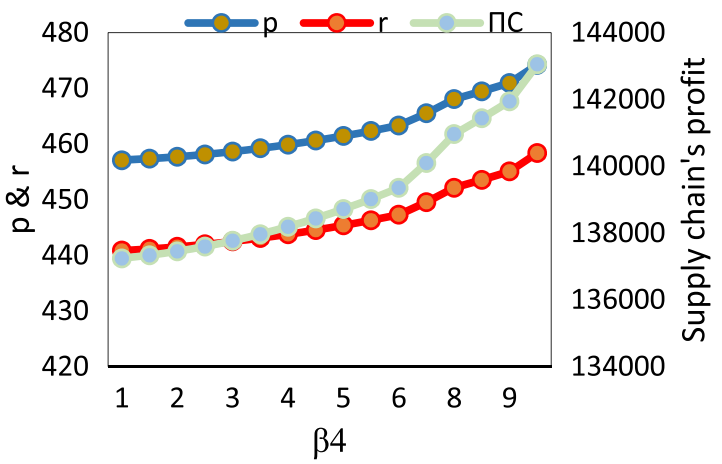

(g)

Figure 2. (Continued.)

Figures $4 \mathrm{a}$ and $4 \mathrm{~b}$ demonstrate the impacts of the parameter $\Gamma$ on the decision variables for which $\Delta$ is fixed $(\Delta=0.38)$. It can be seen from these figures that an increase of $\Gamma$ in the interval $[0.1,0.7]$ raises the quality improvement effort of the product. However, the wholesale price $(w)$, the final price $(p)$, the refund price $(r)$, and the carbon reduction rate $(s)$ are not too sensitive to the values of the parameter $\Gamma$. Here, the distributor by paying a fraction of the quality improvement cost to the manufacturer persuades him/her to produce a higher quality product. It should be noted that although the manufacturer's profit under increase of $\Gamma$ decreases (shown in Fig. 4c), the manufacturer's profit under each rate of $\Gamma$ is higher than the profit without contract $\left(\prod_{M}^{\mathrm{N}}=16456.7\right)$. Therefore, the CS contract under the Nash game is acceptable for the manufacturer. On the other hand, quality improvement efforts raise the market's demand, and as a result, the distributor's profit for $\Gamma \leq 0.45$ increases (shown in Fig. 4c). However, when $\Gamma>0.45$, then the revenues due to the demand's increase is less than the sharing costs undertaken by the distributor. Therefore, the rates more than 0.45 for $\Gamma$ is not acceptable for the distributor. Furthermore, the supply chain's profit, according to Figure 4d, under each rate of $\Gamma$ is higher than the profit without a contract $\left(\prod_{S}^{\mathrm{N}}=77822.4\right)$, which indicates that the CS contract under the Nash game to improve the quality of the product is a sufficient solution and provides better coordination. Moreover, the optimal rate of $\Gamma$ to maximize the supply chain's profit is $\Gamma=0.45$.

\subsection{Analysis of the CS-contract rates in the Stackelberg game}

The given Figures $5 \mathrm{a}$ and $5 \mathrm{~b}$ illuminate the effects of the parameter $\Delta$ in the interval $[0.14,0.5]$ on the decision variables under the Stackelberg game in which $\Gamma=0.62$. The results indicate that although an increase of $\Delta$ 


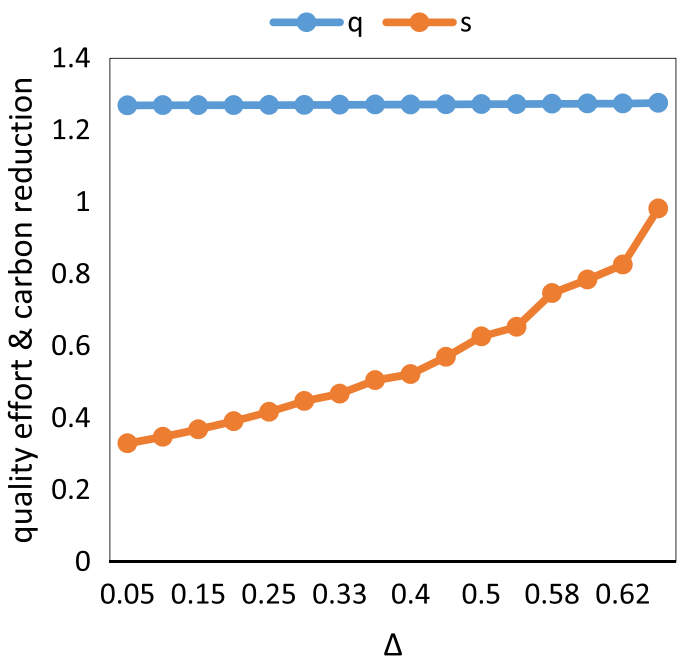

(a)

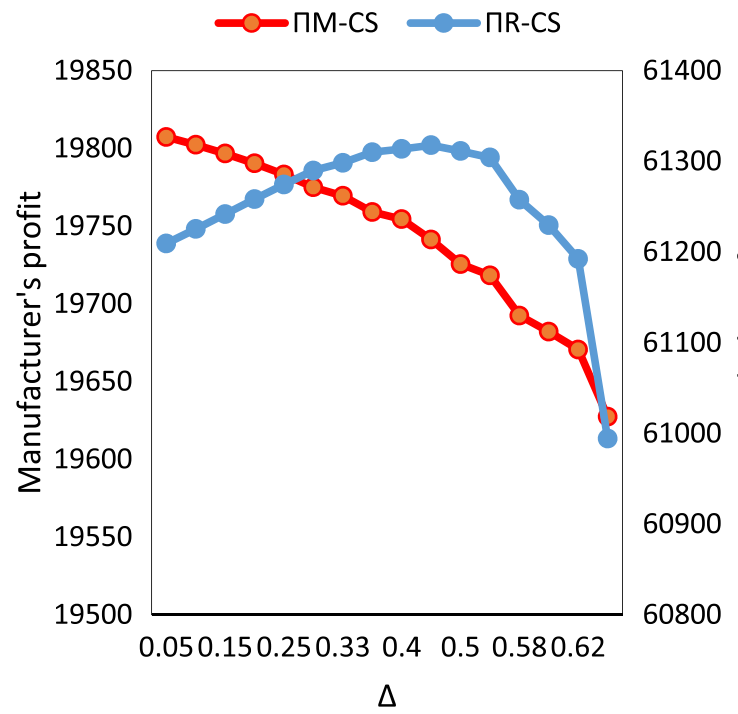

(c)

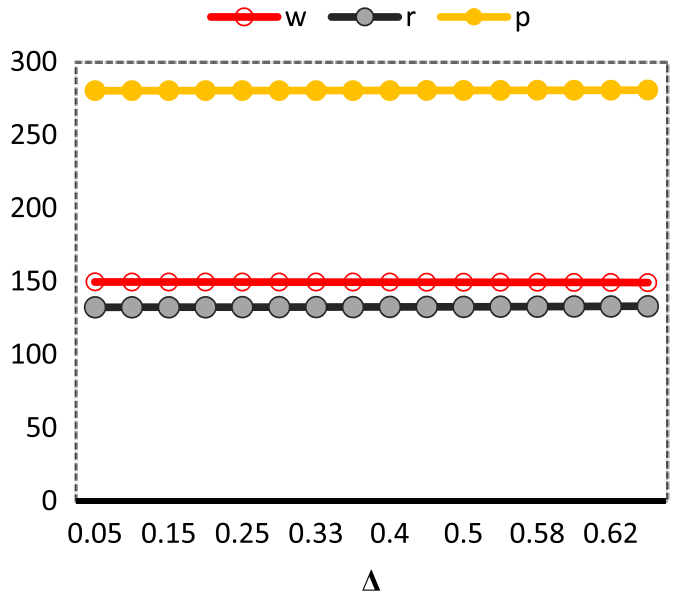

(b)

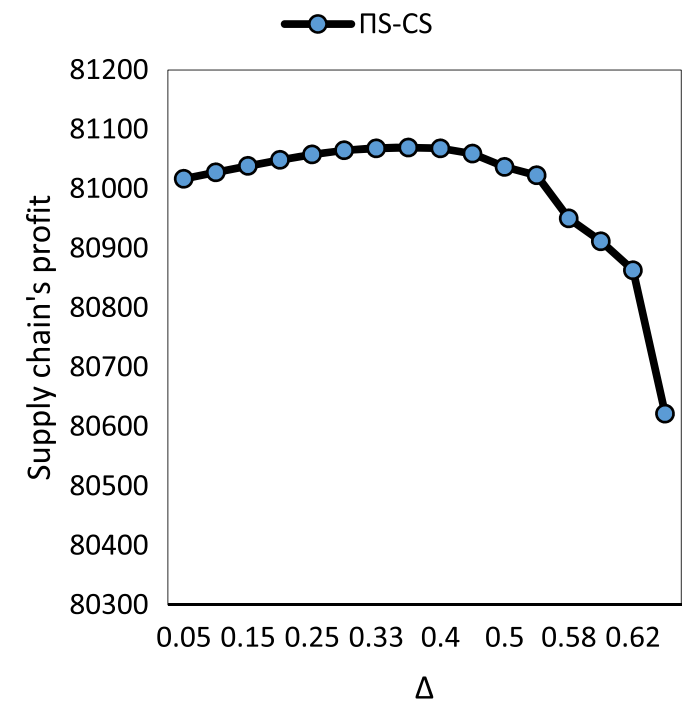

(d)

Figure 3. (a) Carbon reduction and quality effort under different levels of $\Delta$ in CS-N. (b) Wholesale, final and refund prices under different levels of $\Delta$ in CS-N. (c) Manufacturer's and distributor's profit under different levels of $\Delta$ in CS-N. (d) Supply chain's profit under different levels of $\Delta$ in CS-N.

raises the carbon reduction rate $(s)$, the quality level $(q)$ and the refund price $(r)$, the prices of the products are not sensitive to the carbon cost-sharing contract. It is because the fact that a fraction of the cost undertaken by the distributor to reduce carbon emission provides the manufacturer a better affordable condition to invest in the low-carbon products. Therefore, the variable $(s)$ improves, and consequently, the market share increases. The benefits of demand enhancement afford the manufacturer to invest in other motivating factors. As a result, 


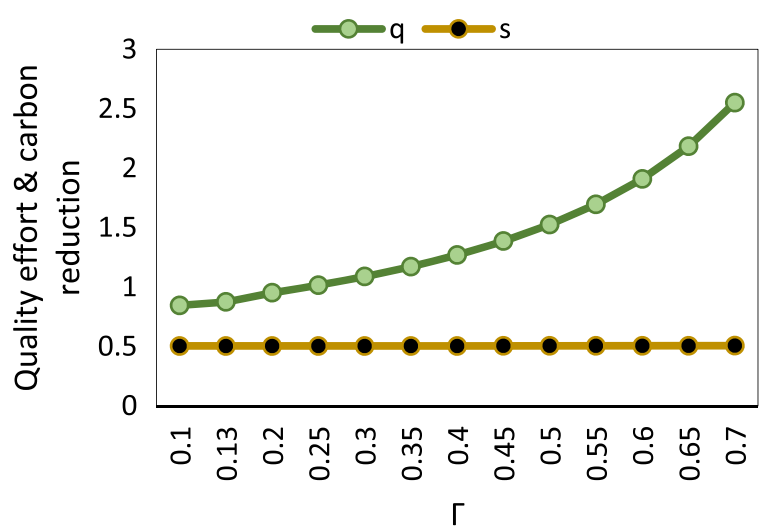

(a)

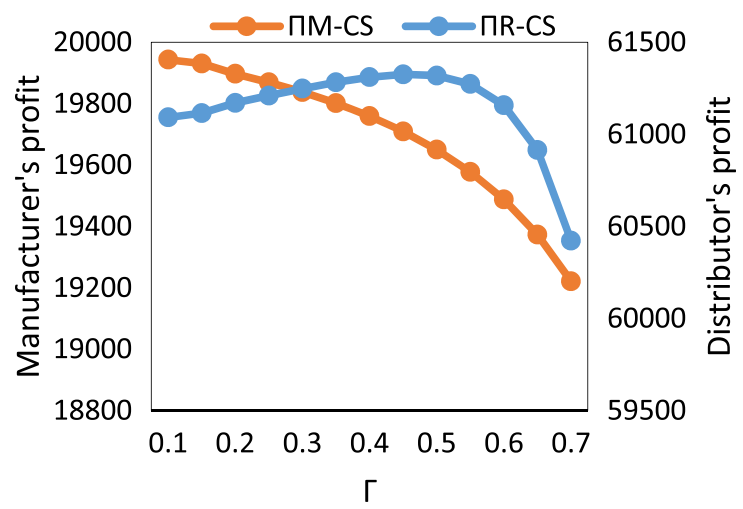

(c)

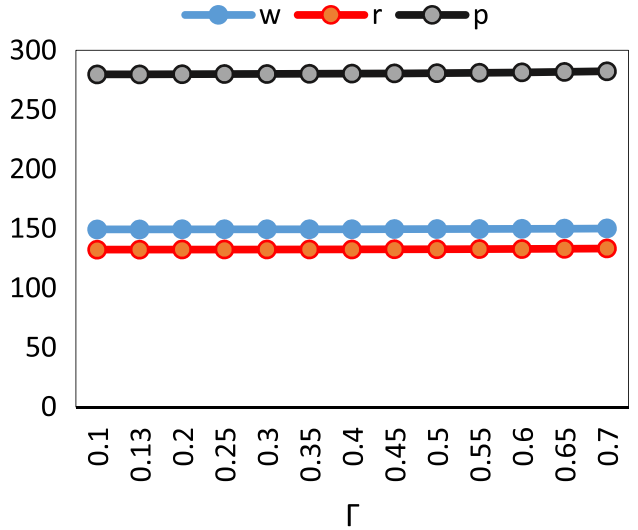

(b)

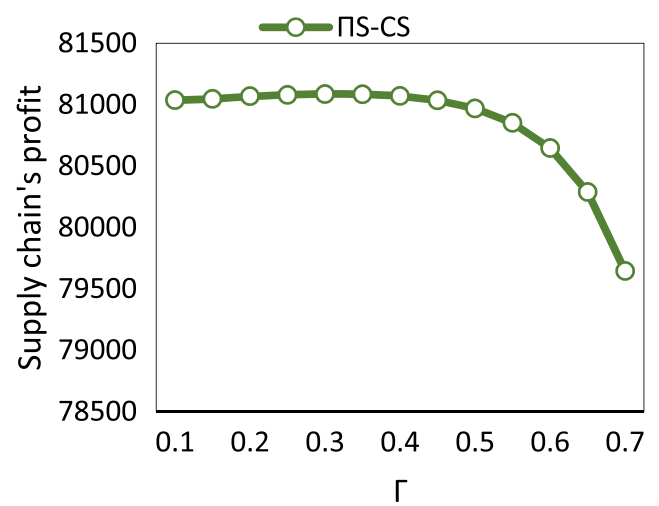

(d)

Figure 4. (a) The quality effort and the reduction rate under levels of $\Gamma$ in CS-N. (b) The wholesale price, the final price and the refund price under levels of $\Gamma$ in CS-N. (c) The distributor's and the manufacturer's profits under levels of $\Gamma$ in CS-N. (d) The supply chain's profit under levels of $\Gamma$ in $\mathrm{CS}-\mathrm{N}$.

the refund price $(r)$ and the quality effort $(q)$ increase (see Figs. 5a and 5b), which augment the market share. Therefore, the manufacturer's profit (shown in Fig. 5c) rises up, where under each rate of $\Delta$ it is higher than the profit without a contract $\left(\prod_{\mathrm{M}}^{\mathrm{SR}}=36111.8\right)$. These figures depict that using the CS contract is a good strategy for the manufacturer. On the other hand, the distributor's profit for $\Delta \leq 0.37$ increases, because in these rates the revenues obtained by an increase in the market demand can offset the sharing cost the distributor tolerates. However, the conclusion is reversed when $\Delta>0.37$ in which the distributor does not accept the higher costsharing rate of 0.37 for carbon emission reduction. Furthermore, the supply chain's profit using the CS contract under each rate of the defined $\Delta$ increases (shown in Fig. $5 \mathrm{~d}$ ), where its maximum is $\Delta=0.5$. It merits a mention that although the distributor's profit in $\Delta>0.37$ decreases, the intensity of a spike in the manufacturer's profit is more than a shrink in the distributor's profit. Therefore, the supply chain's profit increases and under each rate of $\Delta$ is higher than his/her own profit without a contract $\left(\prod_{S}^{\mathrm{SR}}=105322.1\right)$. As a result, using the CS contract in the Stackelberg game not only can boost the profit of the supply chain, but also acts as a catalyst to enhance other motivating factors, including carbon abatement, quality improvement effort, and refund price. In addition, this contract, while the sharing rate for costs of the carbon abatement increases for which the quality 


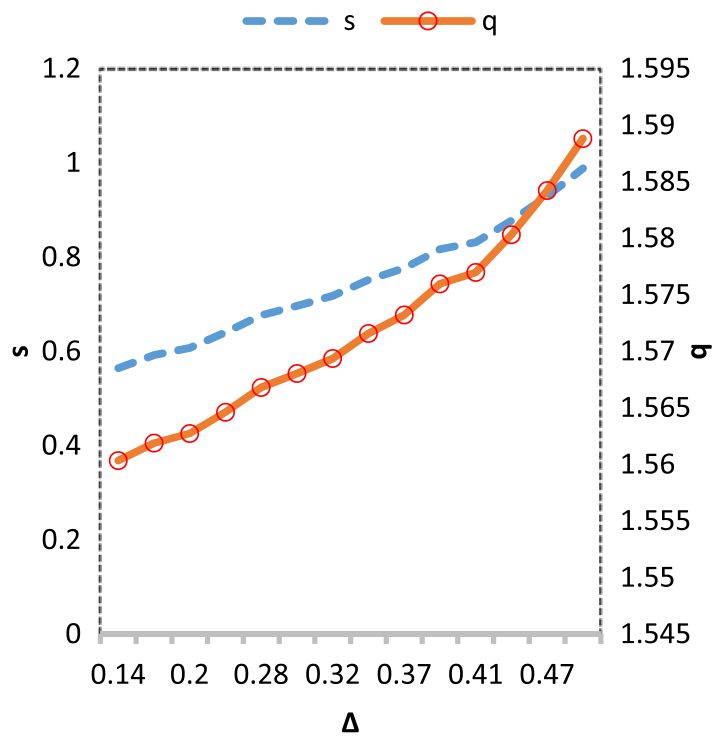

(a)

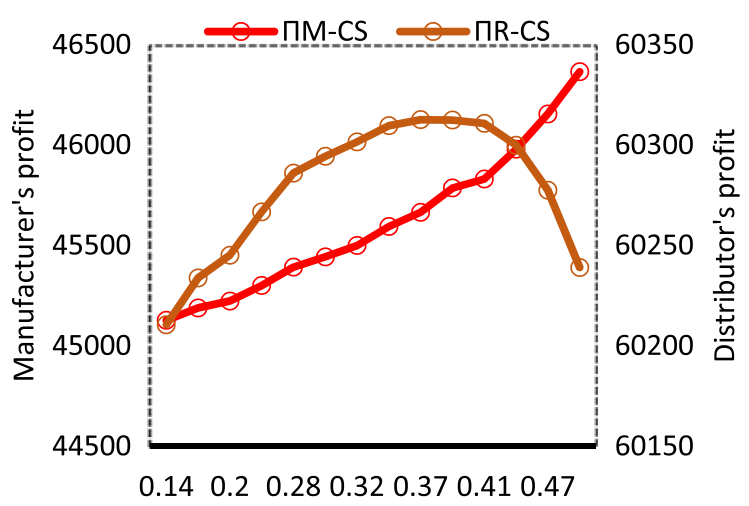

$\Delta$

(c)

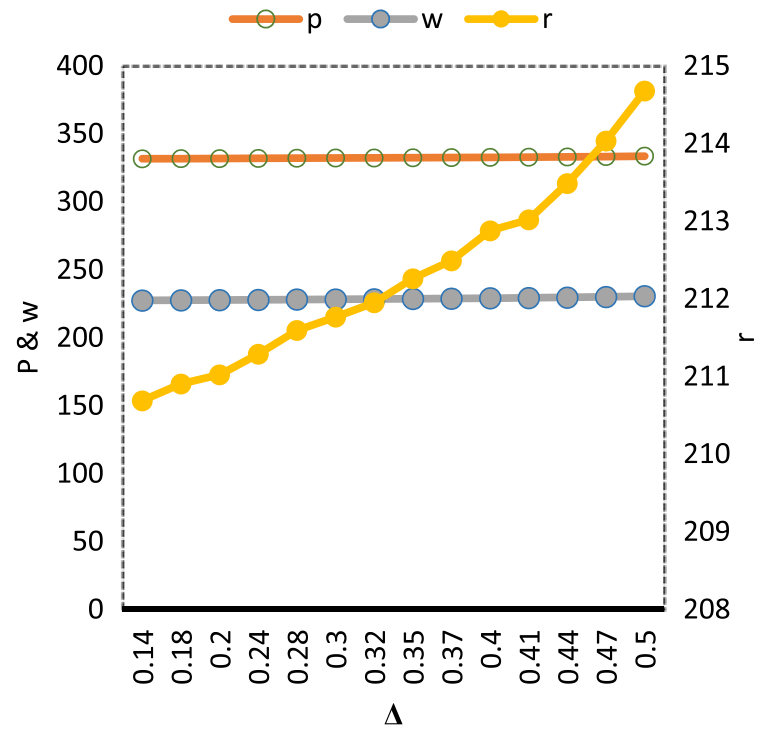

(b)

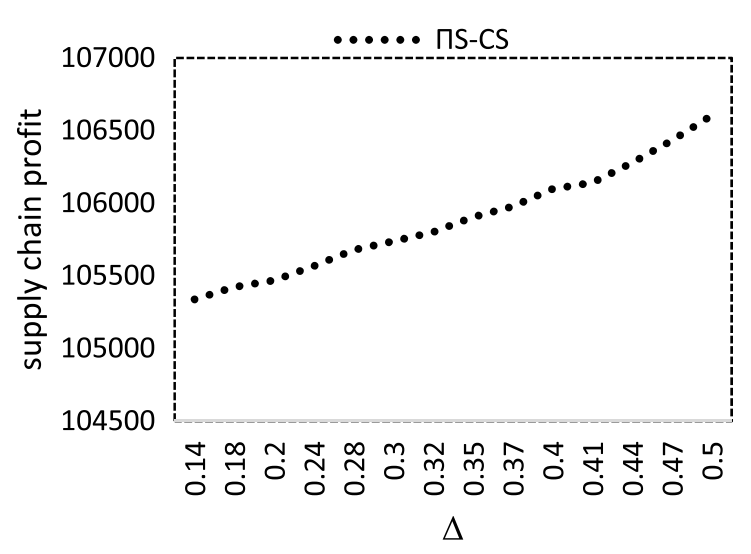

(d)

FiguRE 5. (a) The carbon reduction and the quality effort under different levels of $\Delta$ in CS-SR.

(b) The final price, the wholesale price and the refund price under levels of $\Delta$ in CS-SR.

(c) The manufacturer's and the distributor's profits under different levels of $\Delta$ in CS-SR.

(d) The supply chain's profit under different levels of $\Delta$ in CS-SR.

improvement effort is fixed, is able to hold the fixed price for products, whilst supply chain imposes more costs to enhance the applied motivating elements.

Figures $6 \mathrm{a}$ and $6 \mathrm{~b}$ present the impacts of parameter $\Gamma$ in the interval $[0.61,0.78]$ on the decision variables in which the rate of $\Gamma$ is fixed $(\Delta=0.4)$. It can be easily seen from these figures that an increase of $\Gamma$ leads to an increase of all motivating factors, including the refund price, the carbon reduction rate, and the quality improvement effort. However, it leads to an increase in the wholesale price and the final price as well. Note that although the final price increases, the improvement of the refund price, the quality effort, and the carbon reduction rate motivates the customers to purchase the product more. Therefore, a higher demand yields 


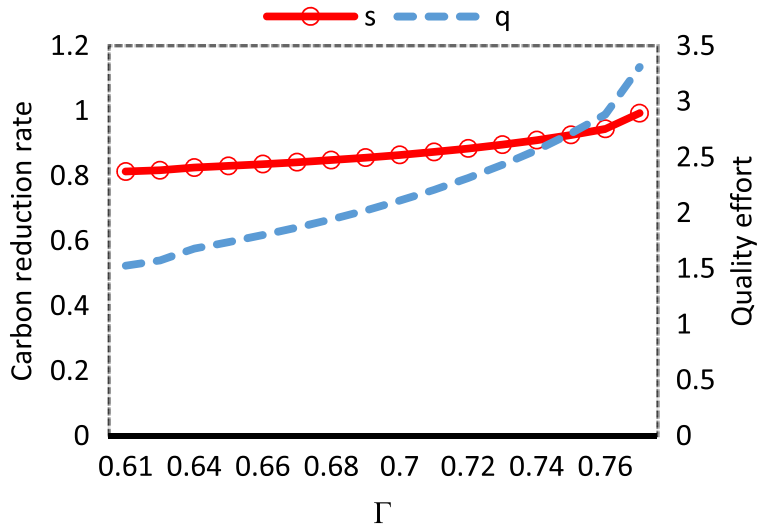

(a)

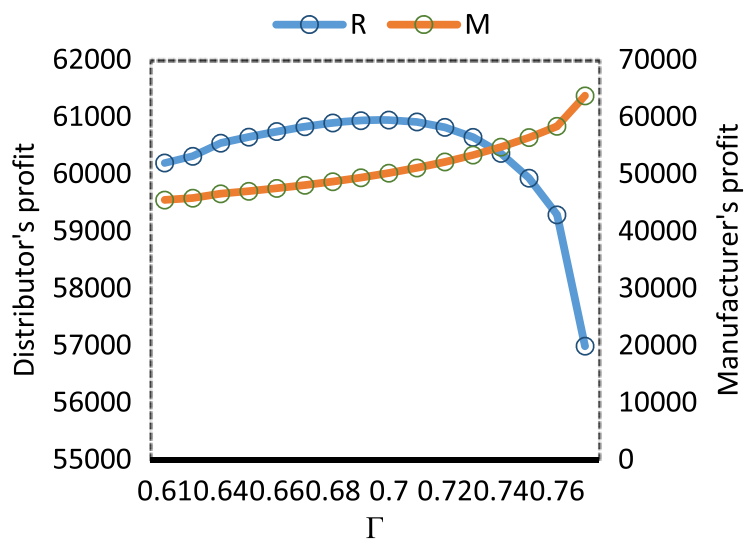

(c)

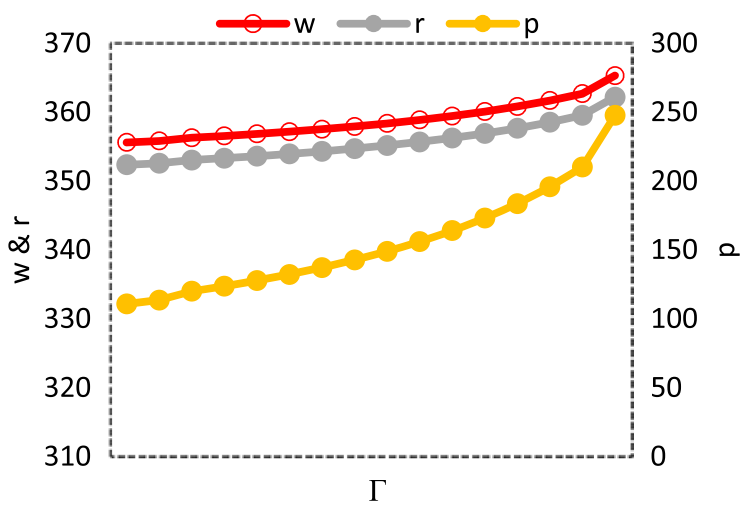

(b)

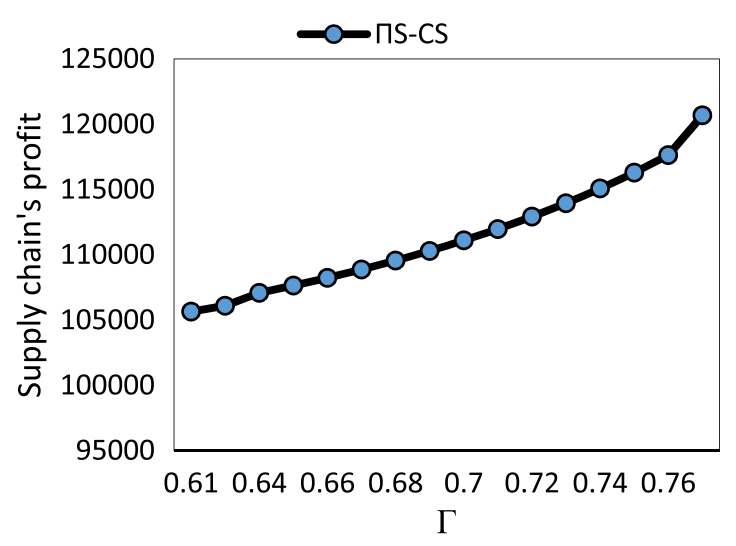

(d)

FiguRE 6. (a) The carbon reduction and the quality effort under levels of $\Gamma$ in CS-SR. (b) The final price, the refund price and the wholesale price under levels of $\Gamma$ in CS-SR. (c) The distributor's and the manufacturer's profits under levels of $\Gamma$ in CS-SR. (d) The supply chain's profit under levels of $\Gamma$ in CS-SR.

an increase of the manufacturer's profit (shown in Fig. 6c), where under each rate of $\Gamma$ the manufacturer's profit is higher than his own profit without contract $\left(\prod_{\mathrm{M}}^{\mathrm{SR}}=36111.8\right)$. Therefore, using the CS contract under the Stackelberg game is acceptable for the manufacturer. In addition, an increase in demand raises the distributor's profit in $\Gamma \leq 0.7$ (Fig. 6c). Therefore, the profit of the $\mathrm{SC}$ using the CS contract increases (according to Fig. 6d), where under each rate of the defined $\Gamma$ it is higher than the profit without the contract $\left(\prod_{S}^{\mathrm{SR}}=105322.1\right)$ where the optimal profit appears at $\Gamma=0.78$. It should be noted that for $\Gamma>0.7$, the sharing costs are more than benefits of the demand improvement, which reduces the distributor's profit. However, the profit of the SC using the CS contract even for $\Gamma>0.7$ increases (see Fig. 6d). It is due to the fact that a spike in the manufacturer's profit has more intensity rather than a drop in the distributor profit. These results reveal that in the CS contract, an increase of the cost-sharing rate for the quality improvement effort $(\Gamma)$ undertaken via the distributor is a sufficient contract to not only boost the profit of the SC but also to encourage the supply chain for high investment in other motivating factors (carbon, quality, and refund price). 
What merits mention is that there is a contrast in the analysis of the parameter $\Gamma$ rather than $\Delta$ under the Stackelberg game. For all increases of $\Delta$, although all motivating factors rise up, the final price of the product $(p)$ is still fixed. Because, when $\Delta$ increasing while $\Gamma$ is fixed, the benefits of the demand increment can overcome the costs. Thus, the supply chain does not need to raise the price. However, in case of an increase of the parameter $\Gamma$ while $\Delta$ is fixed, the results are reversed and the SC cannot afford the costs of motivating factors without increasing the final price because the benefit of the demand increase is not enough for holding the price fixed.

As we extract a plethora of valuable results in the analysis of the CS contract, Table 4 is provided in the rest of the paper to present the outcomes briefly.

\section{MAnAGERIAL insights}

According to the results achieved from the above numerical investigation, the major findings of this research which a manager can use in practice are:

- Due to the high costs of implementation in terms of quality improvement effort, carbon abatement, and collecting and remanufacturing, two solutions are assumed, including the cooperative supply chain and the CS contract in non-cooperative form. The results highlight that both approaches are sufficient and can enhance the profit of SC. Therefore, a manager in order to obtain the optimal decision variables and to maximize the profit of his company prefers to apply a CS contract or a cooperative chain.

- Analysis of the CS contract in the Nash game indicates that under this contract there is no interaction between the motivating factors. More specifically, when the distributor subcontracts the manufacturer and undertakes a higher part of the carbon abatement costs, it just sufficient to reduce carbon, but the quality level and the refund price do not react to carbon abatement. However, in the Stackelberg game, the results are reversed and an increase of carbon abatement has positive effects on the other elements such as the quality level or the refund price. Therefore, a manager having this valuable information in terms of a CS contract can choose the best strategy to maximize the benefits. If the manager desires to enhance all motivating factors simultaneously, it is better to use the Stackelberg game rather than Nash game.

- It merits a mention that in the CS contract under the Stackelberg game there is a contrast in cost-sharing rates undertaken via the distributor. When the carbon abatement sharing rate $(\Delta)$ increases while the quality improvement sharing rate $(\Gamma)$ is fixed, the motivating factors $(s, q, r)$ increases but the prices are still fixed. This boosts the affordability of customers to purchase the product more. However, when the rate of $\Gamma$ rises up while $\Delta$ is fixed, this contract cannot enhance the motivating factors without holding the prices fixed. Therefore, a manager with the knowledge of these findings should choose a strategy in which the distributor incurs a large number of the carbon abatement costs rather than quality enhancement costs. This strategy not only leads to improvement of all motivating factors but also holds the prices fixed.

- Via an analysis of the CS contract in both the Nash the Stackelberg games the optimal amount of rates in the CS contract, namely $\Delta$, and $\Gamma$, can be attained. Therefore, a manager having the optimal rates can implement the contract and boost the benefit of the SC and its members. In addition, we succeeded to obtain closed forms of the optimal decision variables, including the refund price, inventory level, quality level, carbon abatement rate, via game theoretical approaches. This helps the manager to maximize the profit of his own company by selecting the optimal decision variables.

\section{Conclusion}

Reduction of the carbon emission, improvement of the product quality, and setting a suitable pricing strategy was considered in this paper in order to attract customer's willingness to purchase the product more. Three 


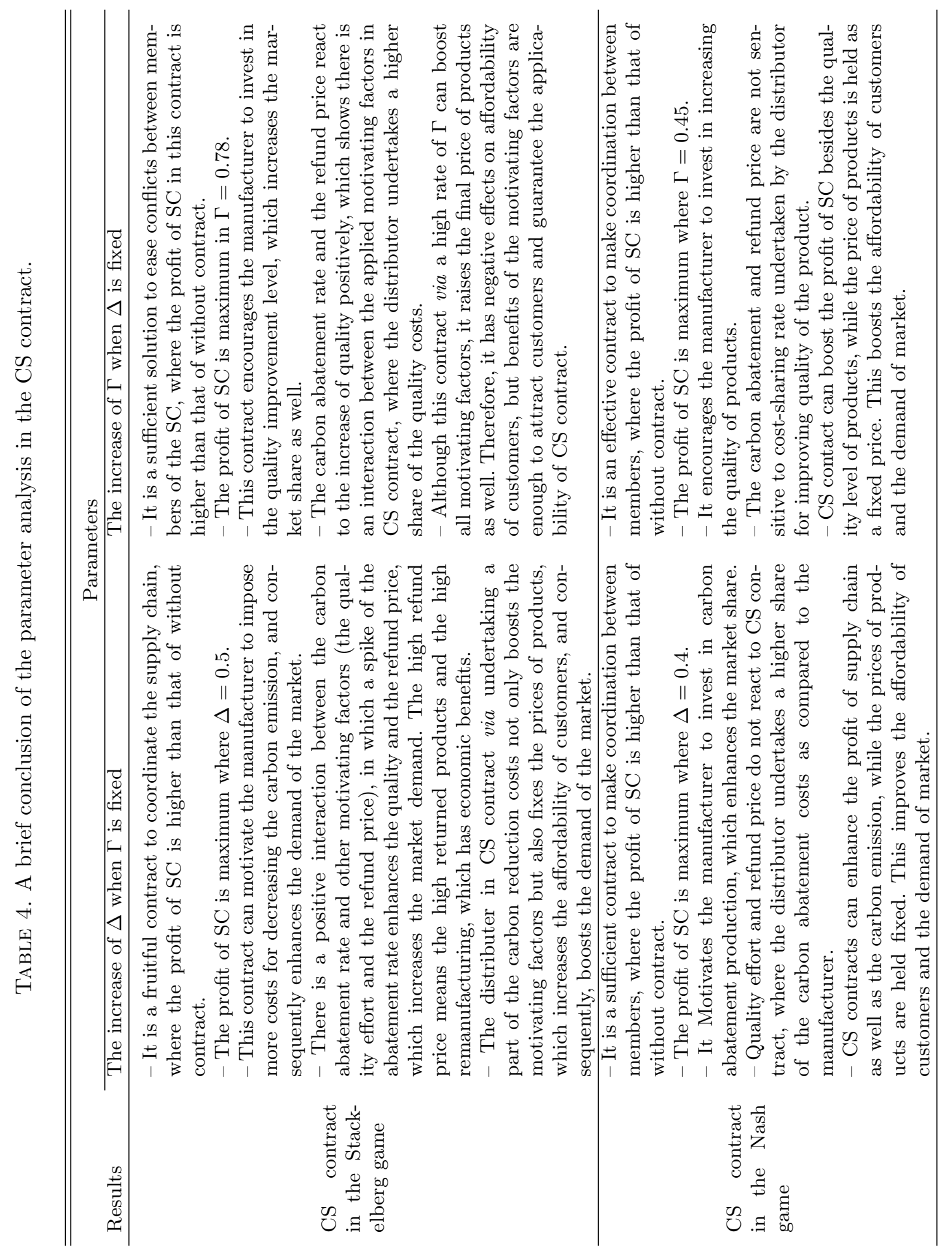


approaches including investment in new technologies to produce low-carbon production, using the trade and cap regulation to control the emission as well as recollecting and remanufacturing the defected products were involved in this research to ease the amount of carbon emission in order to have a less undesired environmental effect and higher profit. To this aim, a two-stage supply chain consisting of one distributor, one manufacturer, and one product was considered. The market product demand was stochastic and depended on the carbon reduction rate, the quality improvement effort, the final price of a product, and the refund price of the defected product. The high costs of applying quality improvement efforts, remanufacturing, carbon reduction and their effects on the price and profits of the supply chain and its members needed to make cooperation between the distributor and the manufacturer. Therefore, in this research, we used a cooperative supply chain alongside a cost-sharing contract as solutions to boost the performance of the chain. In addition, we used game theory approaches including Nash and Stackelberg game (with the distributor as a leader) to obtain the optimal values of decision variables and the profits. The results showed that employing both the cooperative supply chain and the CS contract are effective solutions to boost SC's profit. Moreover, analyses of the parameters in the CS contract determined that in the Nash game there is no interaction between the carbon abatement, refund price, and quality level, while in the Stackelberg game positive relations among the motivating factors appeared. More specifically, in the Stackelberg game, the distributor undertaking a part of costs could enhance all motivating factors and boost the market share of the SC.

Although we applied different motivating factors to attract customers in a supply chain, there are some other factors such as an advertisement, delivery time, online or direct selling approaches which can be added in future investigations. In addition, as we considered stochastic demand, other inventory control parameters like salvage value or lead time can be considered in further studies. Moreover, we just focused on a CS contract and a cooperative model to simplify the production flow between the members. However, there are some other contracts such as revenue sharing, two-part tariff, or quantity discount contracts that can be used in the future.

\section{Appendix A. Proof of Theorem 4.1. The Cooperative supply Chain}

In order to prove the concavity of the chain profit in the cooperative case (shown in Eq. (4.1)), the Hessian matrix is obtained as:

$$
H(s, r, q, p, I)=\left[\begin{array}{ccccc}
2 p_{s} \lambda \beta_{2}-\phi & p_{s} \lambda \beta_{3} & p_{s} \lambda \beta_{4} & \beta_{2}-\beta_{1} p_{s} \lambda & p_{s} \lambda \\
p_{s} \lambda \beta_{3} & -2 \delta & \gamma & \beta_{3} & 0 \\
p_{s} \lambda \beta_{4} & \gamma & -2 \theta & \beta_{4} & 0 \\
\beta_{2}-\beta_{1} p_{s} \lambda & \beta_{3} & \beta_{4} & -2 \beta_{1} & 1-\frac{I}{B} \\
p_{s} \lambda & 0 & 0 & 1-\frac{I}{B} & -\frac{p+h^{+}+h^{-}}{B}
\end{array}\right] .
$$

To prove the concavity, the following conditions must hold:

$$
\begin{aligned}
\left|H_{1}(s, r, q, p, I)\right| & =2 p_{s} \lambda \beta_{2}-\phi<0 \\
\left|H_{2}(s, r, q, p, I)\right| & =p_{s}^{2} \lambda^{2} \beta_{3}^{2}<-2 \delta\left(2 p_{s} \lambda \beta_{2}-\phi\right) \\
\left|H_{3}(s, r, q, p, I)\right| & =\left(2 p_{s} \lambda \beta_{2}-\phi\right)\left|\begin{array}{cc}
-2 \delta & \gamma \\
\gamma & -2 \theta
\end{array}\right|-p_{s} \lambda \beta_{3}\left|\begin{array}{cc}
p_{s} \lambda \beta_{3} & \gamma \\
p_{s} \lambda \beta_{4} & -2 \theta
\end{array}\right|+p_{s} \lambda \beta_{4}\left|\begin{array}{cc}
p_{s} \lambda \beta_{3} & -2 \delta \\
p_{s} \lambda \beta_{3} & \gamma
\end{array}\right|<0
\end{aligned}
$$

which, upon simplification is:

$$
\left|H_{3}(s, r, q, p, I)\right|=A_{3} A_{4}(2 \theta+2 \gamma)+A_{2}\left(4 \delta \theta-\gamma^{2}\right)<2 \delta A_{4}^{2} .
$$


Additionally, the following inequality should be true.

$$
\begin{aligned}
\left|H_{4}(s, r, q, p, I)\right|= & \left(2 p_{s} \lambda \beta_{2}-\phi\right)\left|\begin{array}{ccc}
-2 \delta & \gamma & \beta_{3} \\
\gamma & -2 \theta & \beta_{4} \\
\beta_{3} & \beta_{4} & -2 \beta_{1}
\end{array}\right|-p_{s} \lambda \beta_{3}\left|\begin{array}{ccc}
p_{s} \lambda \beta_{3} & \gamma & \beta_{3} \\
p_{s} \lambda \beta_{4} & -2 \theta & \beta_{4} \\
\beta_{2}-\beta_{1} p_{s} \lambda & \beta_{4} & -2 \beta_{1}
\end{array}\right| \\
& +p_{s} \lambda \beta_{4}\left|\begin{array}{ccc}
p_{s} \lambda \beta_{3} & -2 \delta & \beta_{3} \\
p_{s} \lambda \beta_{4} & \gamma & \beta_{4} \\
\beta_{2}-\beta_{1} p_{s} \lambda & \beta_{3} & -2 \beta_{1}
\end{array}\right|-\left(\beta_{2}-\beta_{1} p_{s} \lambda\right)\left|\begin{array}{ccc}
p_{s} \lambda \beta_{3} & -2 \delta & \gamma \\
p_{s} \lambda \beta_{4} & \gamma & -2 \theta \\
\beta_{2}-\beta_{1} p_{s} \lambda & \beta_{3} & \beta_{4}
\end{array}\right|>0
\end{aligned}
$$

which yields to:

$$
\begin{aligned}
\left|H_{4}(s, r, q, p, I)\right|= & -A_{4}^{2}\left(\beta_{3}^{2}+4 \beta_{1} \delta\right)-4 \beta_{1} A_{3}^{2} \theta-2 A_{2} \beta_{3}^{2} \theta-8 A_{2} \beta_{1} \delta \theta+A_{3}^{2} \beta_{4}^{2}+2 A_{2} \delta \beta_{4}^{2}+2 A_{2} \beta_{1} \gamma^{2} \\
& -A_{4}\left(-A_{1} \beta_{3} \beta_{4}+2 A_{3} \beta_{3} \beta_{4}+4 A_{1} \delta \beta_{4}+4 A_{3} \beta_{1} \gamma+A_{1} \beta_{3} \gamma\right)-2 A_{1} A_{3}\left(2 \beta_{3} \theta+\beta_{4} \gamma\right) \\
& -A_{1}^{2}\left(4 \delta+\gamma^{2}\right)>0
\end{aligned}
$$

Finally, we need to have:

$$
\begin{aligned}
& \left|H_{3}(g, p, I)\right|
\end{aligned}
$$

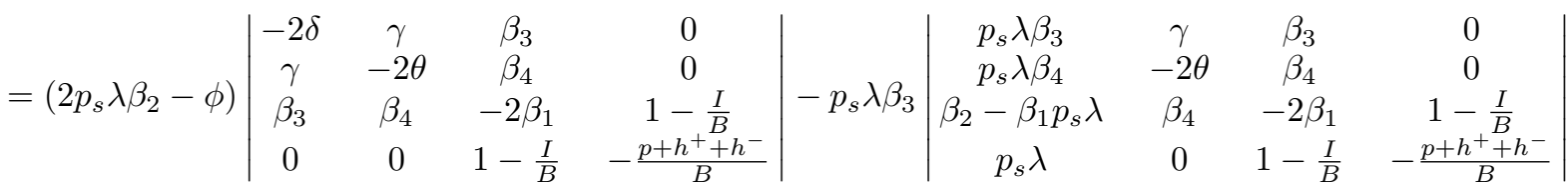

$$
\begin{aligned}
& +p_{s} \lambda \beta_{4}\left|\begin{array}{cccc}
p_{s} \lambda \beta_{3} & -2 \delta & \beta_{3} & 0 \\
p_{s} \lambda \beta_{4} & \gamma & \beta_{4} & 0 \\
\beta_{2}-\beta_{1} p_{s} \lambda & \beta_{3} & -2 \beta_{1} & 1-\frac{I}{B} \\
p_{s} \lambda & 0 & 1-\frac{I}{B} & -\frac{p+h^{+}+h^{-}}{B}
\end{array}\right| \\
& -\left(\beta_{2}-\beta_{1} p_{s} \lambda\right)\left|\begin{array}{cccc}
p_{s} \lambda \beta_{3} & -2 \delta & \gamma & 0 \\
p_{s} \lambda \beta_{4} & \gamma & -2 \theta & 0 \\
\beta_{2}-\beta_{1} p_{s} \lambda & \beta_{3} & \beta_{4} & 1-\frac{I}{B} \\
p_{s} \lambda & 0 & 0 & -\frac{p+h^{+}+h^{-}}{B}
\end{array}\right|<0
\end{aligned}
$$

which rearranged as:

$$
\begin{aligned}
& \alpha_{5}^{\mathrm{C}}=-\frac{1}{E}\left(2 E A_{5}^{2} E \beta_{3}^{2} \phi+4 A_{2} E^{2} \delta \phi-8 A_{1} A_{5} E^{2} \delta \phi-8 A_{5}^{2} E^{2} \beta_{1} \delta \phi+2 A_{2} E \beta_{3}^{2} h^{+} \phi-4 A_{1}^{2} E \delta h^{+} \phi\right. \\
& -8 A_{2} E \beta_{1} \delta h^{+} \phi+2 A_{5}^{2} E^{2} \delta \beta_{4}^{2}+2 A_{2} E \delta h^{+} \beta_{4}^{2}+2 A_{2} E \beta_{3}^{2} \phi p-4 A_{1}^{2} E \delta \phi p-8 A_{2} E \beta_{1} \delta \phi p+2 A_{2} E \delta \beta_{4}^{2} p \\
& +2 A_{2} E \beta_{3}^{2} \phi s-4 A_{1}^{2} E \delta \phi s-8 A_{2} E \beta_{1} \delta \phi h^{-}+2 A_{2} E \delta \beta_{4}^{2} h^{-}+A_{5}^{2} E^{2} \beta_{3} \beta_{4} \gamma+A_{5}^{2} E^{2} \beta_{3} \beta_{4} \gamma+2 A_{2} E \beta_{3} h^{+} \beta_{4} \gamma \\
& +2 A_{2} E \beta_{3} \beta_{4} p \gamma+2 A_{2} E \beta_{3} \beta_{4} h^{-} \gamma-A_{2} E^{2} \gamma^{2}+2 A_{1} A_{5} E^{2} \gamma^{2}+2 A_{5}^{2} E^{2} \beta_{1} \gamma^{2}+A_{1}^{2} E h^{+} \gamma^{2}+2 A_{2} E \beta_{1} h^{+} \gamma^{2} \\
& +A_{1}^{2} E p \gamma^{2}+2 A_{2} E \beta_{1} p \gamma^{2}+A_{1}^{2} E h^{-} \gamma^{2}+2 A_{2} E \beta_{1} h^{-} \gamma^{2}-2 A_{3} E\left(\beta_{3} \phi+\beta_{3} \phi+\beta_{4} \gamma\right)\left(A_{1}\left(h^{+}+p+h^{-}\right)\right. \\
& \left.+A_{5}(E-I)\right)-8 A_{2} E \delta \phi I+8 A_{1} A_{5} E \delta \phi I+2 A_{2} E \gamma^{2} I-2 A_{1} A_{5} E \gamma^{2} I+4 A_{2} \delta \phi I^{2} \\
& -A_{2} \gamma^{2} I^{2}+A_{3}^{2}\left(2 E^{2} \phi+2 \phi I^{2}+E\left(h^{+} \beta_{4}^{2}+\beta_{4}^{2} p+\beta_{4}^{2} h^{-}-4 \beta_{1} \phi\left(h^{+}+p+h^{-}\right)-4 \phi I\right)\right) \\
& +A_{4}^{2}\left(2 E^{2} \delta+2 \delta I^{2}+E\left(\beta_{3}^{2}\left(h^{+}+p+h^{-}\right)-4 \delta\left(\beta_{1}\left(h^{+}+p+h^{-}\right)+I\right)\right)\right) \\
& -A_{4}\left(\begin{array}{l}
A_{1} E\left(h^{+}+p+h^{-}\right)\left(4 \delta \beta_{4}+\left(2 \beta_{3}\right) \gamma\right)+A_{5} E\left(4 \delta \beta_{4}+2 \beta_{3} \gamma\right)(E-I) \\
+2 A_{3}\left(-E^{2} \gamma-\gamma I^{2}+E\left(\beta_{3} \beta_{4}\left(h^{+}+p+h^{-}\right)+2 \gamma\left(\beta_{1}\left(h^{+}+p+h^{-}\right)+I\right)\right)\right)
\end{array}\right)<0
\end{aligned}
$$

where constant parameters $A_{1}-A_{5}$ are $A_{1}=\beta_{2}-\left(\beta_{1} p_{s} \lambda\right), A_{2}=\left(2 p_{s} \lambda \beta_{2}\right)-\phi, A_{3}=p_{s} \lambda \beta_{3}, A_{4}=p_{s} \lambda \beta_{4}$, $A_{5}=p_{s} \lambda$. Afterward, the optimal values of the decision variables are obtained by taking the partial derivatives 
of the objective function shown in equation (4.1) with respect to the decision variables and setting all equal zero. In other words, we have:

$$
\begin{aligned}
& \frac{\partial \prod^{\mathrm{C}}}{\partial r}=\beta_{3} p+p_{s} \lambda \beta_{3} s-2 \delta r+\gamma q+T \delta-\left(\alpha+\beta_{3}\left(c_{m}+p_{s} \lambda\right)\right)=0 \\
& \frac{\partial \prod^{\mathrm{C}}}{\partial q}=\beta_{4} p+p_{s} \lambda \beta_{4} s-2 \theta q+\gamma r+T \gamma-\beta_{4}\left(c_{m}+p_{s} \lambda\right)=0 \\
& \frac{\partial \prod^{\mathrm{C}}}{\partial p}=-2 \beta_{1} p+\left(\beta_{3}-\beta_{1} p_{s} \lambda\right) s+\beta_{3} r+\beta_{4} q+I-\left(I^{2} / 2 E\right)+\beta_{0}+\beta_{1}\left(c_{m}+p_{s} \lambda\right)=0 \\
& \frac{\partial \prod^{\mathrm{C}}}{\partial I}=p+p_{s} \lambda s+h^{-}-c_{m}-p_{s} \lambda-\left(p+h^{+}+h^{-} / E\right) I=0 \\
& \frac{\partial \prod^{\mathrm{C}}}{\partial s}=\left(\beta_{2}-\beta_{1} p_{s} \lambda\right) p+\left(2 p_{s} \lambda \beta_{2}-\phi\right) s+p_{s} \lambda \beta_{3} r+p_{s} \lambda \beta_{4} q+p_{s} \lambda I+p_{s} \lambda \beta_{0}-\left(c_{m}+p_{s} \lambda\right) \beta_{2}=0 .
\end{aligned}
$$

Then, solving equations (A.10) to (A.14) results in equations (4.2) to (4.6) presented in Theorem 4.1 in which constant parameters $A_{6}-A_{26}$ and $H_{1}-H_{5}$ are used for a better presentation of the closed-form equations of the variables as

$$
\begin{aligned}
A_{6}= & p_{s} \lambda \beta_{0}-\left(C_{m}+p_{s} \lambda\right) \beta_{2}, \quad A_{7}=T \delta-\left(\alpha+\beta_{3}\left(C_{m}+p_{s} \lambda\right)\right), \quad A_{8}=T \gamma+\beta_{4}\left(C_{m}+p_{s} \lambda\right), \\
A_{9}= & \beta_{0}+\beta_{1}\left(C_{m}+p_{s} \lambda\right), \quad A_{10}=h^{-}-C_{m}-p_{s} \lambda, \quad A_{11}=2 \delta \beta_{4}+\beta_{3} \gamma, \quad A_{12}=2 \delta A_{4} A_{3} \gamma, \quad A_{13}=\gamma^{2}-4 \theta \delta, \\
A_{14}= & A_{7} \gamma-A_{8} \delta, \quad A_{15}=\beta_{4}+\frac{2 \theta A_{11}}{A_{13}}, \quad A_{16}=A_{4}+\frac{2 \theta A_{12}}{A_{13}}, \quad A_{17}=\frac{2 \theta A_{14}}{A_{13}}-A_{8}, \quad A_{18}=A_{1}-\frac{A_{3} A_{15}}{\gamma} \\
& -\frac{A_{4} A_{11}}{A_{13}}, \quad A_{19}=A_{2}-\frac{A_{3} A_{16}}{\gamma}-\frac{A_{4} A_{12}}{A_{13}}, \quad A_{20}=A_{6}-\frac{A_{4} A_{14}}{A_{13}}-\frac{A_{3} A_{17}}{\gamma}, \quad A_{21}=-2 \beta_{1}-\frac{A_{1} A_{18}}{A_{19}} \\
& -\frac{\beta_{3} A_{15}}{\gamma}+\frac{\beta_{3} A_{16} A_{18}}{A_{19} \gamma}-\frac{\beta_{4} A_{11}}{A_{13}}+\frac{\beta_{4} A_{12} A_{18}}{A_{13} A_{19}}, \quad A_{22}=-\frac{A_{1} A_{5}}{A_{19}}+\frac{\beta_{3} A_{5} A_{16}}{A_{19} \gamma}+\frac{\beta_{4} A_{12} A_{5}}{A_{13} A_{19}}+1, \\
A_{23}= & A_{9}-\frac{A_{1} A_{20}}{A_{19}}+\frac{\beta_{3} A_{16} A_{20}}{A_{19} \gamma}-\frac{\beta_{3} A_{18}}{\gamma}+\frac{\beta_{4} A_{12} A_{20}}{A_{13} A_{19}}-\frac{\beta_{4} A_{14}}{A_{13}}, \quad A_{24}=1-\frac{A_{5} A_{18}}{A_{19}}, \quad A_{25}=\frac{A_{5}^{2}}{A_{19}}, \\
A_{26}= & A_{10}-A_{5} A_{20}, \quad\left(2 A_{21} E h^{+}\right) / 3 \\
H_{1}= & \left(-\left(2 A_{23} E\right) / 3\right)+(2 / 3) A_{22} A_{24} E^{2}+(2 / 3) A_{21} A_{25} E^{2}-(1 / 9)\left(2 A_{22} E+A_{24} E\right)^{2}+\left(2 A_{21}\right. \\
& +\left(2 A_{21} E h^{-}\right) / 3, \quad H_{2}=-A_{23} A_{24} E^{2}+A_{21} A_{26} E^{2}+\frac{1}{27}\left(2 A_{22} E+A_{24} E\right)^{3}, \\
H_{3}= & \frac{1}{6}\left(2 A_{22} E+A_{24} E\right)\left(-2 A_{23} E+2 A_{22} A_{24} E^{2}+2 A_{21} A_{25} E^{2}+2 A_{21} E h^{+}+2 A_{21} E h^{-}\right), \\
H_{4}= & A_{23} A_{24} E^{2}-A_{21} A_{26} E^{2}-\frac{1}{27}\left(2 A_{22} E+A_{24} E\right)^{3}, \quad H_{5}=\sqrt{\left(H_{1}\right)^{3}+\left(H_{4}+H_{3}\right)^{2} .}
\end{aligned}
$$

\section{Appendix B. Proof of Theorem 4.2. The manufacturer's optimum Variables in THE NASH GAME}

The Hessian matrix of the manufacturer profit function in the Nash game is as follows:

$$
H(s, r, q, w)=\left[\begin{array}{cccc}
2 p_{s} \lambda \beta_{2}-\phi & p_{s} \beta_{3} \lambda & p_{s} \beta_{4} \lambda & \beta_{2}-\beta_{1} p_{s} \lambda \\
p_{s} \beta_{3} \lambda & -2 \delta & \gamma & \beta_{3} \\
p_{s} \beta_{4} \lambda & \gamma & -2 \theta & \beta_{4} \\
\beta_{2}-\beta_{1} p_{s} \lambda & \beta_{3} & \beta_{4} & -2 \beta_{1}
\end{array}\right]
$$


Then, the following conditions must hold in order to prove that the manufacturer profit function is concave:

$$
\begin{aligned}
\left|H_{1}(s, r, q, w)\right| & =2 p_{s} \lambda \beta_{2}-\phi<0 \\
\left|H_{2}(s, r, q, w)\right| & =2 \phi \delta-p_{s}^{2} \beta_{3}^{2} \lambda^{2}-4 p_{s} \lambda \delta \beta_{2}>0 \\
\left|H_{3}(s, r, q, w)\right| & =\left(2 p_{s} \lambda \beta_{2}-\phi\right)\left|\begin{array}{cc}
-2 \delta & \gamma \\
\gamma & -2 \theta
\end{array}\right|-p_{s} \beta_{3} \lambda\left|\begin{array}{cc}
p_{s} \beta_{3} \lambda & \gamma \\
p_{s} \beta_{4} \lambda & -2 \theta
\end{array}\right|+p_{s} \beta_{4} \lambda\left|\begin{array}{cc}
p_{s} \beta_{3} \lambda & -2 \delta \\
p_{s} \beta_{4} \lambda & \gamma
\end{array}\right|<0
\end{aligned}
$$

which results in:

$$
\left|H_{3}(s, r, q, w)\right|=2 B_{4} B_{5} \gamma+2 \theta B_{4}^{2}-2 \delta B_{5}^{2}+B_{7}\left(4 \delta \theta-\gamma^{2}\right)<0 .
$$

Finally,

$$
\begin{aligned}
\left|H_{4}(s, r, q, w)\right|= & \left(2 p_{s} \lambda \beta_{2}-\phi\right)\left|\begin{array}{ccc}
-2 \delta & \gamma & \beta_{3} \\
\gamma & -2 \theta & \beta_{4} \\
\beta_{3} & \beta_{4} & -2 \beta_{1}
\end{array}\right|-p_{s} \beta_{3} \lambda\left|\begin{array}{ccc}
p_{s} \beta_{3} \lambda & \gamma & \beta_{3} \\
p_{s} \beta_{4} \lambda & -2 \theta & \beta_{4} \\
\beta_{2}-\beta_{1} p_{s} \lambda & \beta_{4} & -2 \beta_{1}
\end{array}\right| \\
& +p_{s} \beta_{4} \lambda\left|\begin{array}{ccc}
p_{s} \beta_{3} \lambda & -2 \delta & \beta_{3} \\
p_{s} \beta_{4} \lambda & \gamma & \beta_{4} \\
\beta_{2}-\beta_{1} p_{s} \lambda & \beta_{3} & -2 \beta_{1}
\end{array}\right|-\left(\beta_{2}-\beta_{1} p_{s} \lambda\right)\left|\begin{array}{ccc}
p_{s} \beta_{3} \lambda & -2 \delta & \gamma \\
p_{s} \beta_{4} \lambda & \gamma & -2 \theta \\
\beta_{2}-\beta_{1} p_{s} \lambda & \beta_{3} & \beta_{4}
\end{array}\right|>0 .
\end{aligned}
$$

By simplification we have:

$$
\begin{aligned}
\left|H_{4}(s, r, q, w)\right|= & B_{5}^{2}\left(\beta_{3}^{2}-4 \beta_{1} \delta\right)+2 B_{7} \beta_{3}^{2} \theta-4 B_{1}^{2} \delta \theta-8 B_{7} \beta_{1} \delta \theta+2 B_{7} \delta \beta_{4}^{2}+B_{4}^{2}\left(-4 \beta_{1} \theta+\beta_{4}^{2}\right) \\
& +2 B_{7} \beta_{3} \beta_{4} \gamma+B_{1}^{2} \gamma^{2}+2 B_{7} \beta_{1} \gamma^{2}-2 B_{5}\left(B_{4} \beta_{3} \beta_{4}+2 B_{1} \delta \beta_{4}+2 B_{4} \beta_{1} \gamma+B_{1} \beta_{3} \gamma\right) \\
& -2 B_{1} B_{4}\left(2 \beta_{3} \theta+\beta_{4} \gamma\right)>0
\end{aligned}
$$

where the constants $B_{1}-B_{7}$ are defined as $B_{1}=\beta_{2}-\beta_{1} p_{S} \lambda, B_{2}=\beta_{0}+\beta_{1}\left(C_{m}+p_{s} \lambda\right), B_{3}=\beta_{1} p_{S} \lambda, B_{4}=\beta_{3} p_{S} \lambda$, $B_{5}=\beta_{4} p_{S} \lambda, B_{6}=p_{s} \lambda, B_{7}=2 \lambda p_{s} \beta_{2}-\phi$.

Hence, Taking the partial derivatives of the manufacturer's profit function (Eq. (3.5)) with respect to his decision variables and setting all equal zero yields to equations (B.8) to (B.11).

$$
\begin{aligned}
\frac{\partial \prod_{\mathrm{M}}}{\partial w}= & -2 \beta_{1} w-\beta_{1} m+\left(\beta_{2}-\beta_{1} p_{s} \lambda\right) s+\beta_{3} r+\beta_{4} q+I+\beta_{0}+\beta_{1}\left(C_{m}+p_{s} \lambda\right)=0 \\
\frac{\partial \prod_{\mathrm{M}}}{\partial s}= & \left(\beta_{2}-\beta_{1} p_{s} \lambda\right) w-\beta_{1} p_{s} \lambda m+\left(2 p_{s} \lambda \beta_{2}-\phi\right) s+p_{s} \beta_{3} \lambda r+p_{s} \beta_{4} \lambda q+p_{s} \lambda I+p_{s} \lambda \beta_{0} \\
& -\beta_{2}\left(c_{m}+p_{s} \lambda\right)=0 \\
\frac{\partial \prod_{\mathrm{M}}}{\partial r}= & \beta_{3} w+p_{s} \lambda \beta_{3} s+\gamma q-2 \delta r+T \delta-\alpha+\beta_{3}\left(C_{m}+p_{s} \lambda\right)=0 \\
\frac{\partial \prod_{\mathrm{M}}}{\partial q}= & \beta_{4} w+p_{s} \lambda \beta_{4} s+\gamma r-2 \theta q-\beta_{4}\left(C_{m}+p_{s} \lambda\right)=0 .
\end{aligned}
$$

Then solving equations (B.8) to (B.11) results in closed-form equations in Theorem 4.2 in which constant parameters $B_{8}-B_{36}$ are defined as

$$
\begin{aligned}
B_{8}= & p_{s} \lambda \beta_{0}-\beta_{2}\left(C_{m}+p_{s} \lambda\right), \quad B_{9}=T \delta-\alpha+\beta_{3}\left(C_{m}+p_{s} \lambda\right), \quad B_{10}=\beta_{4}\left(C_{m}+p_{s} \lambda\right)+T \gamma, \quad B_{11}=2 \theta \beta_{3}+\gamma \beta_{4}, \\
B_{12}= & 2 \theta B_{4}+B_{5} \gamma, \quad B_{13}=\gamma^{2}-4 \theta \delta, \quad B_{14}=B_{10} \gamma-2 \theta B_{9}, \quad B_{15}=\beta_{4}-\frac{B_{11} \gamma}{B_{13}}, \quad B_{16}=B_{5}-\frac{\gamma B_{12}}{B_{13}}, \\
B_{17}= & \frac{\gamma B_{14}}{B_{13}}-B_{10}, \quad B_{18}=\frac{\beta_{4} B_{15}}{2 \theta}-2 \beta_{1}-\frac{\beta_{4} B_{11}}{B_{13}}, \quad B_{19}=B_{1}-\frac{\beta_{3} B_{12}}{B_{13}}+\frac{\beta_{4} B_{16}}{2 \theta}, \quad B_{20}=B_{2}+\frac{\beta_{3} B_{14}}{B_{13}} \\
& +\frac{\beta_{4} B_{17}}{2 \theta}, \quad B_{21}=B_{1}-\frac{B_{4} B_{11}}{B_{13}}+\frac{B_{5} B_{15}}{2 \theta}, \quad B_{22}=B_{7}-\frac{B_{4} B_{12}}{B_{13}}+\frac{B_{5} B_{16}}{2 \theta}, \quad B_{23}=B_{8}-\frac{B_{4} B_{14}}{B_{13}}
\end{aligned}
$$




$$
\begin{aligned}
& +\frac{B_{5} B_{17}}{2 \theta}, \quad B_{24}=B_{21} B_{19}-B_{18} B_{22}, \quad B_{25}=B_{3} B_{18}-\beta_{1} B_{21}, \quad B_{26}=B_{21}-B_{6} B_{18}, \\
B_{27}= & B_{20} B_{21}-B_{18} B_{23}, \quad B_{28}=\frac{B_{22} B_{25}}{B_{24}}+B_{3}, \quad B_{29}=B_{6}-\frac{B_{22} B_{26}}{B_{24}}, \quad B_{30}=B_{23}-\frac{B_{22} B_{27}}{B_{24}}, \\
B_{31}= & -2 \beta_{1}-\frac{\beta_{1} B_{28}}{B_{21}}-\frac{\beta_{2} B_{25}}{B_{24}}-\frac{\beta_{3} B_{11} B_{28}}{B_{13} B_{21}}+\frac{\beta_{3} B_{12} B_{25}}{B_{13} B_{24}}+\frac{\beta_{4} B_{15} B_{28}}{2 \theta B_{21}}, \\
B_{32}= & 1+\frac{\beta_{1} B_{29}}{B_{21}}-\frac{\beta_{2} B_{26}}{B_{24}}+\frac{\beta_{3} B_{11} B_{29}}{B_{13} B_{21}}+\frac{\beta_{3} B_{12} B_{26}}{B_{13} B_{24}}-\frac{\beta_{4} B_{15} B_{29}}{2 \theta B_{21}}, \\
B_{33}= & \beta_{0}+\frac{\beta_{1} B_{30}}{B_{21}}-\frac{\beta_{2} B_{27}}{B_{24}}+\frac{\beta_{3} B_{11} B_{30}}{B_{13} B_{21}}+\frac{\beta_{3} B_{12} B_{27}}{B_{13} B_{24}}+\frac{\beta_{3} B_{14}}{B_{13}}-\frac{\beta_{4} B_{15} B_{30}}{2 B_{21} \theta}, \quad B_{34}=\frac{1+\frac{B_{28}}{B_{21}}}{E} \\
B_{35}= & \frac{B_{29}}{B_{21} E}, \quad B_{36}=\frac{h^{-}+h^{+}-\frac{B_{30}}{B_{21}}}{E} .
\end{aligned}
$$

\section{Appendix C. Proof of Theorem 4.3. Distributor's optimum variables in the NASH GAME}

In this case, the Hessian matrix of the distributor's profit function (Eq. (3.12)) is:

$$
H(m, I)=\left[\begin{array}{cc}
-2 \beta_{1} & 1-\frac{I}{E} \\
1-\frac{I}{E} & -\frac{h^{+}+h^{-}+m+w}{E}
\end{array}\right] .
$$

Then, the following conditions must hold:

$$
\begin{aligned}
& \left|H_{1}(m, I)\right|=-2 \beta_{1}<0 \\
& \left|H_{2}(m, I)\right|=\left(-2 \beta_{1}\right)\left(-\frac{h^{+}+h^{-}+m+w}{E}\right)-\left(1-\frac{I}{E}\right)^{2}>0 .
\end{aligned}
$$

The above inequality to be true require the following equation where optimal equation (4.11) is replaced instead of $w$.

$$
\left|H_{2}(m, I)\right|=-B_{31} B_{34} m+\left(2 B_{31} B_{35}+\frac{1}{E}+B_{32} B_{34}\right) I-\left(B_{32}+B_{31} B_{36}\right)-B_{34} \frac{I^{2}}{2 E}>0 .
$$

Then, the optimal values of the distributor's decision variables in the Nash game are obtained by taking the partial derivatives of his/her profit function (Eq. (3.12)) with respect to his/her decision variables, setting both equal to zero. Therefore:

$$
\begin{aligned}
\frac{\partial \prod_{\mathrm{R}}}{\partial m} & =\beta_{0}-\beta_{1} w-2 \beta_{1} m+\beta_{2} s+\beta_{3} r+\beta_{\partial q}+I-\frac{I^{2}}{2 E} \\
\frac{\partial \prod_{\mathrm{R}}^{\mathrm{CS}-N}}{\partial I} & =m+h^{-}-\left(m+w+h^{+}+h^{-}\right) I / E=0 .
\end{aligned}
$$

In order to solve equations (C.5) and (C.6), the optimum variables of manufacturer achieved from Theorem 4.2 are replaced into the mentioned equations. The substitutions rearrange the equations (C.5) and (C.6) as:

$$
\begin{aligned}
\frac{\partial \prod_{\mathrm{R}}}{\partial m}= & \left(\frac{\beta_{3} B_{12} B_{25}}{B_{13} B_{24}}-\frac{\beta_{1} B_{28}}{B_{21}}-\frac{\beta_{3} B_{25}}{B_{24}}-\frac{\beta_{3} B_{11} B_{28}}{B_{13} B_{21}}+\frac{\beta_{4} B_{15} B_{28}}{2 \theta B_{21}}-2 \beta_{1}\right) m+\frac{\beta_{3} B_{14}}{B_{13}}-\frac{\beta_{4} B_{15} B_{30}}{2 \theta B_{21}} \\
& +\left(\frac{\beta_{1} B_{29}}{B_{21}}-\frac{\beta_{3} B_{26}}{B_{24}}+\frac{\beta_{3} B_{12} B_{29}}{B_{13} B_{21}}+\frac{\beta_{3} B_{12} B_{26}}{B_{13} B_{24}}-\frac{\beta_{4} B_{15} B_{29}}{2 \theta B_{21}}+1\right) I-\frac{I^{2}}{2 E}+\beta_{0}+\frac{\beta_{1} B_{30}}{B_{21}}-\frac{\beta_{2} B_{27}}{B_{24}}
\end{aligned}
$$




$$
\begin{gathered}
+\frac{\beta_{3} B_{11} B_{30}}{B_{13} B_{21}}+\frac{\beta_{3} B_{12} B_{27}}{B_{13} B_{24}}=0 \\
\frac{\partial \prod_{\mathrm{R}}}{\partial I}=m+h^{-}-\left(\frac{1+\left(\frac{B_{28}}{B_{21}}\right)}{E}\right) m I+\frac{B_{29}}{B_{21} E} I^{2}-\left(\frac{\left(h^{+}+h^{-}-\frac{B_{30}}{B_{21}}\right)}{E}\right) I=0 .
\end{gathered}
$$

Solving nonlinear equations (C.7) and (C.8) leads into closed-form equations for the optimal values of the distributor's decision variables in equations (4.12) and (4.13) in Theorem 4.3 in which constant parameters $F_{1}$ to $F_{7}$ used in Theorem 4.3 are

$$
\begin{aligned}
F_{1}= & \frac{\left(1+2 E B_{32} B_{34}+2 E B_{31} B_{35}\right)^{2}}{\left(9 B_{34}^{2}\right)}, \quad F_{2}=\frac{2 E B_{32}-2 E B_{33} B_{34}+2 E B_{31} B_{36}}{3 B_{34}}, \\
F_{3}= & \frac{\left(1+2 E B_{32} B_{34}+2 E B_{31} B_{35}\right)^{3}}{27 B_{34}^{3}}, \quad F_{4}=\frac{1}{6 B_{34}^{2}}\left(1+2 E B_{32} B_{34}+2 E B_{31} B_{35}\right) \\
& \times\left(2 E B_{32}-2 E B_{33} B_{35}+2 E B_{31} B_{36}\right), \\
F_{5}= & \left(2 E B_{33}-2 E B_{31} h^{-}\right) /\left(2 B_{34}\right), \quad F_{6}=\sqrt{-\left(F_{1}-F_{2}\right)^{3}+\left(-F_{3}+F_{4}+F_{5}\right)^{2}}, \\
F_{7}= & \left(F_{3}-F_{4}-F_{5}+F_{6}\right)^{1 / 3} .
\end{aligned}
$$

\section{Appendix D. Proof of Theorem 4.5. Distributor's optimum values in the StaCkelBerg Game}

The Hessian matrix of the distributor's profit function (4.18) in the Stackelberg game is:

$$
H(m, I)=\left[\begin{array}{cc}
\frac{\partial^{2} \prod_{\mathrm{R}}^{\mathrm{SR}}}{\partial m^{2}} & \frac{\partial^{2} \prod_{\mathrm{R}}^{\mathrm{SR}}}{\partial I \partial m} \\
\frac{\partial^{2} \prod_{\mathrm{R}}^{\mathrm{SR}}}{\partial m \partial I} & \frac{\partial^{2} \prod_{\mathrm{R}}^{\mathrm{SR}}}{\partial I^{2}}
\end{array}\right]=\left[\begin{array}{cc}
-2 S_{1} & S_{2}-\left(\frac{\left(1+B_{28} / B_{21}\right)}{E}\right) I \\
S_{2}-\left(\frac{\left(1+B_{28} / B_{21}\right)}{E}\right) I & -\left(\frac{\left(1+B_{28} / B_{21}\right)}{E}\right) m+\frac{3 S_{5}}{E} I
\end{array}\right] .
$$

Then, the following conditions must hold to ensure the concavity of the distributor's profit function:

$$
\begin{aligned}
& \left|H_{1}(m, I)\right|=\frac{\partial^{2} \prod_{\mathrm{R}}^{\mathrm{SR}}}{\partial m^{2}}=-2 S_{1}<0 \\
& \left|H_{2}(m, I)\right|=\left(4 S_{1} S_{10}+2 S_{2} S_{8}\right) I-S_{8}^{2} I^{2}-2 S_{1} S_{8} m-S_{2}^{2}<0
\end{aligned}
$$

where constant parameters $S_{1}$ to $S_{10}$ are defined as

$$
\begin{aligned}
& S_{1}=-\beta_{1}-\frac{\beta_{1} B_{28}}{B_{21}}-\frac{\beta_{2} B_{25}}{B_{24}}-\frac{\beta_{3} B_{11} B_{28}}{B_{13} B_{21}}+\frac{\beta_{3} B_{12} B_{25}}{B_{13} B_{24}}-\frac{\beta_{4} B_{15} B_{28}}{2 \theta B_{21}}-\frac{\beta_{4} B_{16} B_{25}}{2 B_{24} \theta}, \\
& S_{2}=\frac{\beta_{1} B_{29}}{B_{21}}-\frac{\beta_{2} B_{26}}{B_{24}}+\frac{\beta_{3} B_{11} B_{29}}{B_{13} B_{21}}+\frac{\beta_{3} B_{12} B_{26}}{B_{13} B_{24}}-\frac{\beta_{4} B_{15} B_{29}}{2 \theta B_{21}}-\frac{\beta_{4} B_{16} B_{26}}{2 \theta B_{24}}+1, \\
& S_{3}=\beta_{0}+\frac{\beta_{1} B_{30}}{B_{21}}-\frac{\beta_{2} B_{27}}{B_{24}}+\frac{\beta_{3} B_{11} B_{30}}{B_{13} B_{21}}+\frac{\beta_{3} B_{12} B_{27}}{B_{13} B_{24}}+\frac{\beta_{3} B_{14}}{B_{13}}-\frac{\beta_{4} B_{15} B_{30}}{2 \theta B_{21}}-\frac{\beta_{4} B_{16} B_{27}}{2 \theta B_{24}}+\frac{\beta_{4} B_{17}}{2 \theta}, \\
& S_{4}=1+\frac{B_{28}}{B_{21}}, \quad S_{5}=\frac{B_{29}}{B_{21}}, \quad S_{6}=h^{+}+h^{-}-\frac{B_{30}}{B_{21}}, \quad S_{7}=\frac{S_{4}}{2 E}, \quad S_{8}=\frac{S_{4}}{E}, \quad S_{9}=\frac{S_{6}}{E}, \quad S_{10}=\frac{3 S_{5}}{2 E} .
\end{aligned}
$$

In order to find optimal $m^{\mathrm{SR}}$ and $I^{\mathrm{SR}}$, taking the partial derivative of the distributor's profit function (4.18) with respect to the distributor's decision variables, and setting both equal to zero yields

$$
\begin{aligned}
& \frac{\partial \prod_{\mathrm{R}}}{\partial m}=S_{2} I+S_{3}+2 S_{1} m-S_{4}\left(I^{2} / 2 E\right)=0 \\
& \frac{\partial \prod_{\mathrm{R}}}{\partial I}=\frac{3 S_{5}}{2 E} I^{2}+S_{2} m-\frac{S_{4}}{E} m I-\frac{S_{6}}{E} I+h^{-}=0 .
\end{aligned}
$$


Then, solving the nonlinear equations (D.5) and (D.6) leads to the results shown in Theorem 4.5. Moreover, other constant parameters $S_{11}-S_{16}$ to show the simple closed-form equations to obtain $m^{\mathrm{SR}}$ and $I^{\mathrm{SR}}$ are

$$
\begin{aligned}
& S_{11}=\frac{\left(2 S_{1} S_{10}+S_{2} S_{7}+S_{2} S_{8}\right)^{2}}{\left(9 S_{7}^{2} S_{8}^{2}\right)}-\frac{\left(S_{2}^{2}-S_{3} S_{8}+2 S_{1} S_{9}\right)}{\left(3 S_{7} S_{8}\right)}, \quad S_{12}=\frac{-2 h^{-} S_{1}+S_{2} S_{3}}{2 S_{7} S_{8}}, \\
& S_{13}=\frac{\left(2 S_{1} S_{10}+S_{2} S_{7}+S_{2} S_{8}\right)^{3}}{27 S_{7}^{3} S_{8}^{3}}, \quad S_{14}=\frac{\left(2 S_{1} S_{10}+S_{2} S_{7}+S_{2} S_{8}\right)\left(S_{2}^{2}-S_{3} S_{8}+2 S_{1} S_{9}\right)}{6 S_{7}^{2} S_{8}^{2}}, \\
& S_{15}=\sqrt{-S_{11}^{3}+\left(S_{12}-S_{13}+S_{14}\right)^{2}}, \quad S_{16}=\left(-S_{12}+S_{13}-S_{14}+S_{15}\right)^{1 / 3} .
\end{aligned}
$$

\section{Appendix E. Proof of Theorem 4.6. Manufacturer's optimum Decisions in CS-N}

The Hessian matrix of the manufacturer's profit function (Eq. (4.21)) is:

$$
H(s, r, q, w)=\left[\begin{array}{cccc}
2 p_{s} \lambda \beta_{2}-(1-\Delta) \phi & p_{s} \beta_{3} \lambda & p_{s} \beta_{4} \lambda & \beta_{2}-\beta_{1} p_{s} \lambda \\
p_{s} \beta_{3} \lambda & -2 \delta & \gamma & \beta_{3} \\
p_{s} \beta_{4} \lambda & \gamma & -2(1-\Gamma) \theta & \beta_{4} \\
\beta_{2}-\beta_{1} p_{s} \lambda & \beta_{3} & \beta_{4} & -2 \beta_{1}
\end{array}\right]
$$

Then, the following inequalities must hold:

$$
\begin{aligned}
& \left|H_{1}(s, r, q, w)\right|=2 p_{s} \lambda \beta_{2}-(1-\Delta) \phi<0 \\
& \left|H_{2}(s, r, q, w)\right|=2(1-\Delta) \phi \delta-p_{s}^{2} \beta_{3}^{2} \lambda^{2}-4 p_{s} \lambda \delta \beta_{2}>0 .
\end{aligned}
$$

In addition,

$$
\begin{aligned}
\left|H_{3}(s, r, q, w)\right|= & \left(2 p_{s} \lambda \beta_{2}-(1-\Delta) \phi\right)\left|\begin{array}{cc}
-2 \delta & \gamma \\
\gamma & -2(1-\Gamma) \theta
\end{array}\right|-p_{s} \beta_{3} \lambda\left|\begin{array}{cc}
p_{s} \beta_{3} \lambda & \gamma \\
p_{s} \beta_{4} \lambda & -2(1-\Gamma) \theta
\end{array}\right| \\
& +p_{s} \beta_{4} \lambda\left|\begin{array}{cc}
p_{s} \beta_{3} \lambda & -2 \delta \\
p_{s} \beta_{4} \lambda & \gamma
\end{array}\right|<0
\end{aligned}
$$

which upon simplification becomes:

$$
\left|H_{3}(s, r, q, w)\right|=2 B_{4}^{\prime} B_{5}^{\prime} \gamma+2(1-\Gamma) \theta B_{4}^{\prime 2}-2 \delta B_{5}^{\prime 2}+B_{7}^{\prime}\left(4 \delta(1-\Gamma) \theta-\gamma^{2}\right)<0 .
$$

Finally

$$
\begin{aligned}
\left|H_{4}(s, r, q, w)\right|= & \left(2 p_{s} \lambda \beta_{2}(1-\Delta) \phi\right)\left|\begin{array}{ccc}
-2 \delta & \gamma & \beta_{3} \\
\gamma & -2(1-\Gamma) \theta & \beta_{4} \\
\beta_{3} & \beta_{4} & -2 \beta_{1}
\end{array}\right| \\
& -p_{s} \beta_{3} \lambda\left|\begin{array}{ccc}
p_{s} \beta_{3} \lambda & \gamma & \beta_{3} \\
p_{s} \beta_{4} \lambda & -2(1-\Gamma) \theta & \beta_{4} \\
\beta_{2}-\beta_{1} p_{s} \lambda & \beta_{4} & -2 \beta_{1}
\end{array}\right| \\
& +p_{s} \beta_{4} \lambda\left|\begin{array}{ccc}
p_{s} \beta_{3} \lambda & -2 \delta & \beta_{3} \\
p_{s} \beta_{4} \lambda & \gamma & \beta_{4} \\
\beta_{2}-\beta_{1} p_{s} \lambda & \beta_{3} & -2 \beta_{1}
\end{array}\right|-\left(\beta_{2}-\beta_{1} p_{s} \lambda\right)\left|\begin{array}{ccc}
p_{s} \beta_{3} \lambda & -2 \delta & p_{s} \beta_{4} \lambda \\
\beta_{2}-\beta_{1} p_{s} \lambda & \gamma & -2(1-\Gamma) \theta \\
\beta_{3} & \beta_{4}
\end{array}\right|>0
\end{aligned}
$$


which leads to:

$$
\begin{aligned}
\left|H_{4}(s, r, q, w)\right|= & B_{5}^{\prime 2}\left(\beta_{3}^{2}-4 \beta_{1} \delta\right)+\left(2 B_{7}^{\prime} \beta_{3}^{2} \theta(1-\Gamma)-\left(4 B_{1}^{\prime 2} \delta(1-\Gamma) \theta-8 B_{7}^{\prime} \beta_{1} \delta(1-\Gamma) \theta+2 B_{7}^{\prime} \delta \beta_{4}^{2}\right.\right. \\
& +B_{4}^{\prime 2}\left(-4 \beta_{1} \theta(1-\Gamma)+\beta_{4}^{2}\right)+2 B_{7}^{\prime} \beta_{3} \beta_{4} \gamma+B_{1}^{2} \gamma^{2}+2 B_{7}^{\prime} \beta_{1} \gamma^{2} \\
& -2 B_{5}\left(B_{4} \beta_{3} \beta_{4}+2 B_{1} \delta \beta_{4}+2 B_{4} \beta_{1} \gamma+B_{1} \beta_{3} \gamma\right)-2 B_{1} B_{4}\left(2 \beta_{3} \theta(1-\Gamma)+\beta_{4}\right.
\end{aligned}
$$

where the constants $B_{1}^{\prime}-B_{7}^{\prime}$ are $B_{1}^{\prime}=\beta_{2}-\beta_{1} p_{S} \lambda, B_{2}^{\prime}=\beta_{0}+\beta_{1}\left(C_{m}+p_{s} \lambda\right), B_{3}^{\prime}=\beta_{1} p_{S} \lambda, B_{4}^{\prime}=\beta_{3} p_{S} \lambda$, $B_{5}^{\prime}=\beta_{4} p_{S} \lambda, B_{6}^{\prime}=p_{s} \lambda, B_{7}^{\prime}=2 \lambda p_{s}-(1-\Delta) \phi$.

In order to achieve the manufacturer's optimal solutions, Taking the partial derivatives of the manufacturer's profit function in the CS contract case (Eq. (4.21)) with respect to his/her decision variables and setting all equal zero results in equations (E.8) to (E.11). it may be noted that equation (4.7) is replaced instead of the final price of the product $(p)$ in equation (4.21).

$$
\begin{aligned}
& \frac{\partial \prod_{\mathrm{M}}^{\mathrm{CS}}}{\partial s}=\left(\beta_{2}-\beta_{1} p_{s} \lambda\right) w+\left(2 p_{s} \lambda \beta_{2}-(1-\Delta) \phi\right) s+\left(\beta_{3} r+\beta_{4} q+I+\beta_{0}-\beta_{1} m-\beta_{2}\right) p_{s} \lambda-\beta_{2} c_{m}=0 \\
& \frac{\partial \prod_{\mathrm{M}}^{\mathrm{CS}}}{\partial q}=\beta_{4} w+p_{s} \lambda \beta_{4} s+\gamma r-2(1-\Gamma) \theta q-\beta_{4}\left(c_{m}+p_{s} \lambda\right)=0 \\
& \frac{\partial \prod_{\mathrm{M}}}{\partial w}=-2 \beta_{1} w-\beta_{1} m+\left(\beta_{2}-\beta_{1} p_{s} \lambda\right) s+\beta_{3} r+\beta_{4} q+I+\beta_{0}+\beta_{1}\left(c_{m}+p_{s} \lambda\right)=0 \\
& \frac{\partial \prod_{\mathrm{M}}}{\partial r}=\beta_{3} w+p_{s} \lambda \beta_{3} s+\gamma q-2 \delta r+T \delta-\alpha+\beta_{3}\left(c_{m}+p_{s} \lambda\right)=0 .
\end{aligned}
$$

The proof of Theorem 4.6 is complete when equations (E.8) to (E.11) are solved simultaneously in which the constant parameters $B_{8}^{\prime}-B_{30}^{\prime}$ are

$$
\begin{aligned}
B_{8}^{\prime} & =p_{s} \lambda \beta_{0}-\beta_{2}\left(C_{m}+p_{s} \lambda\right), \quad B_{9}^{\prime}=T \delta-\alpha+\beta_{3}\left(C_{m}+p_{s} \lambda\right), \quad B_{10}^{\prime}=\beta_{4}\left(C_{m}+p_{s} \lambda\right)+T \gamma, \\
B_{11}^{\prime} & =2 \theta(1-\Gamma) \beta_{3}+\gamma \beta_{4}, \quad B_{12}^{\prime}=2 \theta(1-\Gamma) B_{4}^{\prime}+B_{5}^{\prime} \gamma, \quad B_{13}^{\prime}=\gamma^{2}-4 \theta(1-\Gamma) \delta, \\
B_{14}^{\prime} & =B_{10}^{\prime} \gamma-2 \theta(1-\Gamma) B_{9}^{\prime}, \quad B_{15}^{\prime}=\beta_{4}-\frac{B_{11}^{\prime} \gamma}{B_{13}^{\prime}}, \quad B_{16}^{\prime}=B_{5}^{\prime}-\frac{\gamma B_{12}^{\prime}}{B_{13}^{\prime}}, \quad B_{17}^{\prime}=\frac{\gamma B_{14}^{\prime}}{B_{13}^{\prime}-B_{10}^{\prime},} \\
B_{18}^{\prime} & =\frac{\beta_{4} B_{15}^{\prime}}{2 \theta(1-\Gamma)}-2 \beta_{1}-\frac{\beta_{4} B_{11}^{\prime}}{B_{13}^{\prime}}, \quad B_{19}^{\prime}=B_{1}^{\prime}-\frac{\beta_{3} B_{12}^{\prime}}{B_{13}^{\prime}}+\frac{\beta_{4} B_{16}^{\prime}}{2 \theta(1-\Gamma)}, \quad B_{20}^{\prime}=B_{2}^{\prime}+\frac{\beta_{3} B_{14}^{\prime}}{B_{13}^{\prime}}+\frac{\beta_{4} B_{17}^{\prime}}{2(1-\Gamma) \theta}, \\
B_{21}^{\prime} & =B_{1}^{\prime}-\frac{B_{4}^{\prime} B_{11}^{\prime}}{B_{13}^{\prime}}+\frac{B_{5}^{\prime} B_{15}^{\prime}}{2 \theta(1-\Gamma)}, \quad B_{22}^{\prime}=B_{7}^{\prime}-\frac{B_{4}^{\prime} B_{12}^{\prime}}{B_{13}^{\prime}}+\frac{B_{5}^{\prime} B_{16}^{\prime}}{2 \theta(1-\Gamma)}, \quad B_{23}^{\prime}=B_{8}^{\prime}-\frac{B_{4}^{\prime} B_{14}^{\prime}}{B_{13}^{\prime}}+\frac{B_{5}^{\prime} B_{17}^{\prime}}{2(1-\Gamma) \theta}, \\
B_{24}^{\prime} & =B_{21}^{\prime} B_{19}^{\prime}-B_{18}^{\prime} B_{22}^{\prime}, \quad B_{25}^{\prime}=B_{3}^{\prime} B_{18}^{\prime}-\beta_{1} B_{21}^{\prime}, \quad B_{26}^{\prime}=B_{21}^{\prime}-B_{6}^{\prime} B_{18}^{\prime}, \quad B_{27}^{\prime}=B_{20}^{\prime} B_{21}^{\prime}-B_{18}^{\prime} B_{23}^{\prime}, \\
B_{28}^{\prime} & =\frac{B_{22}^{\prime} B_{25}^{\prime}}{B_{24}^{\prime}}+B_{3}^{\prime}, \quad B_{29}^{\prime}=B_{6}^{\prime}-\frac{B_{22}^{\prime} B_{26}^{\prime}}{B_{24}^{\prime}}, \quad B_{30}^{\prime}=B_{23}^{\prime}-\frac{B_{22}^{\prime} B_{27}^{\prime}}{B_{24}^{\prime}} .
\end{aligned}
$$

\section{Appendix F. Proof of Theorem 4.7. The Distributor's optimization in CS-N}

In order to find the optimum distributor's variables, firstly, the profit function of the distributor in the CS-N case (shown in Eq. (4.22)) should be met, and to do so, the Hessian matrix of the distributor's profit function follows:

$$
H(m, I)=\left[\begin{array}{cc}
-2 \beta_{1} & 1-\frac{I}{E} \\
1-\frac{I}{E} & -\frac{h^{+}+h^{-}+m+w}{E}
\end{array}\right] .
$$

Then, the following conditions must hold:

$$
\begin{aligned}
& \left|H_{1}(m, I)\right|=-2 \beta_{1}<0 \\
& \left|H_{2}(m, I)\right|=\left(-2 \beta_{1}\right)\left(-\frac{h^{+}+h^{-}+m+w}{E}\right)-\left(1-\frac{I}{E}\right)^{2}>0
\end{aligned}
$$


which is simplified to the following form using optimal (4.26) for $w$.

$$
\left|H_{2}(m, I)\right|=-B_{31}^{\prime} B_{34}^{\prime} m+\left(2 B_{31}^{\prime} B_{35}^{\prime}+\frac{1}{E}+B_{32}^{\prime} B_{34}^{\prime}\right) I-\left(B_{32}^{\prime}+B_{31}^{\prime} B_{36}^{\prime}\right)-B_{34}^{\prime} \frac{I^{2}}{2 E}>0 .
$$

In order to find optimal variables of the distributor in CS contract under Nash game, setting $\partial \prod_{\mathrm{R}}^{\mathrm{CS}-\mathrm{N}} / \partial m$ and $\partial \prod_{\mathrm{R}}^{\mathrm{CS}-\mathrm{N}} / \partial I$ to zero the following equations are achieved:

$$
\begin{aligned}
& \frac{\partial \prod_{\mathrm{R}}^{\mathrm{CS}-\mathrm{N}}}{\partial m}=\beta_{0}-\beta_{1} w^{\mathrm{CS}-\mathrm{N}}-2 \beta_{1} m+\beta_{2} s^{\mathrm{CS}-\mathrm{N}}+\beta_{3} r^{\mathrm{CS}-\mathrm{N}}+\beta_{4} q^{\mathrm{CS}-\mathrm{N}}+I-\frac{I^{2}}{2 E}=0 \\
& \frac{\partial \prod_{\mathrm{R}}^{\mathrm{CS}-\mathrm{N}}}{\partial I}=m+h^{-}-\left(m+w^{\mathrm{CS}-\mathrm{N}}+h^{+}+h^{-}\right) I / E=0 .
\end{aligned}
$$

The solution of equations (F.5) and (F.6) yields to results in Theorem 4.7 in which constant parameters $B_{31}^{\prime}-B_{36}^{\prime}$ and $F_{1}^{\prime}-F_{7}^{\prime}$ are:

$$
\begin{aligned}
B_{31}^{\prime} & =-2 \beta_{1}-\frac{\beta_{1} B_{28}^{\prime}}{B_{21}^{\prime}}-\frac{\beta_{2} B_{25}^{\prime}}{B_{24}^{\prime}}-\frac{\beta_{3} B_{11}^{\prime} B_{28}^{\prime}}{B_{13}^{\prime} B_{21}^{\prime}}+\frac{\beta_{3} B_{12}^{\prime} B_{25}^{\prime}}{B_{13}^{\prime} B_{24}^{\prime}}+\frac{\beta_{4} B_{15}^{\prime} B_{28}^{\prime}}{2 \theta(1-\Gamma) B_{21}^{\prime}}, \\
B_{32}^{\prime} & =1+\frac{\beta_{1} B_{29}^{\prime}}{B_{21}^{\prime}}-\frac{\beta_{2} B_{26}^{\prime}}{B_{24}^{\prime}}+\frac{\beta_{3} B_{11}^{\prime} B_{29}^{\prime}}{B_{13}^{\prime} B_{21}^{\prime}}+\frac{\beta_{3} B_{12}^{\prime} B_{26}^{\prime}}{B_{13}^{\prime} B_{24}^{\prime}}-\frac{\beta_{4} B_{15}^{\prime} B_{29}^{\prime}}{2 \theta(1-\Gamma) B_{21}^{\prime}}, \\
B_{33}^{\prime} & =\beta_{0}+\frac{\beta_{1} B_{30}^{\prime}}{B_{21}^{\prime}}-\frac{\beta_{2} B_{27}^{\prime}}{B_{24}^{\prime}}+\frac{\beta_{3} B_{11}^{\prime} B_{30}^{\prime}}{B_{13}^{\prime} B_{21}^{\prime}}+\frac{\beta_{3} B_{12}^{\prime} B_{27}^{\prime}}{B_{13}^{\prime} B_{24}^{\prime}}+\frac{\beta_{3} B_{14}^{\prime}}{B_{13}^{\prime}}-\frac{\beta_{4} B_{15}^{\prime} B_{30}^{\prime}}{2 B_{21}^{\prime} \theta(1-\Gamma)}, \\
B_{34}^{\prime} & =\frac{1+\frac{B_{28}^{\prime}}{B_{21}^{\prime}}}{E}, \quad B_{35}^{\prime}=\frac{B_{29}^{\prime}}{B_{21}^{\prime} E}, \quad B_{36}^{\prime}=\frac{h^{-}+h^{+}-\frac{B_{30}^{\prime}}{B_{21}^{\prime}}}{E}, \\
F_{1}^{\prime} & =\frac{\left(1+2 E B_{32}^{\prime} B_{34}^{\prime}+2 E B_{31}^{\prime} B_{35}^{\prime}\right)^{2}}{\left(9 B_{34}^{\prime 2}\right)}, \quad F_{2}^{\prime}=\frac{2 E B_{32}^{\prime}-2 E B_{33}^{\prime} B_{34}^{\prime}+2 E B_{31}^{\prime} B_{36}^{\prime}}{27 B_{34}^{\prime 3}}, \\
F_{3}^{\prime} & =\frac{\left(1+2 E B_{32}^{\prime} B_{34}^{\prime}+2 E B_{31}^{\prime} B_{35}^{\prime}\right)^{3}}{F_{4}^{\prime}}=\frac{1}{6 B_{34}^{\prime 2}}\left(1+2 E B_{32}^{\prime} B_{34}^{\prime}+2 E B_{31}^{\prime} B_{35}^{\prime}\right)\left(2 E B_{32}^{\prime}-2 E B_{33}^{\prime} B_{35}^{\prime}+2 E B_{31}^{\prime} B_{36}^{\prime}\right), \\
F_{5}^{\prime} & =\left(2 E B_{33}^{\prime}-2 E B_{31}^{\prime} h^{-}\right) /\left(2 B_{34}^{\prime}\right), \quad F_{6}^{\prime}=\sqrt{-\left(F_{1}^{\prime}-F_{2}^{\prime}\right)^{3}+\left(-F_{3}^{\prime}+F_{4}^{\prime}+F_{5}^{\prime}\right)^{2}}, \\
F_{7}^{\prime} & =\left(F_{3}^{\prime}-F_{4}^{\prime}-F_{5}^{\prime}+F_{6}^{\prime}\right)^{1 / 3} .
\end{aligned}
$$

\section{Appendix G. Proof of Theorem 4.9. The Distributor's optimizations in CS-SR}

In this case, the Hessian matrix of the distributor's profit function shown in equation (4.33) is

$$
H(m, I)=\left[\begin{array}{cc}
2 S_{2}^{\prime}+2 S_{10}^{\prime} & S_{2}^{\prime}+S_{11}^{\prime}-\frac{S_{4}^{\prime}}{E} I \\
S_{2}^{\prime}-\frac{S_{4}^{\prime}}{E} I+S_{11}^{\prime} & -\frac{S_{4}^{\prime}}{E} m-\frac{S_{6}^{\prime}}{E}+\frac{3 S_{5}^{\prime}}{E} I+2 S_{6}^{\prime}
\end{array}\right]
$$

Then, the following conditions must be true:

$$
\begin{aligned}
& \left|H_{1}(m, I)\right|=2 S_{2}^{\prime}+2 S_{10}^{\prime}<0 \\
& \left|H_{2}(m, I)\right|=\left(\frac{3 S_{5}^{\prime} S_{17}^{\prime}}{E}+2 S_{15}^{\prime} S_{18}^{\prime}\right) I-S_{15}^{\prime} S_{17}^{\prime} m-S_{15}^{\prime 2} I^{2}+S_{13}^{\prime} S_{17}^{\prime}-S_{18}^{\prime 2}>0
\end{aligned}
$$


where constant parameters to are defined as

$$
\begin{aligned}
& S_{1}^{\prime}=-\beta_{1}-\frac{\beta_{1} B_{28}^{\prime}}{B_{21}^{\prime}}-\frac{\beta_{2} B_{25}^{\prime}}{B_{24}^{\prime}}-\frac{\beta_{3} B_{11}^{\prime} B_{28}^{\prime}}{B_{13}^{\prime} B_{21}^{\prime}}+\frac{\beta_{3} B_{12}^{\prime} B_{25}^{\prime}}{B_{13}^{\prime} B_{24}^{\prime}}-\frac{\beta_{4} B_{15}^{\prime} B_{28}^{\prime}}{2(1-\Gamma) \theta B_{21}^{\prime}}-\frac{\beta_{4} B_{16}^{\prime} B_{25}^{\prime}}{2 B_{24}^{\prime} \theta(1-\Gamma)}, \\
& S_{2}^{\prime}=\frac{\beta_{1} B_{29}^{\prime}}{B_{21}^{\prime}}-\frac{\beta_{2} B_{26}^{\prime}}{B_{24}^{\prime}}+\frac{\beta_{3} B_{11}^{\prime} B_{29}^{\prime}}{B_{13}^{\prime} B_{21}^{\prime}}+\frac{\beta_{3} B_{12}^{\prime} B_{26}^{\prime}}{B_{13}^{\prime} B_{24}^{\prime}}-\frac{\beta_{4} B_{15}^{\prime} B_{29}^{\prime}}{2 \theta(1-\Gamma) B_{24}^{\prime}}+1, \\
& S_{3}^{\prime}=\beta_{0}+\frac{\beta_{1} B_{30}^{\prime}}{B_{21}^{\prime}}-\frac{\beta_{2} B_{27}^{\prime}}{B_{24}^{\prime}}+\frac{\beta_{3} B_{11}^{\prime} B_{30}^{\prime}}{B_{13}^{\prime} B_{21}^{\prime}}+\frac{\beta_{3} B_{12}^{\prime} B_{27}^{\prime}}{B_{13}^{\prime} B_{24}^{\prime}}+\frac{\beta_{3} B_{14}^{\prime}}{B_{13}^{\prime}}-\frac{\beta_{4} B_{15}^{\prime} B_{30}^{\prime}}{2(1-\Gamma) \theta B_{21}^{\prime}}-\frac{\beta_{4} B_{16}^{\prime} B_{27}^{\prime}}{2 \theta(1-\Gamma) B_{24}^{\prime}}+\frac{\beta_{4} B_{17}^{\prime}}{2 \theta(1-\Gamma)}, \\
& S_{4}^{\prime}=1+\frac{B_{28}^{\prime}}{B_{21}^{\prime}}, \quad S_{5}^{\prime}=\frac{B_{29}^{\prime}}{B_{21}^{\prime}}, \quad S_{6}^{\prime}=h^{+}+h^{-}-\frac{B_{30}^{\prime}}{B_{21}^{\prime}}, \\
& S_{7}^{\prime}=\frac{B_{26}^{\prime} B_{27}^{\prime} \phi \Delta}{B_{24}^{\prime 2}}-\frac{B_{16}^{\prime} B_{17}^{\prime} B_{26}^{\prime} \Gamma}{2 B_{24}^{\prime} \theta(1-\Gamma)^{2}}+\frac{B_{16}^{\prime 2} B_{26}^{\prime} B_{27}^{\prime} \Gamma}{2 B_{24}^{\prime 2} \theta(1-\Gamma)^{2}}-\frac{B_{15}^{\prime} B_{17}^{\prime} B_{29} \Gamma}{2 B_{21}^{\prime 2} \theta(1-\Gamma)^{2}}+\frac{B_{15}^{\prime} B_{16}^{\prime} B_{27}^{\prime} B_{29}^{\prime} \Gamma}{2 B_{21}^{\prime} B_{24}^{\prime} \theta(1-\Gamma)^{2}} \\
& +\frac{B_{15}^{\prime} B_{16}^{\prime} B_{26}^{\prime} B_{30}^{\prime} \Gamma}{2 B_{21}^{\prime} B_{24}^{\prime} \theta(1-\Gamma)^{2}}+\frac{B_{15}^{\prime 2} B_{29}^{\prime} B_{30}^{\prime} \Gamma}{2 B_{21}^{\prime 2} \theta(1-\Gamma)^{2}} \\
& S_{8}^{\prime}=\frac{B_{25}^{\prime} B_{27}^{\prime} \phi \Delta}{B_{24}^{\prime 2}}-\frac{B_{16}^{\prime} B_{17}^{\prime} B_{25}^{\prime} \Gamma}{2 B_{24}^{\prime} \theta(1-\Gamma)^{2}}+\frac{B_{16}^{\prime 2} B_{25}^{\prime} B_{27}^{\prime} \Gamma}{2 B_{24}^{\prime 2} \theta(1-\Gamma)^{2}}+\frac{B_{15}^{\prime} B_{17}^{\prime} B_{28} \Gamma}{2 B_{21}^{\prime 2} \theta(1-\Gamma)^{2}}-\frac{B_{15}^{\prime} B_{16}^{\prime} B_{27}^{\prime} B_{28}^{\prime} \Gamma}{2 B_{21}^{\prime} B_{24}^{\prime} \theta(1-\Gamma)^{2}} \\
& +\frac{B_{15}^{\prime} B_{16}^{\prime} B_{25}^{\prime} B_{30}^{\prime} \Gamma}{2 B_{21}^{\prime} B_{24}^{\prime} \theta(1-\Gamma)^{2}}-\frac{B_{15}^{\prime 2} B_{28}^{\prime} B_{30}^{\prime} \Gamma}{2 B_{21}^{\prime 2} \theta(1-\Gamma)^{2}}, \\
& S_{9}^{\prime}=\frac{B_{26}^{\prime 2} \phi \Delta}{2 B_{24}^{\prime 2}}+\frac{B_{16}^{\prime 2} B_{26}^{\prime 2} \Gamma}{4 B_{24}^{\prime 2} \theta(1-\Gamma)^{2}}+\frac{B_{15}^{\prime} B_{16}^{\prime} B_{26}^{\prime} B_{29}^{\prime} \Gamma}{2 B_{21}^{\prime} B_{24}^{\prime} \theta(1-\Gamma)^{2}}+\frac{B_{15}^{\prime 2} B_{29}^{\prime 2} \Gamma}{4 B_{21}^{\prime 2} \theta(1-\Gamma)^{2}}, \\
& S_{10}^{\prime}=\frac{B_{25}^{\prime 2} \phi \Delta}{2 B_{24}^{\prime 2}}+\frac{B_{16}^{\prime 2} B_{25}^{\prime} \Gamma}{4 B_{24}^{\prime 2} \theta(1-\Gamma)^{2}}+\frac{B_{15}^{\prime} B_{16}^{\prime} B_{25}^{\prime} B_{28}^{\prime} \Gamma}{2 B_{21}^{\prime} B_{24}^{\prime} \theta(1-\Gamma)^{2}}+\frac{B_{15}^{\prime 2} B_{28}^{\prime 2} \Gamma}{4 B_{21}^{\prime 2} \theta(1-\Gamma)^{2}}, \\
& S_{11}^{\prime}=\frac{B_{25}^{\prime} B_{26}^{\prime} \phi \Delta}{B_{24}^{\prime 2}}+\frac{B_{16}^{\prime 2} B_{25}^{\prime} B_{26}^{\prime} \Gamma}{4 B_{24}^{\prime 2} \theta(1-\Gamma)^{2}}-\frac{B_{15}^{\prime} B_{16}^{\prime} B_{26}^{\prime} B_{28}^{\prime} \Gamma}{2 B_{21}^{\prime} B_{24}^{\prime} \theta(1-\Gamma)^{2}}+\frac{B_{15}^{\prime} B_{16}^{\prime} B_{25}^{\prime} B_{29}^{\prime} \Gamma}{2 B_{21}^{\prime} B_{24}^{\prime} \theta(1-\Gamma)^{2}}-\frac{B_{15}^{\prime 2} B_{28}^{\prime} B_{29}^{\prime} \Gamma}{2 B_{21}^{\prime 2} \theta(1-\Gamma)^{2}}, \\
& S_{12}=S_{2}^{\prime}+S_{11}^{\prime}, \quad S_{13}=2 S_{9}^{\prime}-\frac{S_{6}^{\prime}}{E}, \quad S_{14}=\frac{3 S_{5}^{\prime}}{2 E}, \quad S_{15}^{\prime}=\frac{S_{4}^{\prime}}{E}, \quad S_{16}=S_{7}^{\prime}+h^{-}, \quad S_{17}^{\prime}=2 S_{1}^{\prime}+2 S_{10}^{\prime}, \\
& S_{18}^{\prime}=S_{2}^{\prime}+S_{11}^{\prime} \text {. }
\end{aligned}
$$

Then, the proof of Theorem 4.8 is straightforward by taking the partial derivatives of equation (4.33) with respect to the distributor's decision variables, setting both equal to zero, and solving both equations simultaneously. In other words, we have

$$
\begin{aligned}
& \frac{\partial \prod_{\mathrm{R}}}{\partial I}=S_{2}^{\prime} m+h^{-}-\frac{S_{4}^{\prime}}{E} m I-\frac{S_{6}^{\prime}}{E} I+\frac{3 S_{5}^{\prime}}{2 E} I^{2}+S_{7}^{\prime}+2 S_{9}^{\prime} I+S_{11}^{\prime} m=0 \\
& \frac{\partial \prod_{\mathrm{R}}}{\partial m}=2\left(S_{1}^{\prime}+S_{10}^{\prime}\right) m+\left(S_{2}^{\prime}+S_{11}^{\prime}\right) I-\frac{S_{4}^{\prime}}{2 E} I^{2}+S_{3}^{\prime}+S_{8}^{\prime}=0 .
\end{aligned}
$$

Then, the proof is complete when the nonlinear equations (G.5) and (G.6) are solved, which the other constant parameters $S_{19}^{\prime}-G_{7}$ used in Theorem 4.8 are

$$
\begin{aligned}
S_{19}^{\prime} & =\frac{S_{4}^{\prime}}{2 E}, \quad S_{20}^{\prime}=S_{3}^{\prime}+S_{8}^{\prime}, \\
G_{1} & =\frac{\left(S_{14}^{\prime} S_{17}^{\prime}+S_{15}^{\prime} S_{18}^{\prime}+S_{12}^{\prime} S_{19}^{\prime}\right)^{2}}{\left(9 S_{15}^{\prime 2} S_{19}^{\prime 2}\right)}, \quad G_{2}=\frac{\left(S_{13}^{\prime} S_{17}^{\prime}-S_{12}^{\prime} S_{18}^{\prime}+S_{15}^{\prime} S_{20}^{\prime}\right)}{\left(3 S_{15}^{\prime} S_{19}^{\prime}\right)}, \quad G_{3}=\frac{\left(S_{14}^{\prime} S_{17}^{\prime}+S_{15}^{\prime} S_{18}^{\prime}+S_{12}^{\prime} S_{19}^{\prime}\right)^{3}}{27 S_{15}^{\prime 3} S_{19}^{\prime 3}}, \\
G_{4} & =\frac{-S_{16}^{\prime} S_{17}^{\prime}+S_{12}^{\prime} S_{20}^{\prime}}{2 S_{15}^{\prime} S_{19}^{\prime}}, \quad G_{5}=\frac{\left(\left(S_{14}^{\prime} S_{17}^{\prime}+S_{15}^{\prime} S_{18}^{\prime}+S_{12}^{\prime} S_{19}^{\prime}\right)\left(S_{13}^{\prime} S_{17}^{\prime}-S_{12}^{\prime} S_{18}^{\prime}+S_{15}^{\prime} S_{20}^{\prime}\right)\right)}{\left(6 S_{15}^{\prime 2} S_{19}^{\prime 2}\right)} \\
G_{6} & =\sqrt{\left(G_{1}+G_{2}\right)^{3}+\left(G_{3}-G_{4}+G_{5}\right)^{2}}, \quad G_{7}=\left(G_{3}-G_{4}+G_{5}+G_{6}\right)^{1 / 3}
\end{aligned}
$$


Acknowledgements. The authors are thankful for invaluable comments provided by the reviewers. Taking care of the comments improved the presentation of this paper significantly.

\section{REFERENCES}

[1] G.A. Albuquerque, P. Maciel and R.M.F. Lima, Strategic and tactical evaluation of conflicting environment and business goals in green supply chains. IEEE Trans. Syst. Man Cybern. Syst. 43 (2013) 1013-1027.

[2] A. Atasu, M. Sarvary and L.N. Van Wassenhove, Remanufacturing as a marketing strategy. Manage. Sci. 54 (2008) $1731-1746$.

[3] S. Benjaafar, Y. Li and M. Daskin, Carbon footprint and the management of supply chains: insights from simple models. IEEE Trans. Autom. Sci. Eng. 10 (2013) 99-116.

[4] M. Bhattacharyya and S.S. Sana, A mathematical model on eco-friendly manufacturing system under probabilistic demand. RAIRO: OR 53 (2019) 1899-1913.

[5] K. Bi, P. Huang and X. Wang, Innovation performance and influencing factors of low-carbon technological innovation under the global value chain: a case of Chinese manufacturing industry. Technol. Forecasting Soc. Change 111 (2016) 275-284.

[6] C.F. Böttcher and M. Müller, Drivers, practices and outcomes of low-carbon operations: approaches of German automotive suppliers to cutting carbon emissions. Bus. Strategy Environ. 24 (2015) 477-498.

[7] A. Boustani, S. Sahni, T. Gutowski and S. Graves, Remanufacturing and energy savings. Massachusetts Institute of Technology. Dept. of Mechanical Engineering. http://hdl.handle.net/1721.1/58461 (2010).

[8] E. Cao and M. Yu, Trade credit financing and coordination for an emission-dependent supply chain. Comput. Ind. Eng. 119 (2018) 50-62.

[9] Cambridge Enterprise, Battery paste recycling technology developed at Cambridge University [Accessed on 29 Jun 2016]. https://www.enterprise.cam.ac.uk/news/eco-friendly-battery-pasterecycling- technology-developed-universitycambridge-licensed-aurelius-environmental/(2016).

[10] G.H. Chao, S.M.R. Iravani and R.C. Savaskan, Quality improvement incentives and product recall cost sharing contracts. Manage. Sci. $\mathbf{5 5}$ (2009) 1122-1138.

[11] Y. Cheng and P. Zhang, Pricing strategy and coordination in an O2O Mixed channel supply chain with carbon cap-and-trade mechanism. In: Proceedings of the 36th Chinese Control Conference (CCC). Dalian, China (2017).

[12] T.M. Choi, Y. Li and L. Xu, Channel leadership, performance and coordination in closed loop supply chains. Int. J. Prod. Econ. 146 (2013) 371-380.

[13] CRR, Carbon footprints of tyre production - new versus remanufactured. http://www.remanufacturing.org.uk/pdf/story/ 1p158.pdf (2008).

[14] S.E. Daniel, C.P. Pappis and T.G. Voutsinas, Applying life cycle inventory to reverse supply chains: a case study of lead recovery from batteries. Res. Conserv. Recycl. 37 (2003) 251-281.

[15] C. Dong, B. Shen, P.-S. Chow, L. Yang and C.T. Ng, Sustainability investment under cap-and trade regulation. Ann. Oper. Res. 240 (2016) 509-531.

[16] D. Drake and S. Spinler, Sustainable operations management: an enduring stream or a passing fancy. Manuf. Serv. Oper. Manage. 15 (2013) 689-700.

[17] S. Du, F. Ma, Z. Fu, L. Zhu and J. Zhang, Game-theoretic analysis for an emission-dependent supply chain in a "cap-and-trade" system. Ann. Oper. Res. 228 (2011) 135-149.

[18] Y. Fernando, W.X. Wah and M.S. Shaharudin, Does a firm's innovation category matter in practicing eco-innovation? Evidence from the lens of Malaysia companies practicing green technology. J. Manuf. Technol. Manage. 27 (2016) $208-233$.

[19] G. Ferrer and J.M. Swaminathan, Managing new and differentiated remanufactured products. Eur. J. Oper. Res. 203 (2010) $370-379$.

[20] B. Fu, Z. Shu and X. Liu, Blockchain enhanced emission trading framework in fashion apparel manufacturing industry. Sustainability 10 (2018) 1105.

[21] J. Gao, H. Han, L. Hou and H. Wang, Pricing and effort decisions in a closed-loop supply chain under different channel power structures. J. Cleaner Prod. 112 (2016) 2043-2205.

[22] B.C. Giri, A. Chakraborty and T. Maiti, Quality and pricing decisions in a two-echelon supply chain under multi-manufacturer competition. Int. J. Adv. Manuf. Technol. 78 (2015) 1927-1941.

[23] B.C. Giri, C. Mondal and T. Maiti, Analysing a closed-loop supply chain with selling price, warranty period and green sensitive consumer demand under revenue sharing contract. J. Cleaner Prod. 190 (2018) 822-837.

[24] B.C. Giri, C. Mondal and T. Maiti, Optimal product quality and pricing strategy for a two-period closed-loop supply chain with retailer variable markup. RAIRO: OR 53 (2019) 609-626.

[25] V.D.R. Guide Jr. and L.N. Van Wassenhove, OR FORUM-the evolution of closed-loop supply chain research. Oper. Res. 57 (2009) 10-18.

[26] H.A. Hadi, H.H. Purba, K.S. Indarto, R.G.P. Simarmata, G.P. Putra, D. Ghazali and S. Aisyah, The implementation of quality function deployment (QFD) in tire industry. ComTech: Comput. Math. Eng. App. 8 (2017). DOI: $10.21512 /$ comtech.v8i4.3792.

[27] A. Hafezalkotob, Competition, cooperation, and coopetition of green supply chains under regulations on energy saving levels. Transp. Res. Part E 97 (2017) 228-250.

[28] A. Hafezalkotob, Direct and indirect intervention schemas of government in the competition between green and non-green supply chains. J. Cleaner Prod. 170 (2018) 753-772. 
[29] H\&M, The H\&M group sustainability report [Accessed on 4 April 2017]. http://sustainability.hm.com/content/dam/hm/ about/documents/en/CSR/Report\%202016/HM_group_SustainabilityReport_2016_FullReport_en.pdf (2016).

[30] Z. Hong and X. Guo, Green product supply chain contracts considering environmental responsibilities. Omega 83 (2019) $155-166$.

[31] X. Hong, L. Xu, P. Du and W. Wang, Joint advertising, pricing and collection decisions in a closed loop supply chain. Int. J. Prod. Econ. 167 (2015) 12-22.

[32] Y. Huang and Z. Wang, Closed-loop supply chain models with product take-back and hybrid remanufacturing under technology licensing. J. Cleaner Prod. 142 (2017) 3917-3927.

[33] M. Huang, M. Song, L.H. Lee and W.K. Ching, Analysis for strategy of closed-loop supply chain with dual recycling channel. Int. J. Prod. Econ. 144 (2013) 510-520.

[34] M.Y. Jaber, C.H. Glock and A.M.A. El Saadany, Supply chain coordination with emission reduction incentives. Int. J. Prod. Econ. 51 (2013) 69-82.

[35] T. Javadi, N. Alizadeh-basban, S. Asian and A. Hafezalkotob, Pricing policies in a dual-channel supply chain considering flexible return and energy-saving regulations. Comput. Ind. Eng. 135 (2019) 655-674.

[36] J. Ji, Z. Zhang and L. Yang, Carbon emission reduction decisions in the retail-/dual-channel supply chain with consumers' preference. J. Cleaner Prod. 141 (2017) 852-867.

[37] M. Jin, R. Tang, Y. Ji, F. Liu, L. Gao and D. Huisingh, Impact of advanced manufacturing on sustainability: an overview of the special volume on advanced manufacturing for sustainability and low fossil carbon emissions. J. Cleaner Prod. 161 (2017) 69-74.

[38] C. Jingxian, L. Liang, Y. Dong-Qing and S. Shengnan, Price and quality decisions in dual-channel supply chains. Eur. J. Oper. Res. 259 (2017) 935-948.

[39] V.R. Kannan and K.C. Tan, Just in time, total quality management, and supply chain management: understanding their linkages and impact on business performance. Omega 33 (2005) 153-162.

[40] M. Lashgari, A.A. Taleizadeh and A. Ahmadi, Partial up-stream advanced payment and partial down-stream delayed payment in a three-level supply chain. Ann. Oper. Res. 238 (2016) 329-354.

[41] M. Lashgari, A.A. Taleizadeh and S.S. Sana, An inventory control problem for deteriorating items with back-ordering and financial considerations under two levels of trade credit linked to order quantity. J. Ind. Manage. Optim. 12 (2016) $1091-1119$.

[42] Y. Li, L. Xu and D. Li, Examining relationships between the return policy, product quality and pricing strategy in online direct selling. Int. J. Prod. Econ. 144 (2013) 451-460.

[43] X. Li, D. Shi, Y. Li and X. Zhen, Impact of carbon regulations on the supply chain with carbon reduction effort. IEEE Trans. Syst. Man Cybern. Syst. 99 (2017) 1-10.

[44] J. Li, J. Lu, Q. Wang and C. Li, Quality and pricing decisions in a two-echelon supply chain with nash bargaining fairness concerns. Discrete Dyn. Nat. Soc. 3 (2018) 1-19.

[45] Z. Liu, T.D. Anderson and J.M. Cruz, Consumer environmental awareness and competition in two-stage supply chains. Eur. J. Oper. Res. 218 (2012) 602-613.

[46] Z. Luo, A. Gunasekaran, R. Dubey, S.J. Childe and T. Papadopoulos, Antecedents of low carbon emissions supply chains. Int. J. Clim. Change Strategies Manage. 9 (2017) 707-727.

[47] P. Ma, C. Zhang, X. Hong and H. Xu, Pricing decisions for substitutable products with green manufacturing in a competitive supply chain. J. Cleaner Prod. 183 (2018) 618-640.

[48] S.R. Madani and M. Rasti-Barzoki, Sustainable supply chain management with pricing, greening and governmental tariffs determining strategies: a game-theoretic approach. Comput. Ind. Eng. 105 (2017) 287-298.

[49] W. Moon, W.J. Florkowski, B. Brückner and I. Schonhof, Willingness to pay for environmental practices: implications for eco-labeling. Land Econ. 78 (2002) 88-102.

[50] S. Moshtagh and A.A. Taleizadeh, Stochastic integrated manufacturing and Remanufacturing model with Shortage, rework and quality based return rate in a closed loop supply chain. J. Cleaner Prod. 141 (2017) 1548-1573.

[51] MTD, Modern Tire Dealer, Modern Tire Dealer [Accessed on 22 Aug 2018]. http://www.moderntiredealer.com/.

[52] A. Nagurney and M. Yu, Sustainable fashion supply chain management under oligopolistic competition and brand differentiation. Int. J. Prod. Econ. 135 (2012) 532-540.

[53] A. Örsdemir, E. Kemahlığlu-Ziya and A.K. Parlaktürk, Competitive quality choice and remanufacturing. Prod. Oper. Manage. 23 (2014) 48-64.

[54] N. Pourmohammad Zia and A.A. Taleizadeh, A lot-sizing model with backordering under hybrid linked-to-order multiple advance payments and delayed payment. Transp. Res.: Part E 82 (2015) 19-37.

[55] J.A. Rodríguez, C. Giménez and D. Arenas, Cooperative initiatives with NGOs in socially sustainable supply chains: How is inter-organizational fit achieved? J. Cleaner Prod. 137 (2016) 516-526.

[56] A. Rong, R. Akkerman and M. Grunow, An optimization approach for managing fresh food quality throughout the supply chain. Int. J. Prod. Econ. 131 (2011) 421-429.

[57] R.C. Savaskan and L.N. Van Wassenhove, Reverse channel design: the case of competing retailers. Manage. Sci. 52 (2006) $1-14$.

[58] M. Sinayi and M. Rasti-Barzoki, A game theoretic approach for pricing, greening, and social welfare policies in a supply chain with government intervention. J. Cleaner Prod. 196 (2018) 1443-1458.

[59] S. Swami and J. Shah, Channel coordination in green supply chain management. J. Oper. Res. Soc. 64 (2013) $336-351$. 
[60] H. Song and X. Gao, Green supply chain game model and analysis under revenue-sharing contract. J. Cleaner Prod. 170 (2018) 183-192.

[61] G.C. Souza, Closed-loop supply chains: a critical review, and future research. Decis. Sci. 44 (2013) 7-38.

[62] A.A. Taleizadeh, Lot sizing model with advance payment and disruption in supply under planned partial backordering. Int. Trans. Oper. Res. 24 (2017) 783-800.

[63] A.A. Taleizadeh and M. Noori-Daryan, Pricing, replenishments and production policies in a supply chain of pharmacological product with rework process: a game theoretic approach. Oper. Res. Int. J. 16 (2016) 89-115.

[64] A.A. Taleizadeh and R. Sadeghi, Pricing strategies in the competitive reverse supply chains with traditional and E-channels: a game theoretic approach. Int. J. Prod. Econ. 215 (2019) 48-60.

[65] A.A. Taleizadeh, N. Alizadeh-Basban and S.T.A. Niaki, A closed-loop supply chain considering carbon reduction, quality improvement effort, and return policy under two remanufacturing scenarios. J. Cleaner Prod. 232 (2019) 1230-1250.

[66] A.A. Taleizadeh, N. Alizadeh-Basban and B.R. Sarker, Coordinated contracts in a two-echelon supply chain considering pricing strategy. Comput. Ind. Eng. 124 (2018) 249-275.

[67] A.A. Taleizadeh, S.G.H. Jalali-Naini, H.M. Wee and T.C. Kuo, An imperfect, multi product production system with rework. Sci. Iran. 20 (2013) 811-823.

[68] A.A. Taleizadeh, M.S. Moshtagh and I. Moon, Optimal decisions of price, quality, effort level, and return policy in a three-level closed-loop supply chain based on different game theory approaches. Eur. J. Ind. Eng. 11 (2017) 486-525.

[69] A.A. Taleizadeh, M.S. Moshtagh and I. Moon, Pricing, product quality, and collection optimization in a decentralized closedloop supply chain with different channel structures: Game theoretical approach. J. Cleaner Prod. 189 (2018) $406-431$.

[70] A.A. Taleizadeh, M. Noori-Daryan and K. Govindan, Pricing and ordering decisions of two competing supply chains with different composite policies: a Stackelberg game-theoretic approach. Int. J. Prod. Res. 54 (2016) 2807-2836.

[71] A.A. Taleizadeh, M. Perak Sari-khanbeglo and L.E. Cárdenas-Barrón, An EOQ inventory model with partial backordering and reparation of imperfect products. Int. J. Prod. Econ. 182 (2016) 418-434.

[72] W. Tong, D. Mu, F. Zhao, G.P. Mendis and W.J. Sutherland, The impact of cap-and-trade mechanism and consumers' environmental preferences on a retailer-led supply Chain. Res. Conserv. Recycl. 142 (2019) 88-100.

[73] M. Varsei, C. Soosay, B. Fahimnia and J. Sarkis, Framing sustainability performance of supply chains with multi-dimensional indicators. Int. J. Supply Chain Manage. 19 (2014) 242-257.

[74] Q. Wang, D. Zhao and L. He, Contracting emission reduction for supply chains considering market low-carbon preference. J. Cleaner Prod. 120 (2016) 72-84.

[75] L. Wang, L. Zhao, G. Mao, J. Zuo and H. Du, Way to accomplish low carbon development transformation: a bibliometric analysis during 1995-2014. In: Renewable and Sustainable Energy Reviews. Elsevier, New York, NY (2017).

[76] M. Wang, L. Zhao and M. Herty, Modelling carbon trading and refrigerated logistics services within a fresh food supply chain under carbon cap-and-trade regulation. Int. J. Prod. Res. 56 (2018) 4207-4225.

[77] C.H. Wu, Product-design and pricing strategies with remanufacturing. Eur. J. Oper. Res. 222 (2012) $204-215$.

[78] T.J. Xiao and D.Q. Yang, Risk sharing and information revelation mechanism of a one-manufacturer and one-retailer supply chain facing an integrated competitor. Eur. J. Oper. Res. 196 (2009) 1076-1085.

[79] G. Xie, S. Wang and K.K. Lai, Quality improvement in competing supply chains. Int. J. Prod. Econ. 134 (2011) 262-270.

[80] W. Xiong, Coordination and incentive in a supply chain with manufacture quality effort. In: 26th Chinese Control and Decision Conference. Zhejiang University, 2014 CCDC (2014).

[81] E.S. Zerang, A.A. Taleizadeh and J. Razmi, Analytical comparisons in a three-echelon closed-loop supply chain with price and marketing effort-dependent demand: game theory approaches. Environ. Dev. Sustainability 20 (2018) 451-478.

[82] B. Zhang and L. Xu, Multi-item production planning with carbon cap and trade mechanism. Int. J. Prod. Econ. 144 (2013) $118-127$.

[83] H. Zhang and D. Hong, Supplier's joint investments in cost reduction and quality improvement in a decentralized supply chain. Math. Prob. Eng. 2017 (2017) 4391475.

[84] J. Zhao, J. Wei and Y. Li, Pricing decisions for substitutable products in a two-echelon supply chain with firms' different channel powers. Int. J. Prod. Econ. 153 (2014) 243-252.

[85] Y. Zhou, F. Hu and Z. Zhou, Pricing decisions and social welfare in a supply chain with multiple competing retailers and carbon tax policy. J. Cleaner Prod. 190 (2018) 752-777.

[86] J. Zuckerman, K. Laughlin, D. Abramskiehn and X. Wang, Cap and trade in practice: barriers and opportunities for industrial emissions reductions in California. https://www.climatepolicyinitiative.org/ (2014). 